MEDIZINISCHE

UNIVERSITÄT

INNSBRUCK

\title{
P300 and CBP targeting in castration therapy resistant prostate cancer
}

Thesis for obtaining the degree of

Doctor of Philosophy (PhD)

Department of Urology

Division of Experimental Urology

Medical University Innsbruck

Submitted by

Tobias Furlan, MSc

Innsbruck, April 2021

PhD Program: Molecular and Cellular Biology of Diseases (MCBD) 


\section{EIDESSTATTLICHE ERKLÄRUNG}

Ich, Tobias Furlan , erkläre an Eides statt, dass ich die vorliegende Arbeit selbstständig verfasst, andere als die angegebenen Quellen/Hilfsmittel nicht benutzt und die den benutzten Quellen wörtlich oder inhaltlich entnommenen Stellen als solche kenntlich gemacht habe.

Diese Arbeit wurde bisher bei keiner Hochschule oder Universität zur Erlangung eines akademischen Abschlusses oder Diploms eingereicht.

\section{STATEMENT OF ORIGINALITY}

I, Tobias Furlan_, declare that I have authored this thesis independently, that I have not used other than the declared sources / resources and that I have explicitly marked all material which has been quoted either literally or by content from the used sources.

This work has not previously been submitted for a degree or diploma in any university. 


\section{Index}

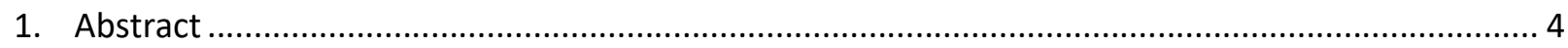

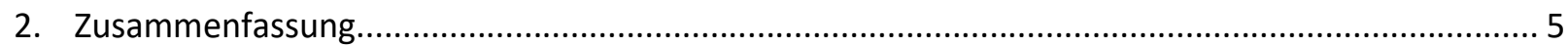

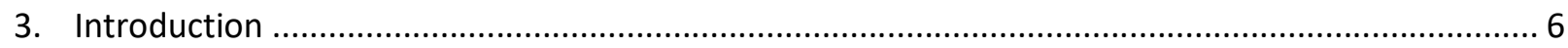

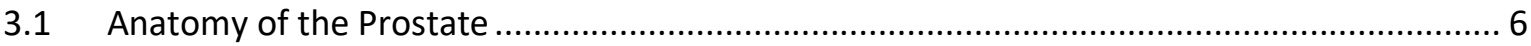

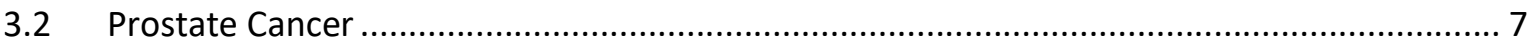

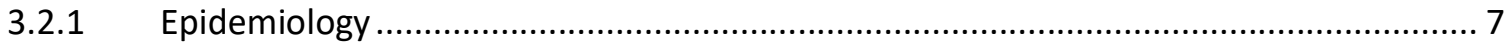

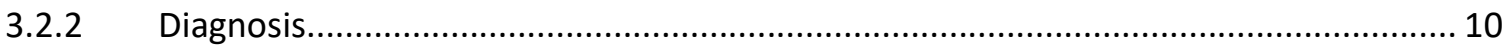

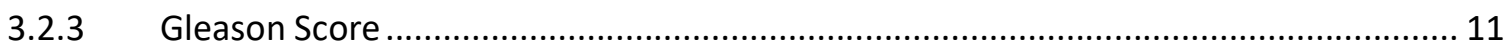

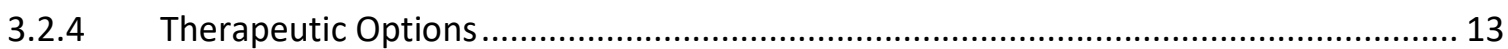

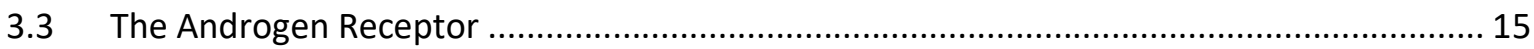

3.4 Enzalutamide and Androgen Deprivation Therapy Resistance ......................................... 17

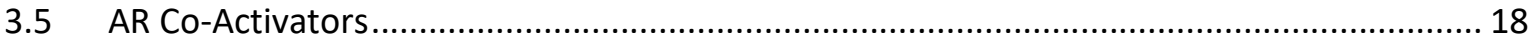

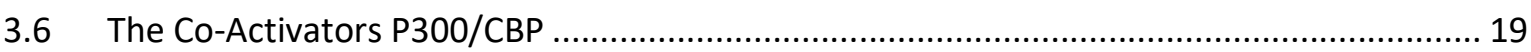

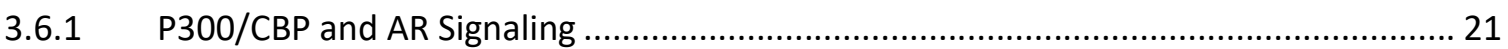

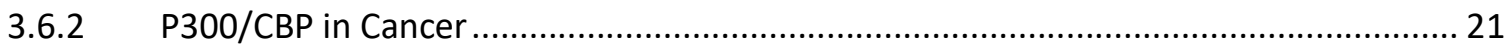

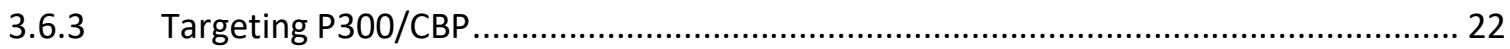

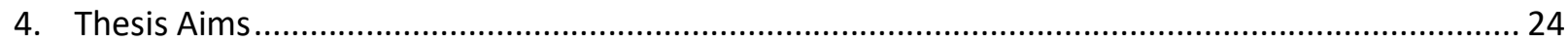

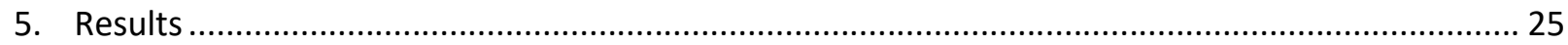

5.1 MYC-Mediated Ribosomal Gene Expression Sensitizes Enzalutamide-resistant Prostate

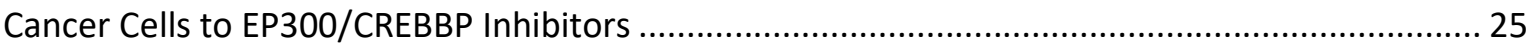

5.2 p300 is upregulated by docetaxel and is a target in chemoresistant prostate cancer ......... 26 


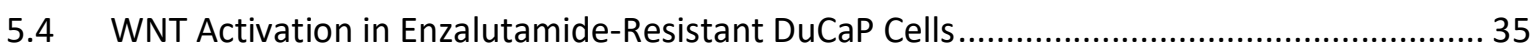

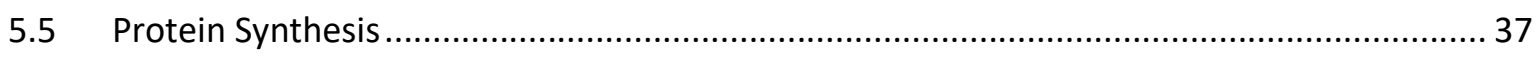

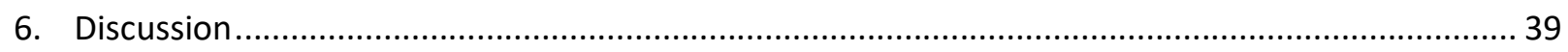

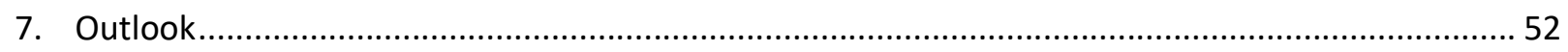

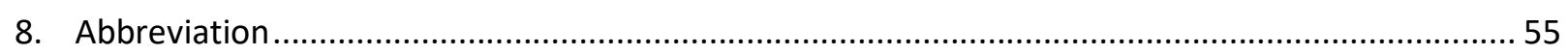

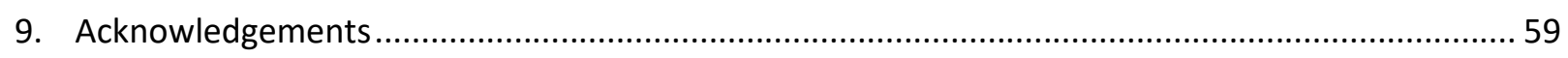

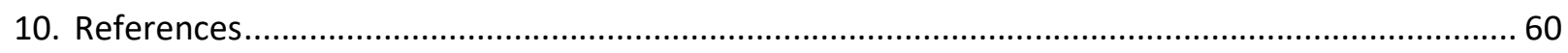

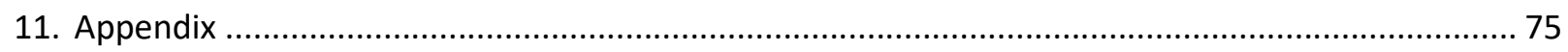




\section{Abstract}

For therapy of advanced prostate cancer second generation anti androgens such as enzalutamide are used. Although effective for a limited time period, those therapies are palliative. Various mechanisms have been reported to induce resistance after enzalutamide therapy. Generally, a reactivation of androgen receptor (AR) signaling could be observed. Previous studies demonstrated that the histone acetyltransferase P300 is highly expressed in prostate cancer and that targeting P300 via small molecular inhibitors reduced growth of prostate cancer cells. In the present study, we have characterized the effect of P300/CBP inhibitor treatment on enzalutamide-resistant prostate cancer. Viability and AR activity were determined for enzalutamide-resistant and parental cells after treatment with histone acetyltransferase (HAT) and bromodomain specific inhibitors of P300/CBP. The P300/CBP inhibitors efficiently reduced viability of all cell lines. Interestingly, we observed that the P300/CBP inhibitors were more effective in the enzalutamide-resistant cell lines in comparison to parental cells. Both inhibitors were able to down-regulate AR activity. However, there were no differences between enzalutamide-resistant and control cells. RNA-seq was performed to identify a subgroup of ribosomal proteins that are elevated in both enzalutamide-resistant sublines. Additionally, an overlapping subgroup of ribosomal proteins down-regulated upon inhibitor treatment was identified. Expression of ribosomal proteins correlates well with the signaling pathway of MYC, which was increasingly active in enzalutamide-resistant cell lines and down-regulated by P300/CBP inhibitors. Knockdown of MYC was sufficient to reduce expression of overexpressed ribosomal proteins. In summary, our results indicate that $\mathrm{P} 300 / \mathrm{CBP}$ and $\mathrm{MYC}$ are implicated in regulation of ribosomal proteins thus contributing to resistance to enzalutamide in prostate cancer. 


\section{Zusammenfassung}

Für die Therapie von fortgeschrittenen oder metastatischen Prostatakrebs kommen meist Androgen-Deprivations-Therapie oder Anti-androgene zum Einsatz. Jedoch entwickelt der Tumor in den meisten Fällen rasch eine Resistenz. Deshalb ist es wichtig neue therapeutische Ziele zu erforschen. Zwei potentielle Kandidaten sind die nah verwandten Proteine P300 und CBP. Beide sind Co-Aktivatoren des Androgenrezeptors, interagieren aber auch mit vielen anderen Transkriptionsfaktoren und Signalwegen. Vorherige Studien konnten zeigen, dass pharmakologische Inhibition von P300 und CBP wirksam in Prostatakrebs Zell Linien ist. Ziel dieser Arbeit ist es die molekularen Auswirkungen von P300 und CBP Inhibitoren auf Enzalutamid-resistente Prostatakrebs Zelllinien zu charakterisieren. Behandlung mit zwei verschieden P300 und CBP Inhibitoren zeigt in einem Viabilitäts-Assay, dass Enzalutamid-resistente Zelllinien anfälliger als Parentale Zellen sind. Auch die Aktivität des Androgenrezeptors konnte durch die Behandlung mit P300 und CBP Inhibitoren reduziert werden. Die Reduktion der Aktivität des Androgenrezeptors korreliert jedoch nicht mit der höheren Effizienz der P300 und CBP Inhibitoren in Enzalutamidresistenten Zelllinien. RNA-Seq zeigte, dass die Expression mehrerer Gene die für ribosomale Proteine kodieren durch die P300 und CBP Inhibitoren runter reguliert wurden. Gene Set Enrichment Analysis zeigt zudem, dass Ziele des MYC Signalwegs, welcher als Masterregulator für Ribosomen gilt, auch runter reguliert wurde. Ergänzend konnten wir zeigen, dass Enzalutamid-resistente Zelllinien Genen für ribosomale Proteine und Ziele des MYC Signalwegs über-exprimieren. Zusammengefasst zeigen unsere Ergebnisse, dass Inhibitoren von P300 und CBP effizient in Modellen für fortgeschrittenen Prostatakrebs sind und, dass sich ihre Aktivität nicht auf den Androgenrezeptor-Signalweg beschränkt. 


\section{Introduction}

\subsection{Anatomy of the Prostate}

The human prostate is a small, typically, walnut-sized gland of the male reproductive system, which is located below the urinary bladder, around the urethra. It contains the entrance of the two ejaculatory ducts to the urethra, which are the unifications of the vas deferens and ducts of the seminal vesicles. The prostate originates from five different lobi, however only three zones are histologically distinguishable in the adult prostate: the transitional zone, the central zone and the peripheral zone. The transitional zone wraps around the urethra below the bladder and extends to the joining of urethra and ejaculatory ducts. This region is the smallest, representing approximately $5 \%$ of the volume of the whole prostate. Despite its small size, it is the prime location for benign prostatic hypertrophy $(\mathrm{BPH})$, which is commonly observed in elderly men. The central zone wraps around the ejaculatory ducts and is rarely associated with malignancies. The peripheral zone wraps around the urethra below the joining of ejaculatory ducts and cups the other zones, thereby making up approximately $70 \%$ of the prostate. The peripheral zone most commonly contains prostate cancer, but not BPH. The peripheral zone is covered ventrally by a nonglandular fibromuscular zone and a fibrous layer encapsulates the whole prostate $(1,2)$.

The principal function of the prostate is to supply fluid to the semen to support flow, metabolism and survival of the sperm cells. This fluid is excreted from tubular and alveolar glands of the prostate into the urethra. Single glands are lined with basal cells on which columnar luminal cells extend into the lumen. Of those, only the luminal cells have a secretory function and express the $A R$ at a high level. Although there are reports of 
intermediate cells expressing markers of both basal and luminal cells, it is not clear if they represent a unique cell type. Both basal and luminal cells largely derive from respective progenitor cells. Pluripotency of those progenitors also allows them to give rise to the other cell type under certain conditions, like tissue regeneration. Furthermore, it was shown that basal progenitor cells are able to differentiate into neuroendocrine cells (3), which are found in low number in the glands and surrounding tissue. The surrounding tissue engulfs the glands and consists predominantly of smooth muscle cells, which support secretion by contracting and fibroblasts, which modulate extracellular matrix (4).

\subsection{Prostate Cancer}

The prostate is highly prone to cancerous malignancies as compared to other tissues. Due to the high content of glandular structures, $99 \%$ of those cancers are adenocarcinomas deriving from the pre-carcinoma stages called atypical small acinar proliferation (ASAP) and high-grade prostatic intraepithelial neoplasia (PIN). Historically, luminal cells have been identified as sources for prostate cancer, based on markers expressed (5). However, more recent research suggests that also basal cell are able to form prostate cancer and that luminal markers found in prostate cancer do not necessarily correlate with progenitor cell markers (6).

\subsubsection{Epidemiology}

In males, prostate cancer is the second most frequent type of cancer by incidence, only preceded by lung cancer, and fifth by mortality (Figure ). In Europe, the disparity of incidence is high. The top three countries, Ireland, Estonia and Sweden show three times 
higher incidence as compared to the bottom three, which are Moldavia, Albania and Bosnia (Figure 3.1). Incidence does not correlate with mortality, suggesting that results on incidence might be skewed due to differences in testing. Mortality of prostate cancer is low in Mediterranean countries like Italy, Greece, Spain, Malta and France, while it is relatively high in Baltic countries. Previous studies attributed differences to nutrition, but definitive evidence is missing (7).

The most significant risk factor for prostate cancer is age. The incidence rises dramatically in males over the age of 55 years (Prostate Cancer - Cancer Stat Facts). Other known risk factors for prostate cancer are genetic dispositions and lifestyle. Especially ethnic background strongly influences both incidence and mortality. Previous studies could show that men of African or Caribbean descent have a higher incidence. Men from Asian descent have a lower incidence and mortality as compared to the white men (8). Genetic predisposition for prostate cancer are further confirmed in men with relatives who have been diagnosed with prostate cancer (9). 
Estimated age-standardized incidence and mortality rates (World) in 2020, worldwide, males, all ages

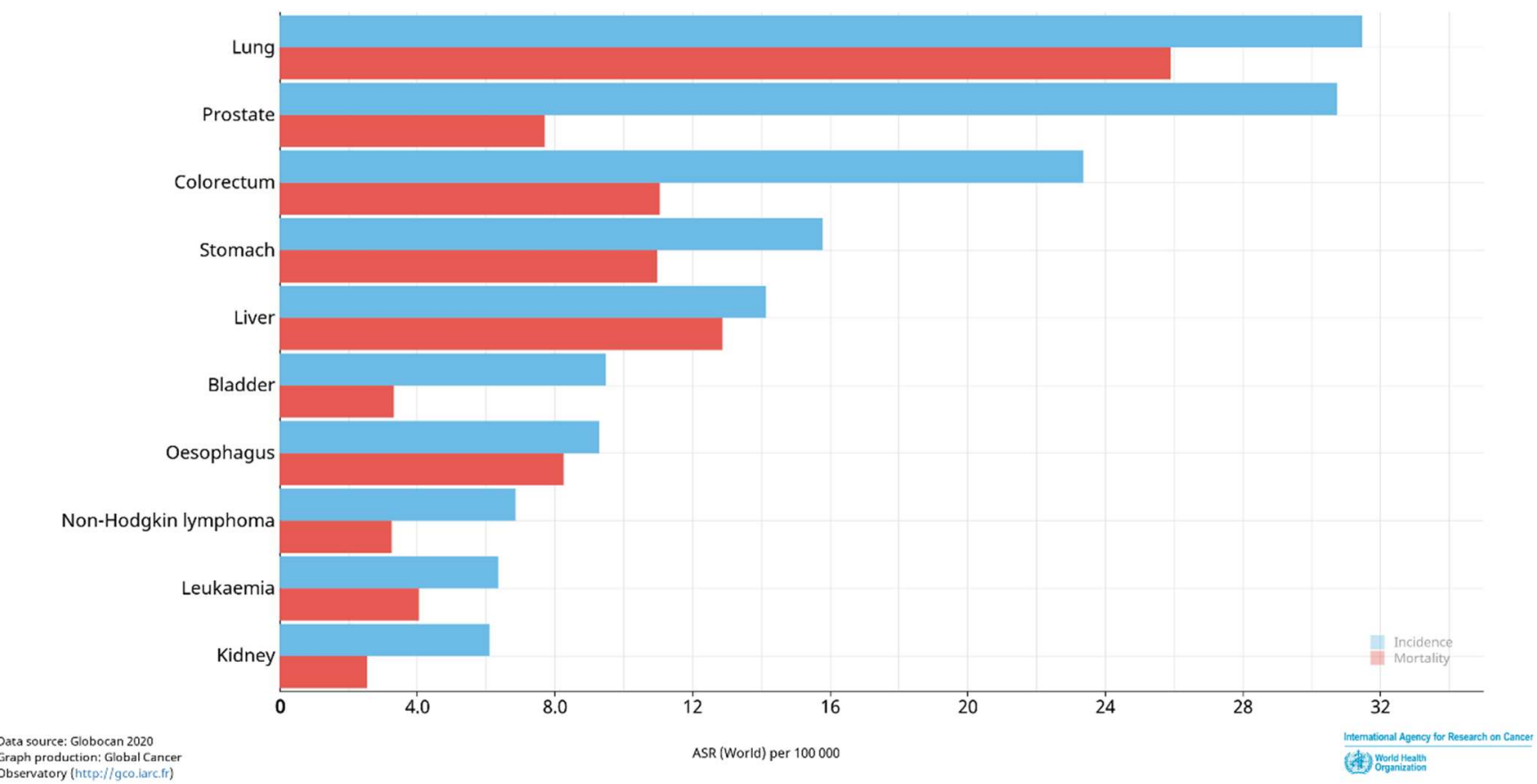

Figure 3.1: Estimated age-standardized incidence and mortality of the 10 most common cancer by site in males (10).

Estimated age-standardized incidence and mortality rates (World) in 2020, prostate, males, all ages

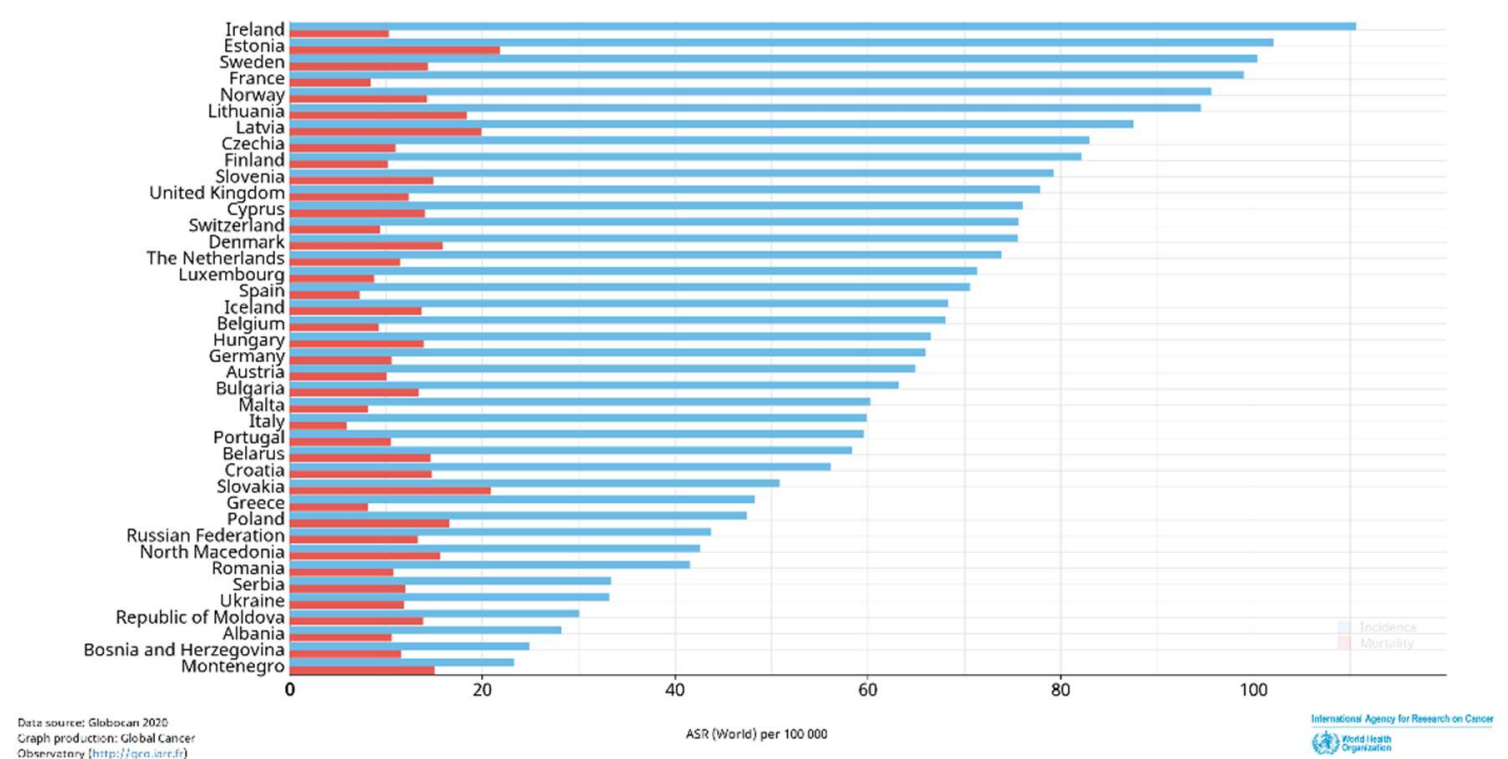

Figure 3.1: Estimated age-standardized incidence and mortality of prostate cancer of all European countries (10). 


\subsubsection{Diagnosis}

There are several non-invasive tests available to diagnose prostate cancer. Reasons to employ those can be symptoms like urinary or sexual problems, but since age is a risk factor, routine screening for prostate cancer is performed in older men. Digital rectal exam is performed to determine if there are hardened lumps in the prostate and how far they spread. Commonly, PSA levels in the blood are determined. Most of the PSA secreted from the prostate is a part of the seminal fluid, however a small part is secreted into the blood stream. Increased levels of PSA in the blood are a reliable marker for prostate cancer and correlate with progression. Serum PSA levels also rise with age. Therefore, an agedependent range of normal serum PSA levels was determined (Table 3.1). However, PSA screening for all men is controversial, as it might not reduce mortality while still burdening patients with stress and unnecessary treatments. Another problem with the before mentioned less invasive diagnostic tests is that tumor grade cannot be determined. Therefore, a needle biopsy of the prostate is performed, if there are suspicions of prostate cancer. This test is considerable more invasive, however it allows grading of the tumor.

Table 3.1: Normal range of PSA concentrations with the 95th percentile defined as upper limit. PSA rang was determined by measuring serum PSA levels of 471 randomly chosen western men without any signs for prostate cancer. Adapted from Oesterling et al. (11).

\begin{tabular}{|l|l|l|l|l|}
\hline Age & $40-49$ & $50-59$ & $60-69$ & $70-79$ \\
\hline Serum PSA concentrations $[\mathrm{ng} / \mathrm{ml}]$ & $0.0-2.5$ & $0.0-3.5$ & $0.0-4.5$ & $0.0-6.5$ \\
\hline
\end{tabular}




\subsubsection{Gleason Score}

Once prostate cancer has been diagnosed, it is usually graded to determine the progression and invasion capacity and to choose appropriate therapeutic strategies. The metastatic potential of prostate cancer is variable, in many cases prostate cancer growth is slow. Scoring of tumors is especially important to prevent overtreatment, as slowly growing prostate cancer do not necessarily require treatment.

Historically, grading of prostate cancer is done via Gleason score, which was introduced in 1966 (12). Since then several improvements to diagnosis have been made. With the discovery of blood PSA as a marker, prostate cancer could be diagnosed much earlier. In addition, immune staining for tissue slices was developed. Therefore, some adaptions to the grading system have been made over the years $(13,14)$. Nevertheless, the basis of the Gleason staging remained the same. For the Gleason score determination needle biopsies or tissue from radical prostatectomy (RP) are examined at a low magnification to determine growth pattern of cancerous tissue. Five different Gleason patterns are defined and a value between two and five is assigned (Figure 3.2) (15). 


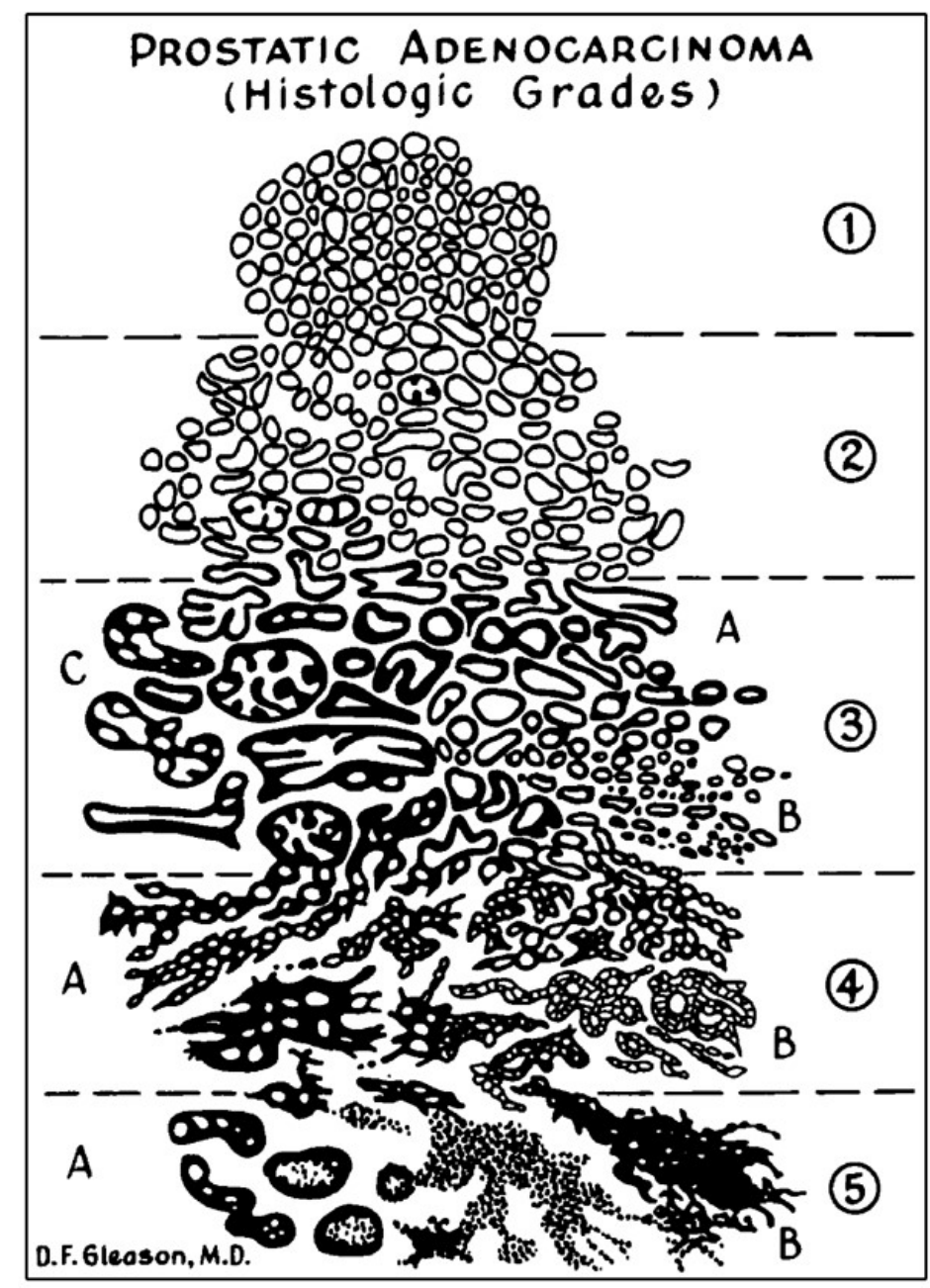

Figure 3.2: Sketch by Donald F. Gleason displaying Gleason patterns as well as different subtypes of Gleason patterns (16).

Gleason Pattern 1: The acini are round, of uniform morphology, tightly packed but well separated with no invasion of surrounding stroma. This pattern is very rare.

Gleason Pattern 2: The acini are still well defined but not round and of equal size anymore. The acini are more separated and there is limited invasion of the surrounding stroma. This pattern is most commonly found mixed with Gleason pattern 3.

Gleason Pattern 3: This pattern has three different forms. Pattern A consists of irregularly shaped acini, which extend into the stroma. Pattern B is similar but the acini are much 
smaller. Pattern $\mathrm{C}$ additionally displays a cribriform morphology. Pattern 3 is the most commonly diagnosed pattern and is considered a tumor of intermediate grade.

Gleason Pattern 4: The acini are undifferentiated and fused together with ragged borders. This pattern is considered high grade and is commonly found together with Gleason pattern 3.

Gleason Pattern 5: This pattern shows no glandular structure anymore, instead the cells form a sheet with ragged boarders. Necrotic cells are not uncommon. This pattern is also often found together with pattern 3.

To calculate Gleason score the value of the two most abundant patterns are added, thereby accounting for heterogeneity. If there is a third pattern of high grade but low volume it is also considered for diagnosis. A Gleason score of $1+1=2$ is nowadays not diagnosed anymore since it most likely represents only hyperplasia. Scores of up to 6 are still considered low risk as those cancers grow slowly and rarely invade surrounding tissue. Gleason score 7 represented by $3+4$ or $4+3$ are separated since $4+3$ is considered more aggressive. Scores between 8 and 10 are considered high risk cancer.

\subsubsection{Therapeutic Options}

The first line therapy is chosen dependent on tumor stage, Gleason score, PSA levels and patient status. The active surveillance strategy is often used for patients with Gleason score 6 or lower tumors which have not spread and have moderate PSA level increase. The aim of active surveillance is to prevent overtreatment by delaying therapy until it becomes 
necessary. For progressed prostate cancers which have not spread to distant organs localized therapy is used. Most frequently, the approaches are radical prostatectomy and radiation therapy. Prostate cancer, which has spread to distant locations, is currently not curable but well treatable, commonly with systemic approaches like hormone therapy or chemotherapy. Both hormone therapy and chemotherapy may be used together or in addition to radiation and surgery (17).

The AR is an important transcription factor for prostate cancer. The AR is activated by androgens and acts as a transcription factor for many genes involved in cell proliferation. Targeting the AR axis has proven to be effective for most prostate cancers (18). There are many ways to disrupt AR signaling using androgen deprivation therapy (ADT); historically, this was done via orchiectomy, which is the removal of the testicles (19). Although removal of the testicles drastically reduces androgen level residual levels of androgens are produced in the adrenal glands. Therefore, other therapies like luteinizing hormone release hormone (LHRH) agonists and antagonists, which induce reversible androgen deprivation, are more commonly used nowadays. Both LHRH agonists and antagonists reduce testosterone production of the testicles significantly, either via a negative feedback or via directly antagonizing luteinizing hormone action (LH). LHRH agonists and antagonists have been proven to be as effective as orchiectomy (20). Although ADT slows cancer growth and improves patients well-being, it is only effective for a limited period of time, after which the tumor is termed castration resistant prostate cancer (CRPC). Most common options for CRPC are anti androgen therapy and chemotherapy. CRPC is often characterized by reactivation of AR. Anti-androgen therapy is mostly still effective in CRPC, but its efficiency is similarly timely limited. 


\subsection{The Androgen Receptor}

The AR is a ligand dependent nuclear receptor belonging to the family of steroid receptors (21). Next to the AR the estrogen-, glucocorticoid-, mineralocorticoid- and progesterone receptor are a part of the steroid receptor family. Structurally, the AR and other steroid receptors contain an $\mathrm{N}$-terminal domain (NTD), DNA binding domain (DBD) and ligand binding domain (LBD) (Figure 3.4). As the name suggests the DBD is responsible for binding to the DNA while the LBD and NTD interact with transcription factors. The NTD is considered constitutively active while the LBD requires a ligand. The ligands of the AR are androgens, which are the male sex hormones. The principal androgen is testosterone, which is primarily synthesized and secreted by Leydig cells in the testis. Testosterone is released together with other androgen precursors, which can then be further processed to more active hormones by androgen dependent tissue. The most potent form of androgen is $5 \alpha-$ Dihydrotestosterone (DHT) (22). Upon binding of the AR by androgens the AR undergoes conformational changes, homodimerization and translocates to the nucleus, before binding to the DNA $(23,24)$. The binding sites of the AR are called androgen response elements (ARE) and are located at the promoters of AR target genes. AREs partially overlap within the steroid receptor family (25). Once bound to the ARE a complex of many different proteins is formed, containing scaffolds and co-activator proteins. The AR complex can then interact with the polymerase and thereby regulate transcription of specific genes. AR-specific transcription drives growth, development, maturation and maintenance of male reproductive organs and is also involved in many other processes. Although androgenmediated AR action is primarily associated with male physiology, previous work also implied a function in the regulation of muscle and bone growth in women (26). Furthermore, it has 
been shown that AR activity interferes with the function of the cardiovascular system, the immune system and other organs (27).

The $A R$ is an oncogenic driver in most prostate cancers and is therefore especially important. Growth of prostate cancer cells depends on AR signaling (28).

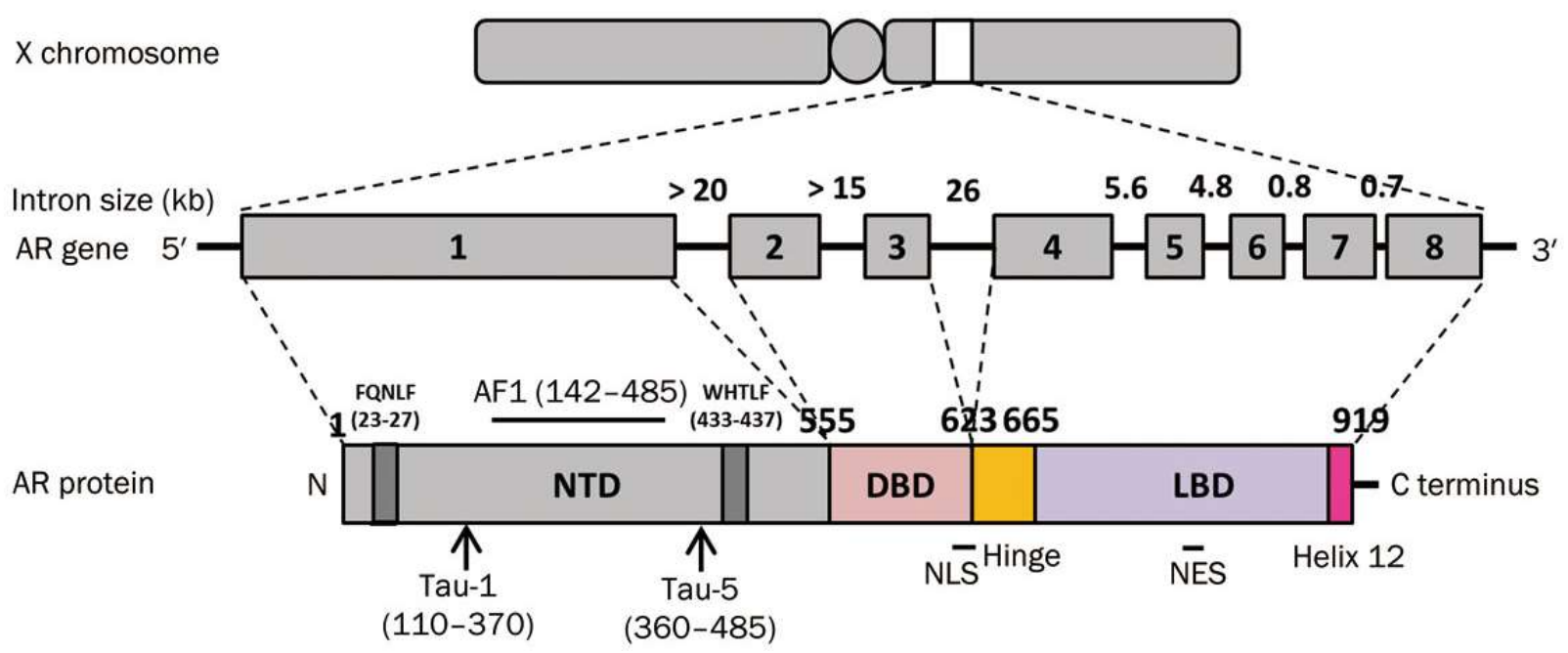

Figure 3.3: Location, architecture and domains of the AR. N-terminal domain (NTD). DNA-binding domain (DBD). Ligand-binding domain (LBD). FQNLF, WHTLF and activation function 1 (AF1) are motifs which bind to the ligand binding domain and stabilize bound androgen. The two transcription activation units (Tau-1 and Tau-2) partially overlap with AF1 and are transactivation regions. The ligand-binding domain (LBD) binds androgens and acts as a switch for AR activity. The Hinge domain is a flexible linker connecting DBD and LBD. The Hinge domain is not well-conserved but contains a nuclear localization signal (NLS). The counterpart to the NLS is the nuclear export signal (NES) in the LBD. Picture adapted from (29). 


\subsection{Enzalutamide and Androgen Deprivation Therapy Resistance}

ADT is a commonly used therapy in prostate cancer. However, development of resistance is inevitable. Some mechanisms behind ADT resistance are known. Most commonly AR signaling is reactivated, which can be achieved via different ways. The AR is often sensitized to low levels of androgens, either via increased expression of the AR (30) or mutations. Ligand-independent activation of the AR is also possible, for example via increased phosphorylation (31) or truncations of the AR. Best described is the AR variant 7 (32). The AR-V7 mutation establishes a cryptic exon 3 , which leads to an earlier termination of AR mRNA transcription and thereby an AR variant with missing ligand-binding domain. AR-V7 localizes to the nucleus without ligand binding and is constitutively active, but full length AR is still required as AR-V7 heterodimerizes with it (33). Synthesis of androgens from cholesterol directly in the prostate cancer tissue has also been described as a resistance mechanism (34). Finally, a resistance to ADT could be manifested by bypassing AR signaling, for example by activation of other steroid receptors (35) or up-regulation of anti-apoptotic proteins like BCL2 (36). Consequently, CRPC remains sensitive toward anti-androgens, which target the AR directly and which are therefore valuable in therapy.

Nowadays, the androgen inhibitor enzalutamide is routinely used for CRPC and metastatic CRPC (37). Enzalutamide is an orally administered non-steroidal anti-androgen. Enzalutamide directly binds to the AR, thereby preventing activation by $\mathrm{DHT}$, translocation into the nucleus and binding to DNA. Enzalutamide has been shown to cross the blood brain barrier, which is important for the treatment of brain metastases (38). Therapy with enzalutamide significantly reduces AR signaling and thereby prolongs progression-free survival. Initially, enzalutamide was used in patients who exhausted Docetaxel as treatment 
option, but newer clinical studies also show longer survival in patients who did not undergo chemotherapy (39). However, similar as with ADT a resistance develops inevitably $(40,41)$. Amplifications and overexpression of AR are commonly observed in enzalutamide-resistant prostate cancer. Increased amounts of AR can not only circumvent antagonist effect, but also turn the antagonist into a week agonist (42). Similarly, a mutation of the AR (F876L) has been shown to convert enzalutamide into an agonist (43). Therapeutic options following enzalutamide resistance, such as chemotherapy, are limited by severe side effects. Thus, there are no curative options for therapy resistant prostate cancer.

\subsection{AR Co-Activators}

The AR forms an intricate receptor complex, as previously mentioned. The composition, architecture and dynamics of this complex are still not entirely clear. After binding of the AR to DNA, co-factors of the p160 family are recruited. The p160 cofactors are mainly described as scaffold proteins that recruit histone-, methyltransferases, and polymerases to the complex of steroid receptors (44). Coactivators of the p160 family, especially SRC3, are often overexpressed in different types of cancer, mainly in breast and prostate cancer (45). Little is known about the exact mechanism of action of p160 proteins in cancer. Despite overexpression in hormone-dependent cancers there is also evidence pointing to pathways not mediated by hormones, like RAS (46) among others. Other prominent coactivators of the AR are Stat3 and PIAS. Stat3 is often overexpressed in prostate cancer and promotes migration and proliferation (47). PIAS, which was first discovered as an inhibitor of Stat3 also promotes proliferation via down-regulation of p21 (48). 


\subsection{The Co-Activators P300/CBP}

The two highly homologous proteins P300 (also called KAT3B or EP300) and CBP (also called KAT3A or CREBBP) are also a part of the AR receptor complex (Figure 3.4). P300 has initially been discovered as a transcriptional adaptor protein associated with E1A, a viral oncogene $(49,50)$. CBP, on the other hand, was first described as an adaptor to the CAMPregulated enhancer binding protein; as such, it is involved cAMP signaling. Nowadays it is known that both P300 and CBP are multifunctional co-factors of many transcription regulators and steroid receptors (51) as well as histone acetyl transferases for chromatin remodeling (52). It has been shown that P300 and CBP are required for development (53) and, dependent on the tissue, may act as tumor suppressors (54) or oncogenes (55). P300 and CBP can substitute for each other, due to their homology, however also distinctive functions are known (56). 

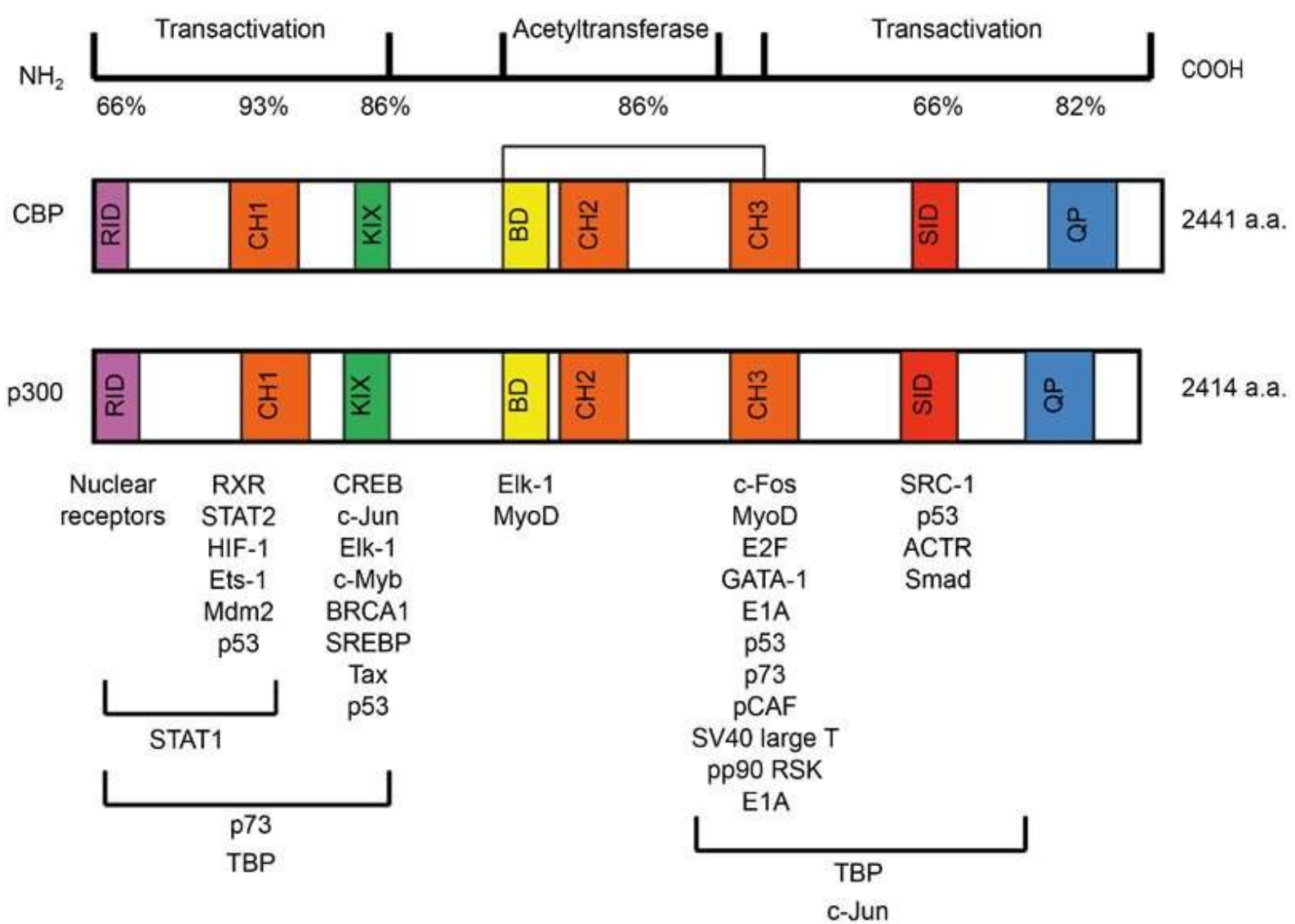

Figure 3.4: The two highly homologous coactivators P300 and CBP contain several other domains besides a bromodomain and a HAT: A nuclear receptor interaction domain (RID). Three cysteine/histidine rich regions ( $\mathrm{CH} 1, \mathrm{CH} 2$ and $\mathrm{CH} 3)$, which can be bound by zinc finger motifs of many different transcription factors. Interestingly different groups of transcription factors are bound by the different $\mathrm{CH}$ domains despite high homology. In addition, a kinase inducible domain (KIX) can also bind to different transcription factors. A steroid interaction domain (SID) and finally also a glutamine and proline rich domain (QP). Adapted from (57). 


\subsubsection{P300/CBP and AR Signaling}

P300 and CBP are important for AR signaling, as they are a crucial part of the AR receptor complex. They are generally recruited to the steroid receptor complexes by $\mathrm{p} 160$ cofactors, which bind to the steroid interaction domain (SID). Experimental evidence for the AR complex suggests that P300 directly binds to the AR NTD and SRC3 stabilizes this interaction, which is in contrast to other steroid receptors (58). P300 and CBP themselves then act as a scaffold to allow binding of other proteins. P300 and CBP can also interact with the polymerase II, thereby promoting transcription (59). Two highly conserved domains of P300 and CBP are a bromodomain, which is binds to acetylated proteins and a histone acetyl transferase domain (HAT). The HAT acetylates histones, thereby acting as an epigenetic regulator (60), as well as other proteins. It has been shown that acetylation of the AR by P300 and CBP is needed for full activation of AR signaling upon ligand binding (61). Not only acetylation of the AR is needed for efficient signaling but also histone acetylation, which relaxes the chromatin and allows binding of transcription factors (62). Together the bromodomain and the HAT form a positive feedback loop, as binding to an acetylated protein results in more acetylation.

\subsubsection{P300/CBP in Cancer}

P300 and CBP are involved in interaction with both oncogenes and tumor suppressors. The MYB oncogene for example, which commonly drives leukemia, requires P300. On the other hand, P300 and CBP are also involved in the acetylation, binding and activation of tumor suppressors such as p53 (63). P300 and CBP are also involved in hormonal cancers like breast cancer and prostate cancer due to their interaction with the steroid receptors (64). 
Previous data shows that P300 and CBP mRNA expression is increased in high grade and castration resistant prostate cancer and correlates with progression. However, this could not be confirmed on protein levels (65). P300 and CBP have been implicated in androgenindependent activation of the AR. Additionally, it has been shown that P300 expression is increased upon androgen deprivation $(66,67)$.

\subsubsection{Targeting P300/CBP}

As the importance of $\mathrm{P} 300$ and CBP in prostate cancer became more apparent and with the development and improvement of small molecular inhibitors therapeutic targeting of P300 and CBP became possible. The first compounds developed loosely targeted the bromodomain, which is conserved not only in P300 and CBP but also in many members of bromodomain and extra-terminal motif (BET) proteins. One of the first compounds, JQ1, is therefore not specific for P300 and CBP but also targets BRD2, BRD3 and BRD4. JQ1 was described as inhibitor of NUT midline carcinoma, which is driven by BRD3 or BRD4 fusion proteins (68). It was also discovered that JQ1 inhibits MYC-related signaling (69), in leukemia (70) and viral replication (71). One limiting factor of early BET inhibitors is their toxicity, which changed in newer generation of BET inhibitors and with the development of P300 and CBP specific inhibitors. Today several different inhibitors targeting either the HAT or bromodomain of P300, CBP and a variable range of BRD proteins exist. Several of those inhibitors showed promising results in prostate cancer, including: NEO2734, a dual BET and P300/CBP bromodomain inhibitor (72), C646 (73) and A-485 (74), two HAT inhibitors of P300 and CBP, I-CBP112 (75) and CCS1477 (65), two P300/CBP bromodomain inhibitors, and ZEN-3694 (76), a pan BET bromodomain inhibitor. Out of those CCS1477 will complete 
phase I/Ila trials in 2021 (NCT03568656) and ZEN-3694 will complete phase II trials in 2025 (NCT04471974). 


\section{Thesis Aims}

Enzalutamide treatment is currently used in prostate cancer therapy for ADT resistant prostate cancer, however resistance to enzalutamide is inevitable. Therefore, new therapeutic targets should be studied. P300 and CBP have many functions, among those they are co-activators of the AR. AR signaling is considered to be the main driver of prostate cancer and expression of P300 and CBP has been associated with prostate cancer. P300 and $\mathrm{CBP}$ are also involved in therapy resistance mechanisms, associated with reactivation of $A R$ signaling. Increased expression of P300 and CBP has been observed under androgen deprivation. With the development of small molecular inhibitors it became possible to target P300 and CBP pharmaceutically. Previous data could show that inhibition of P300 and CBP is effective in prostate cancer. Based on those results, we hypothesized that inhibition of P300 and CBP is also effective in enzalutamide-resistant prostate cancer. Therefore, we wanted to determine the potential of P300 and CBP inhibitors to reduce viability of different enzalutamide-resistant and -sensitive cell lines. In addition, we are also interested in the effects of P300 and CBP inhibition on a molecular level, especially on the AR and MYC signaling pathways. 


\section{Results}

\subsection{MYC-Mediated Ribosomal Gene Expression Sensitizes Enzalutamide-resistant Prostate Cancer Cells to EP300/CREBBP Inhibitors}

Tobias Furlan, Alexander Kirchmair, Natalie Sampson, Martin Puhr, Martina Gruber, Zlatko Trajanoski, Frédéric R. Santer, Walther Parson, Florian Handle, Zoran Culig

Published in: American Journal of Pathology. March 08, 2021 DOI: 10.1016/j.ajpath.2021.02.017 (Online ahead of print)

Personal contribution: All experiments were planned, conducted, analyzed and visualized by the author. Exception were analyzation and visualization of patient data and screening for overrepresented Gene Ontology terms.

Summary: The second-generation anti-androgen Enzalutamide is frequently used for the treatment of advanced prostate cancer. However, development of resistance to enzalutamide occurs frequently. The AR co-activators P300 and CBP have been described to be involved in cellular processes of advanced prostate cancer. This study aimed to investigate the efficiency and role of P300 and CBP inhibitors in therapy-resistant prostate cancer. Indeed, enzalutamide-resistant cell lines were more sensitive to P300 and CBP inhibitors as compared to parental cells. This increased efficiency in resistant cells could not be explained solely by reduction of AR signaling. RNA-seq revealed that a subgroup of genes for ribosomal proteins as well as MYC signaling were reduced upon P300 and CBP inhibitors. Similarly, subgroups of genes for ribosomal proteins and MYC signaling were increased in enzalutamide resistant cells compared to parental cells. Regulation of genes for ribosomal proteins was confirmed for two candidates using western blot. The dependency of ribosomal protein genes on MYC signaling was confirmed by knockdown. Additionally, those 
results were validated in publicly available data of enzalutamide resistant cells, as well as patient data from the Stand Up To Cancer data set. To validate ribosomal proteins functionally, formation of ribosomes was targeted using CX5461, which prevents rRNA transcription. Enzalutamide-resistant cells were also more sensitive to CX5461 treatment. In summary, this study shows that P300 and CBP inhibitors affect expression of ribosomal proteins via MYC, which is a promising target in therapy resistant prostate cancer.

See Appendix for full-length article.

\section{2 p300 is upregulated by docetaxel and is a target in chemoresistant prostate cancer}

Martina Gruber, Lavinia Ferrone, Martin Puhr, Frédéric R Santer, Tobias Furlan, Iris E Eder, Natalie Sampson, Georg Schäfer, Florian Handle, Zoran Culig

Published in: Endocrine-related Cancer. 2020 March 27. DOI: 10.1530/ERC-19-0488

Personal contribution: Planning and data acquisition for viability analysis and IC50 calculation of CPI637 treated cells. Manuscript proofreading.

Abstract: Administration of the microtubule inhibitor docetaxel is a common treatment for metastatic castration-resistant prostate cancer (mCRPC) and results in prolonged patient overall survival. Usually, after a short period of time chemotherapy resistance emerges and there is urgent need to find new therapeutic targets to overcome therapy resistance. The lysine-acetyltransferase p300 has been correlated to prostate cancer (PCa) progression. Here, we aimed to clarify a possible function of p300 in chemotherapy resistance and verify 
p300 as a target in chemoresistant PCa. Immunohistochemistry staining of tissue samples revealed significantly higher p300 protein expression in patients who received docetaxel as a neoadjuvant therapy compared to control patients. Elevated p300 expression was confirmed by analysis of publicly available patient data, where significantly higher p300 mRNA expression was found in tissue of mCRPC tumors of docetaxel-treated patients. Consistently, docetaxel-resistant PCa cells showed increased p300 protein expression compared to docetaxel-sensitive counterparts. Docetaxel treatment of PCa cells for $72 \mathrm{~h}$ resulted in elevated p300 expression. shRNA-mediated p300 knockdown did not alter colony formation efficiency in docetaxel-sensitive cells, but significantly reduced clonogenic potential of docetaxel-resistant cells. Downregulation of p300 in docetaxel-resistant cells also impaired cell migration and invasion. Taken together, we showed that p300 is upregulated by docetaxel, and our findings suggest that p300 is a possible co-target in treatment of chemoresistant PCa.

See Appendix for full-length article. 


\section{3 shRNA Mediated stable Knockdown of P300}

Previous studies showed that P300 and CBP have some distinctive and some overlapping functions $(53,77)$. Other studies suggested that $P 300$ is more important in the pathogenesis of prostate cancer (66). One problem, which arises when studying P300 and CBP using inhibitors, is that it is impossible to discriminate between the two proteins as the inhibitors target both equally. Due to the high homology of bromo- and HAT domain, it is not possible to design specific inhibitors. An alternative to using inhibitors is knockdown, which specifically down-regulates the expression of only one protein. Knockdown therefore mimics the inhibition of only one of the two proteins. We wanted to explore if knockdown of P300 is sufficient to induce the same effects as inhibition of P300 and CBP via small molecular inhibitors. To examine the differences between P300 and CBP in the regulation of viability and AR signaling in our experimental background an inducible shRNA mediated knockdown of P300 was established. For this purpose the BLOCK-iT HiPerform Lentiviral PolII miR RNAi Expression System with emGFP from Invitrogen as described previously was used (78). For this purpose, LNCaP and DuCaP as well as respective resistant cells were first transfected with lentivirus packaged pHR-SFFV-rtTAM2-T2A-Puro plasmid and selected with puromycine. This plasmid expresses a doxycycline activator and repressor cassette. In the second step, those cells were transfected with a pLenti6.4 vector containing a shRNA for P300 or a scrambled control sequence and eGFP under control of a tetOn sequence and blasticidine resistance, which were also packaged by lentivirus previously. The doxycycline activator and repressor cassette and the respective inducible sequence were separated on two plasmids to reduce the chance of doxycycline-independent activation. Oligos coding for 
shRNA were purchased previously from Life Technologies (Hmi405238: shp300-1; Hmi405239: shp300-2). To verify successful transfection and doxycycline-dependent activation of the construct, cells were treated with different concentration of doxycycline and monitored for eGFP fluorescence (Figure 5.1), which was equal in all cell lines. To quantify efficacy of the P300 knockdown, cell lysates were blotted using antibodies specific for P300 (Figure 5.2). Both siRNA constructs were able to down-regulate P300 concentration-dependent in all cell lines. Knockdown of P300 was more pronounced in LNCaP EnzaR as compared to LNCaP cells. Effect on AR signaling was quantified by qRT-PCR of the AR targets FKBP5, TMPRSS2 and PSA (Figure 5.3). Interestingly, we could only observe in DuCaP EnzaR cells downregulation of multiple AR dependent genes upon shRNA mediated knockdown of P300. Lastly, also effects on viability were quantified using RealTime-Glo MT Cell viability assay (Promega) as described by the manufacturer (Figure 5.4). No significant changes in viability could be detected after shRNA mediated knockdown of P300. 

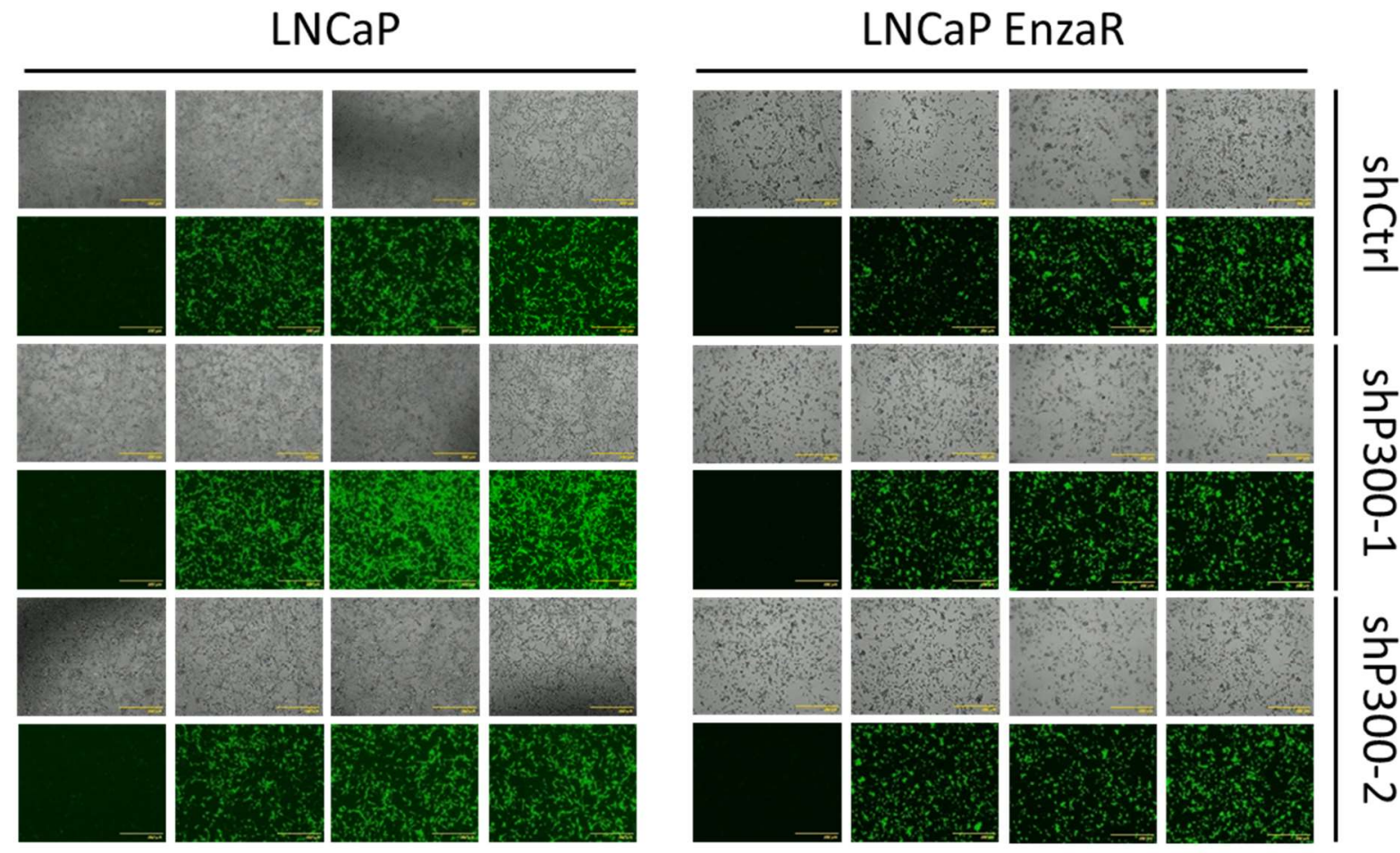

\section{DuCaP}

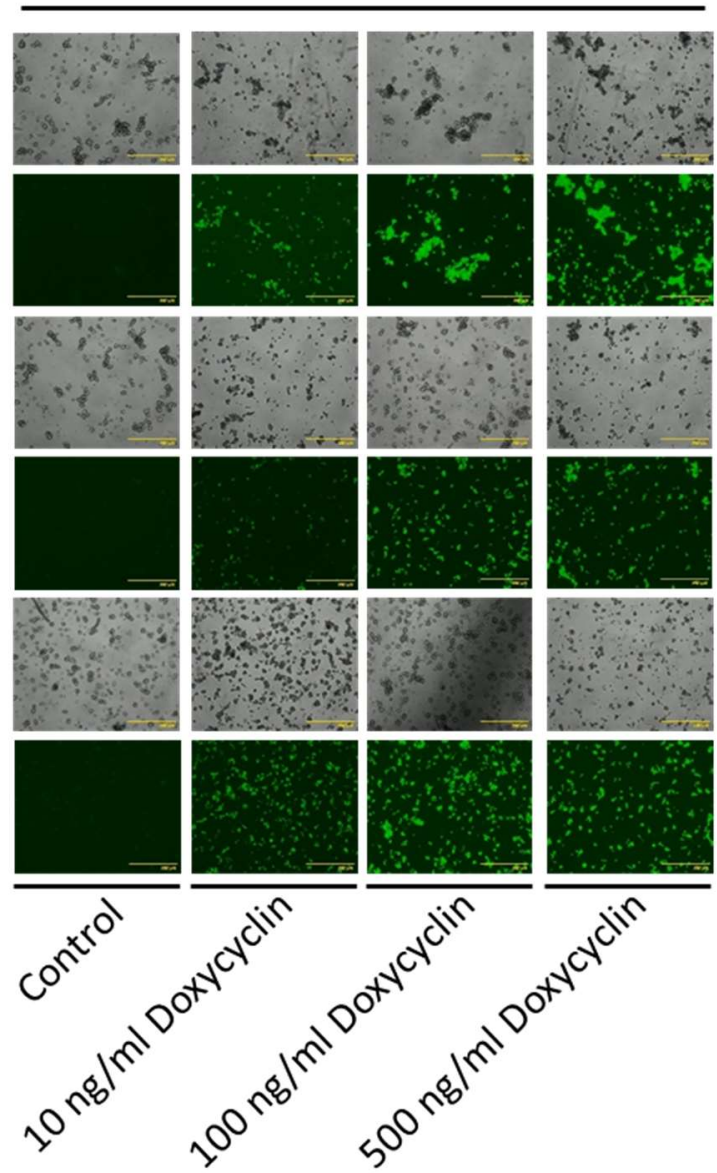

\section{DuCaP EnzaR}
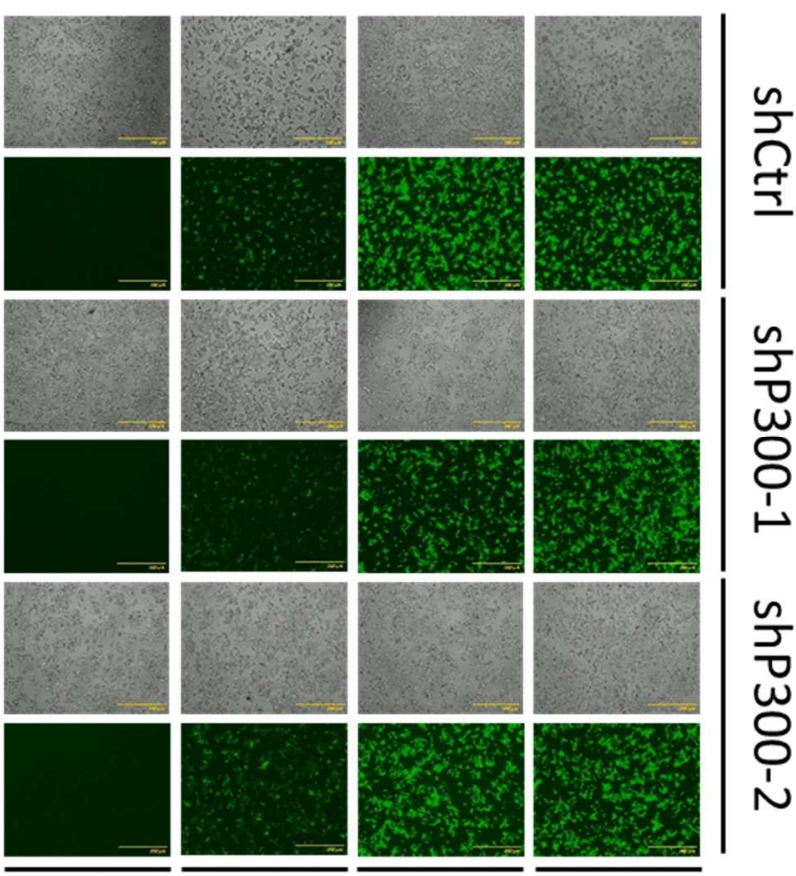

ก
0
$\omega$
0
0
1

$n$
0
0
0
0
1

Figure 5.1: Cells were treated with increasing amount of Doxycycline and imaged after three days, for bright-field or for eGFP fluorescence. 


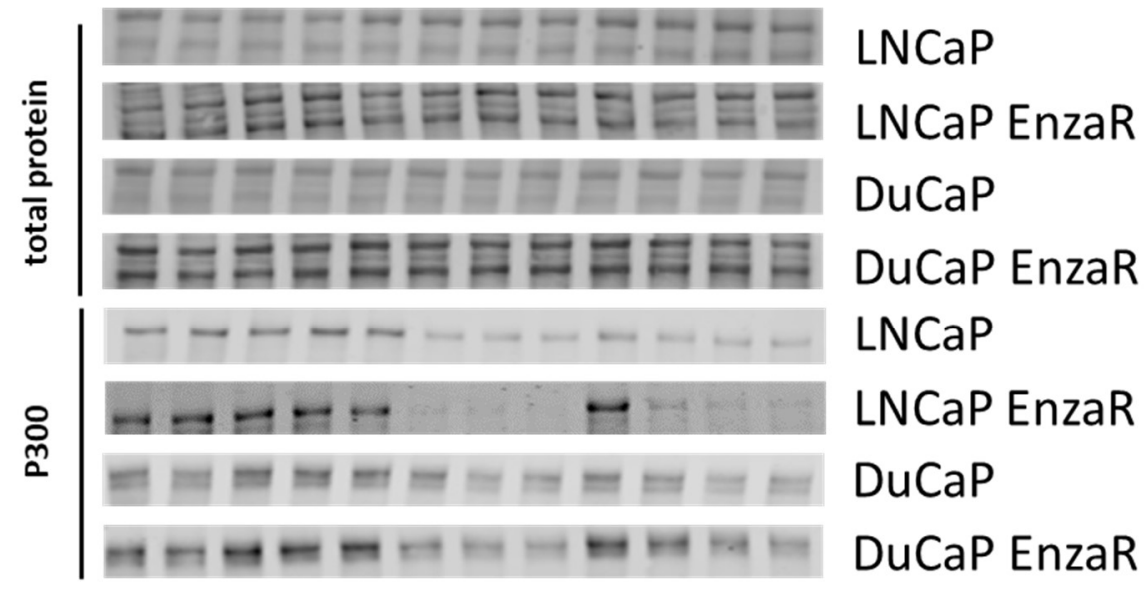

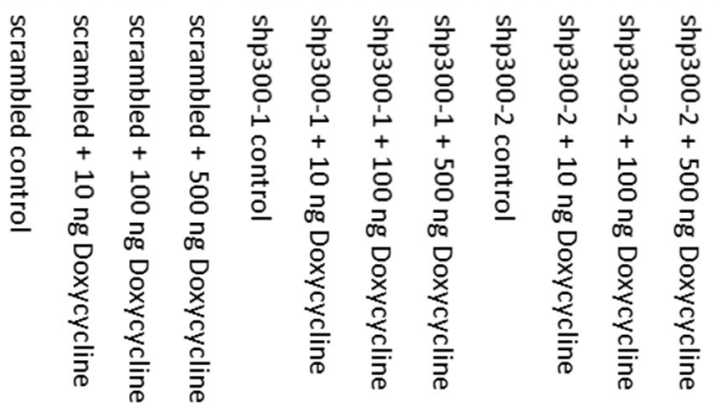

B

LNCaP
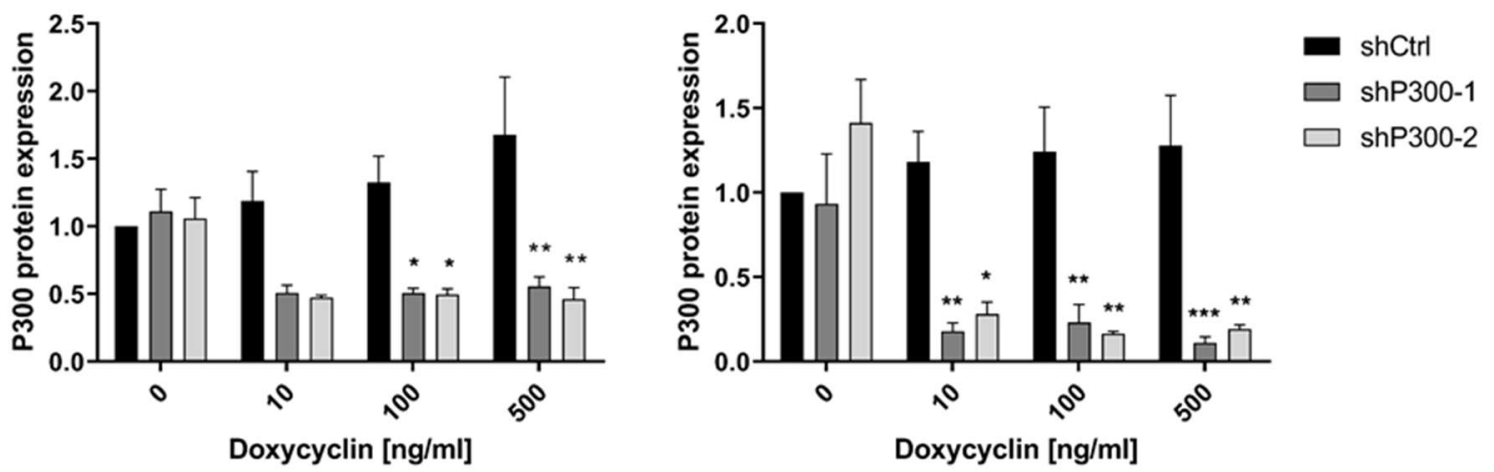

DuCaP

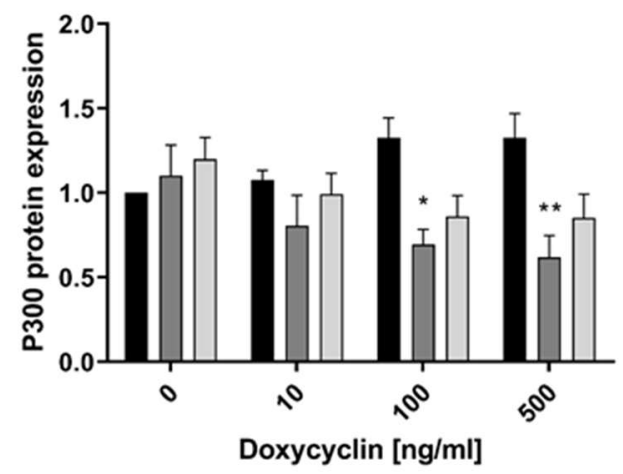

DuCaP EnzaR

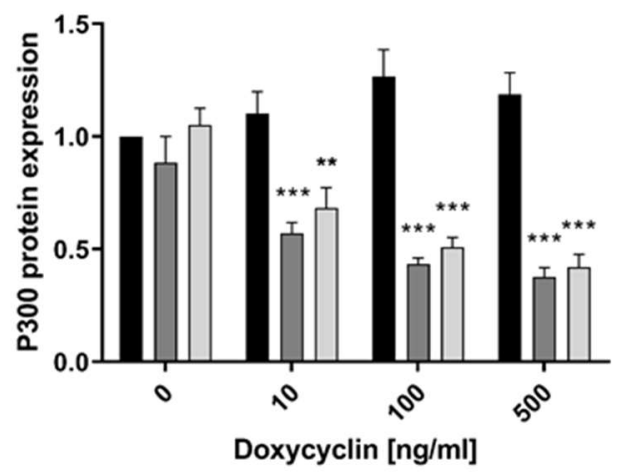

Figure 5.2: Before harvest, cells transfected with the different shRNA constructs were treated for $72 \mathrm{~h}$ with 10,100 or $500 \mathrm{ng} / \mathrm{ml}$ doxycycline or DMSO. (A) Representative western blots stained for 
total protein expression (top) or P300 expression. (B) Quantitative analysis of three independent experiments. Cells stably transfected with the P300 shRNA constructs were compared to cells stably transfected with a control construct. Values were normalized to respective DMSO treated controls. One-way-ANOVA with Bonferroni posttest was used. p-values of $<0.05,<0.01$, and $<0.001$ are denoted in figures by $* * *$, and $* * *$, respectively. 
A

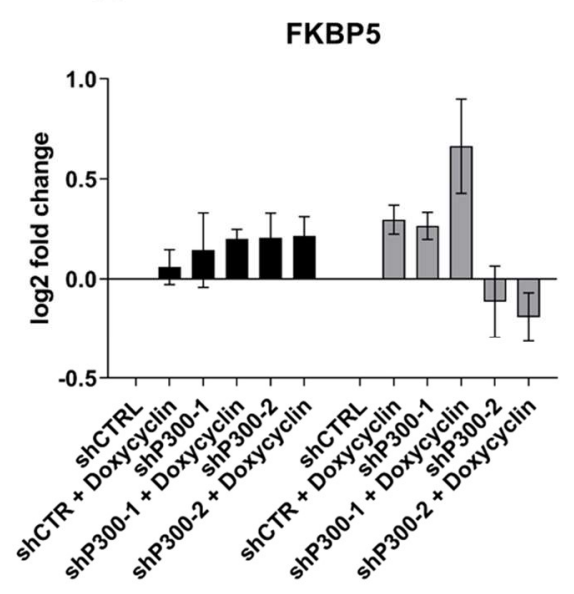

C

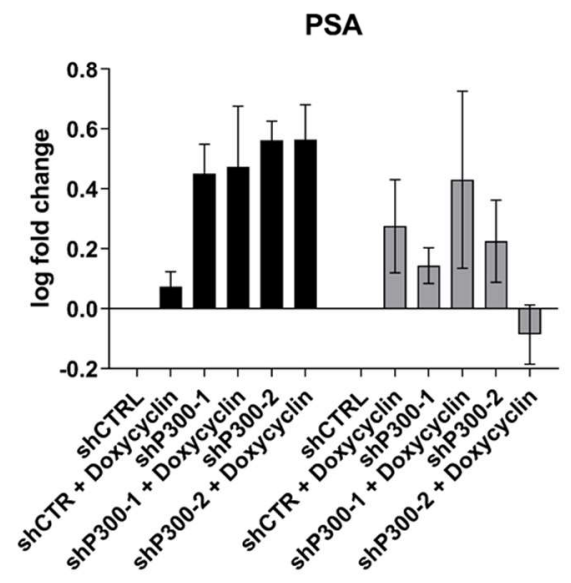

E
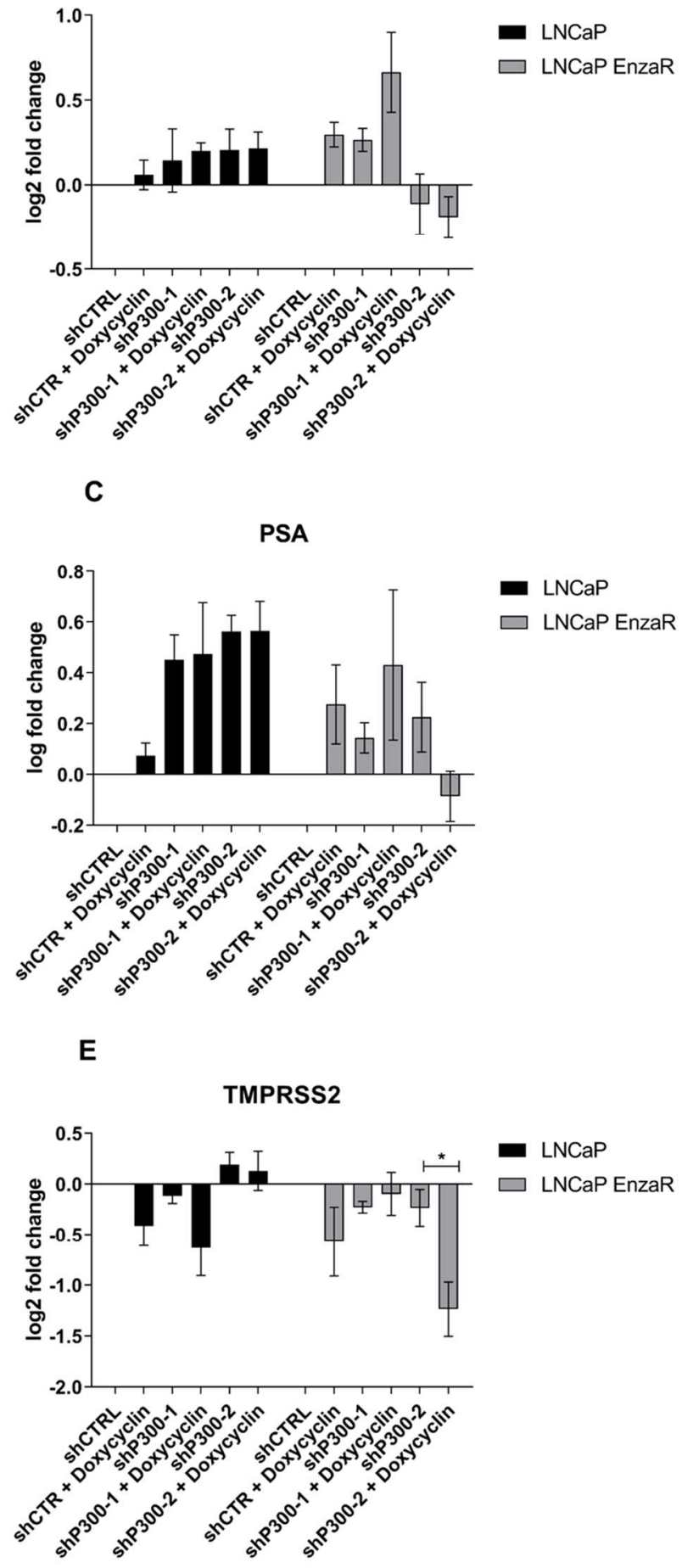

B

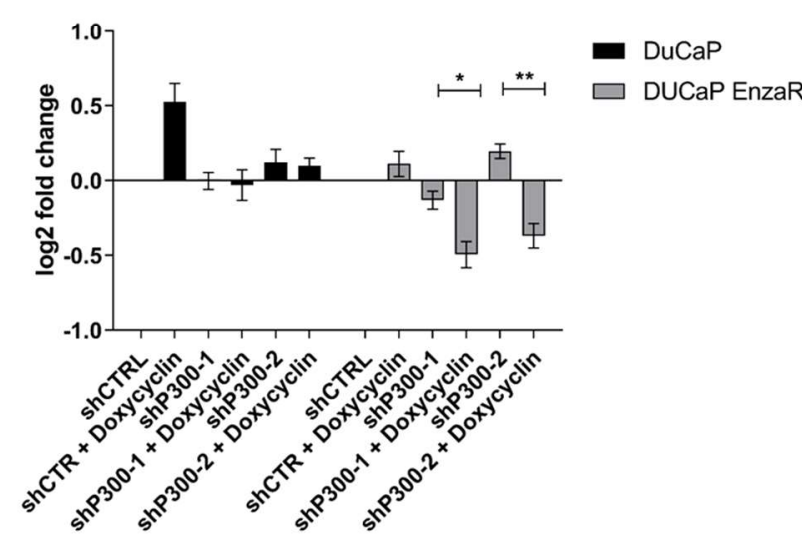

$\mathbf{F}$

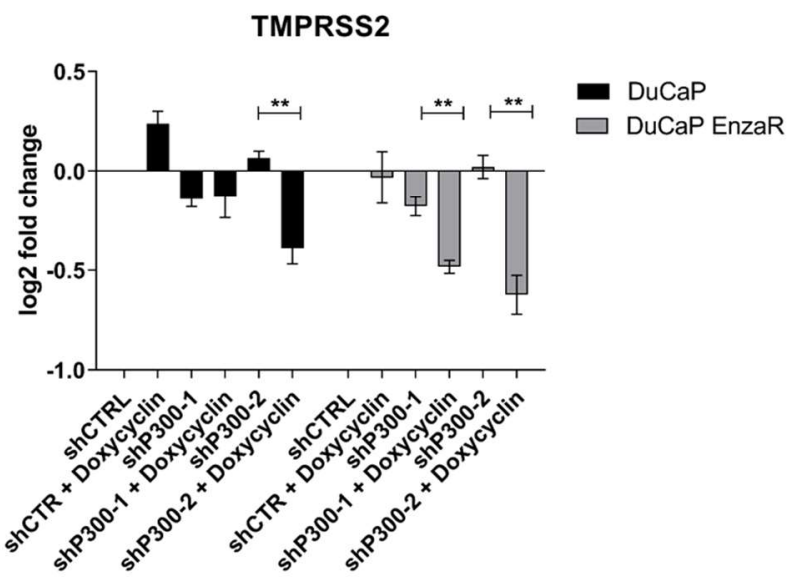

Figure 5.3: Before harvest, cells transfected with the different shRNA constructs were treated for $72 \mathrm{~h}$ with $100 \mathrm{ng} / \mathrm{ml}$ doxycycline or DMSO. qPCR results for parental and resistant DUCaP and LNCaP cell lines stably transfected with shP300 constructs or a control construct. Taqman probes specific for FKBP5 (A and B), PSA (C and D) and TMPRSS2 (E and F) were used. Values were normalized to respective DMSO treated 
control. Three independent experiments were analyzed statistically. One-way-ANOVA with Bonferroni posttest was used. p-values of $<0.05,<0.01$, and $<0.001$ are denoted in figures by $*, * *$, and $* * *$, respectively.

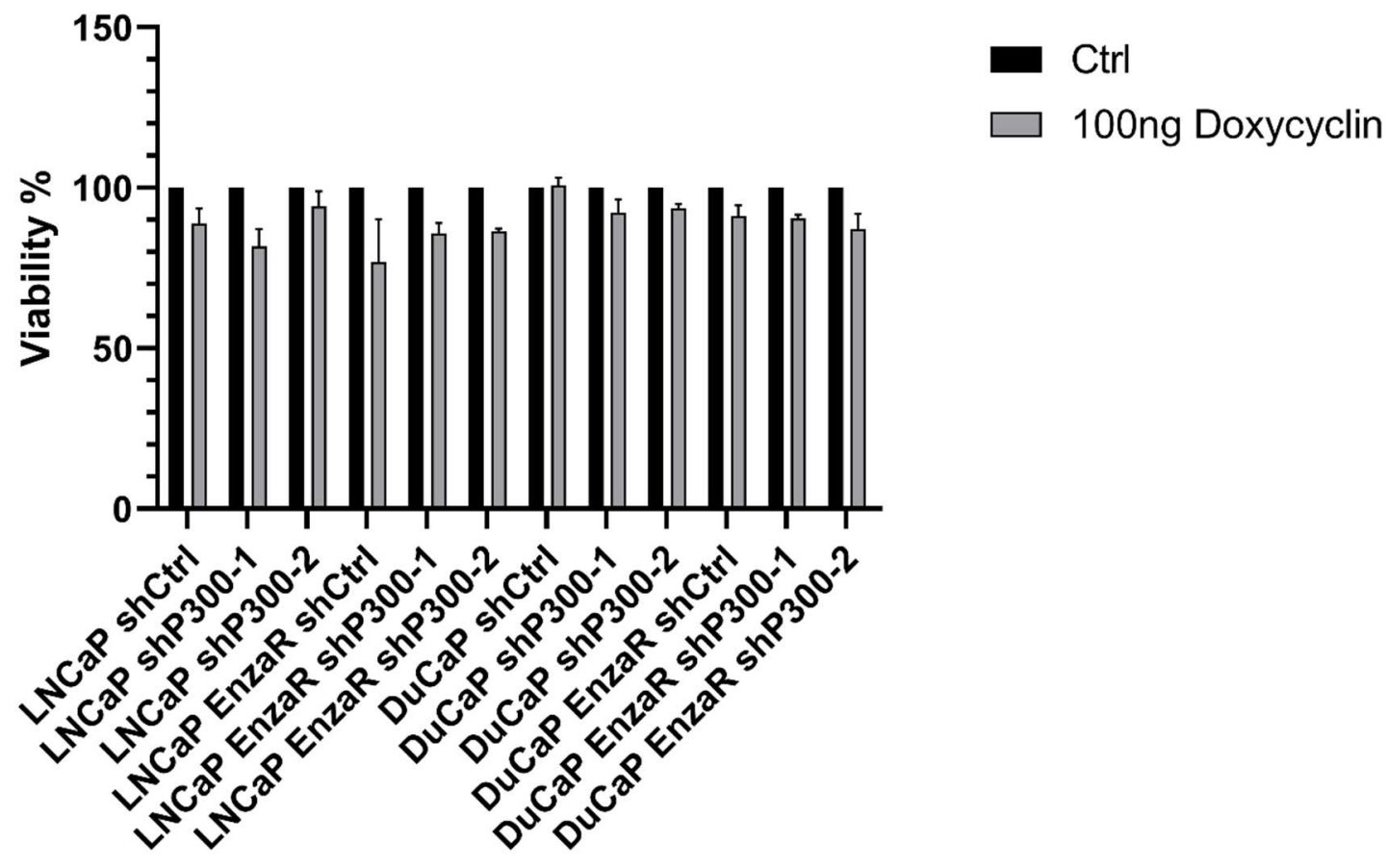

Figure 5.4: Parental and enzalutamide-resistant LNCaP and DUCaP cells stably transfected with a shP300 construct or a control construct were treated for $48 \mathrm{~h}$ with $100 \mathrm{ng} / \mathrm{ml}$ Doxycycline or DMSO. Viability was subsequently measured over $72 \mathrm{~h}$, doxycycline treatment was continued. Viability was normalized to DMSO treated control.

Contribution: Sandra Deichsler contributed work in data acquisition and maintenance of cell lines for the establishment of the conditional P300 knockdown. 


\subsection{WNT Activation in Enzalutamide-Resistant DuCaP Cells}

Epithelial to mesenchymal transition is a process that leads to increased invasive potential and therapy evasion of cancer cells. This process requires a high degree of cellular plasticity and is usually driven by WNT, TGF- $\beta$ and Notch signaling. Previous studies could show that epithelial to mesenchymal transition is also important for prostate cancer progression (79, 80). To determine if the enzalutamide-resistant cell lines also acquired a mesenchymal phenotype, cells were first cultured for 24 hours in growth medium or steroid free medium. Subsequently, cells were lysed, blotted and probed for N-cadherin, which is a mesenchymal marker as well as for loss of Vimentin, which is a marker for epithelial to mesenchymal transition (Figure 5.5 A). PC3 cells were used as a control. However, no gain of mesenchymal markers could be observed. Additionally, parental cells already lost expression of Vimentin. Nevertheless, GSEA showed that WNT signaling was significantly more active in enzalutamide-resistant DuCaP cells as compared to parental cells (Figure 5.5 B). This was verified for Cyclin D1, which is a target of canonical WNT signaling, using qPCR (Figure 5.5 C). $\beta$-catenin protein levels were also increased in the enzalutamide-resistant DuCaP cell line as shown by western blot (Figure $5.5 \mathrm{D}$ ). Those results correlate well as $\beta$-catenin enrichment is required for WNT signaling. Treatment with $10 \mu \mathrm{M}$ of either C646 or I-CBP112 for 24 hours could significantly reduce Cyclin D1 mRNA expression in the enzalutamide resistant cell line (Figure 5.5 E). However, only C646 has a significant impact on the canonical WNT activity, as determined by GSEA of enzalutamide-resistant DuCaP cells compared to parental cells (Figure 5.5 F). 
A

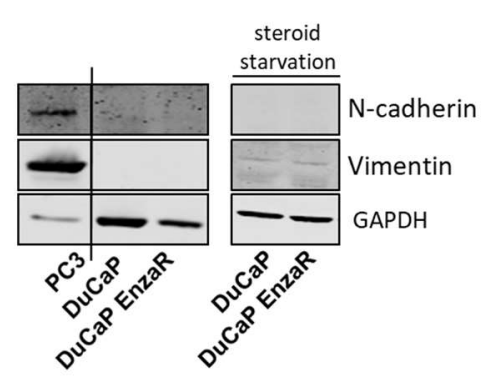

E

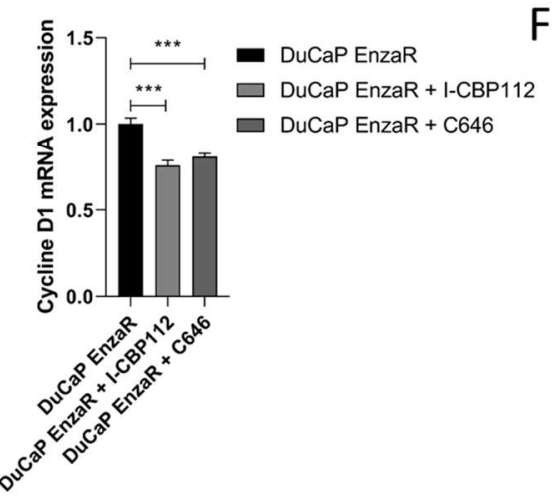

B

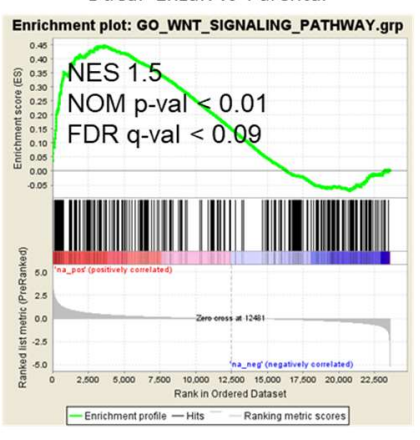

$\mathrm{F}$

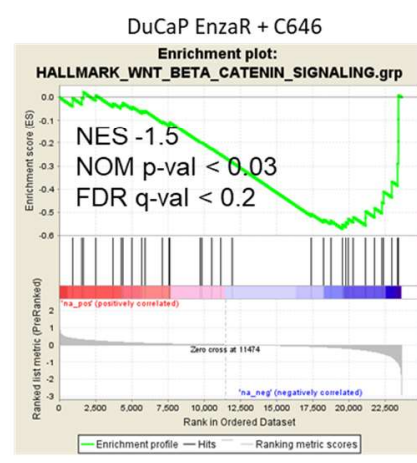

C
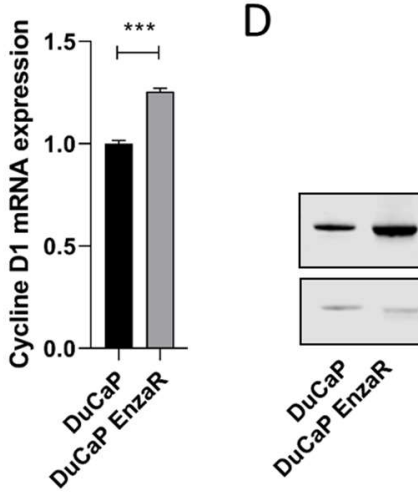

$\beta$-catenin $\alpha$-Tubulin

Figure 5.5: Representative western blot of parental and enzalutamide-resistant DuCaP cells grown for $\mathbf{2 4 ~} \mathrm{h}$ in either full growth medium or steroid free medium. PC3 cells are used as a positive control. Membranes were probed for N-cadherin, Vimentin and GAPDH as a loading control (A). GSEA of enzalutamide-resistant DuCaP cells compared to parental cells, using a WNT signaling signature (B). Cyclin D1 mRNA expression of parental and enzalutamide-resiant DuCaP cells, as determined by qRT-PCR. Statistics were calculated using t-test. $N=3$ (C). Representative western blot of parental and enzalutamide-resistant DuCaP cells probed for $\beta$-catenin and $\alpha$-Tubulin (D). Cyclin D1 mRNA expression, determined by qRT-PCR, of enzalutamide-resistant DuCaP cells upon treatment with $10 \mu \mathrm{M}$ of the respective inhibitor or DMSO equivalent for $\mathbf{2 4} \mathrm{h}$. Statistics were calculated using ANOVA with Bonferroni posttest. $N=3$ (E). GSEA of enzalutamide-resistant DuCaP cells treated with either $10 \mu \mathrm{M}$ of C646 or I-CBP112 for $24 \mathrm{~h}$, compared to untreated cells. A signature for $\beta$-catenin dependent WNT signaling was used (F). $p$-values of $<0.001$ is denoted in figures by $* * *$. 


\subsection{Protein Synthesis}

To investigate if $\mathrm{P} 300$ and CBP inhibitors reduce protein synthesis, protein neogenesis was quantified. This was done by incorporating a methionine analogue, which can be labeled by click-chemistry, into nascent proteins. Parental and enzalutamide-resistant DuCaP cells were pretreated with $10 \mu \mathrm{M}$ C646, I-CBP112 or equivalent volume of DMSO. Cells were then starved from methionine using methionine-free growth medium. The methionine analog azidohomoalanine was then added at a concentration of $25 \mathrm{nM}$ together with an inhibitor or DMSO treatment. Negative control cells were treated with $5 \mu \mathrm{g} / \mathrm{ml}$ cycloheximide to stop protein translation. After $24 \mathrm{~h}$ cells were fixated, permeabilized and a click-reaction with A488-labeled alkyne was performed. Additionally, cells were stained with propidium iodide to filter and exclude doublets in FACS analysis. A488 fluorescence was measured and blanked to samples treated without azidohomoalanine (Figure $5.6 \mathrm{~A}$ ). Interestingly, we found no differences between untreated and inhibitor-treated cells with the conditions used here. We observed a significant difference between parental and enzalutamideresistant cells, which correlates with different cell sizes (Figure 5.6 B and C). 
A

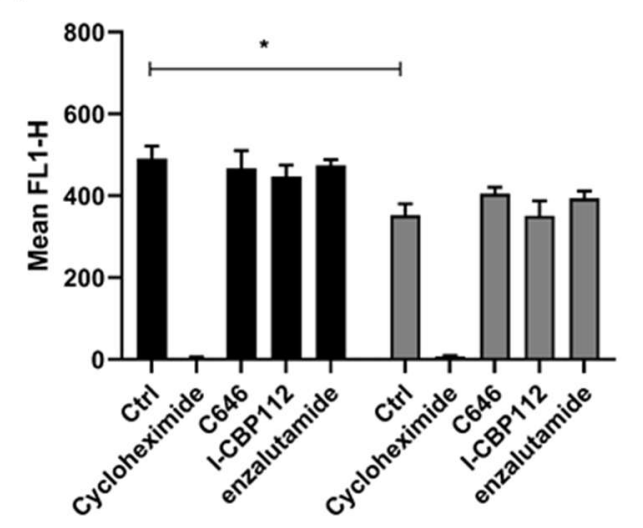

DuCaP

$\square$ DuCaP EnzaR

B

C

Cell Size

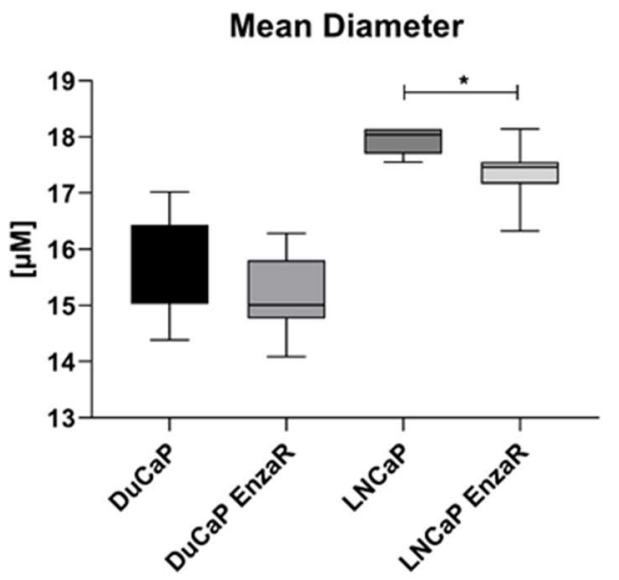

Figure 5.6: Incorporation of azidohomoalanine measured by mean fluorescence of click labeled Alexa488 in FACS analysis. Statistic was calculated using 2-way-ANOVA and Tukey's posttest. N=7 (A). Cell size measured by forward scattered light in FACS analysis. Statistics was calculated using t-test. $N=7$ (B). Diameter of cells measured using a CASY Counter. Statistics was calculated using t-test. $\mathbf{N}=7$ (C). p-values of $<0.05$ and $<0.01$ are denoted in figures by $*$ and $* *$ respectively. 


\section{Discussion}

Development of therapy resistance occurs after enzalutamide treatment, ADT and chemotherapy. Resistance to ADT and Anti-androgen treatment is most commonly characterized by reactivation of AR signaling. Several ways how P300 and CBP contribute to AR signaling and resistance to ADT and AR inhibition are known.

P300 and CBP are not only needed as structural components of the AR complex but their function as HAT is detrimental to AR signaling (81). It was shown that P300 expression correlates with growth of prostate cancer in cell line models as well as with tumor volume in patient samples (82). While P300 expression is negatively regulated by AR signaling an increased expression was observed in cell exposed to low androgen levels (66). Furthermore, P300 is needed for IL6-mediated androgen-independent activation of the AR (67). Overexpression of other nuclear receptors like the glucocorticoid receptor in response to anti-androgen treatment has also been demonstrated (83). Like with other nuclear receptors, activity of the glucocorticoid receptor is positively regulated by P300 (84). Involvement in androgen-independent activation of the AR together with higher expression under ADT conditions make P300 a key element in ADT resistance. A role in therapy resistance was also attributed to the $\mathrm{P} 300$ homologue CBP. CBP is, similar as P300, upregulated upon androgen withdrawal (85). However, $C B P$ is, in contrast to P300, not involved in IL6-mediated activation of the AR. Instead, CBP is involved in reactivation of AR signaling after treatment with the anti-androgen hydroxyflutamide (86).

In recent work, our group could show that P300 is a target involved in chemotherapy resistance (78). P300 is increased in docetaxel-resistant and -sensitive cell line models as 
well as in patient tissue upon docetaxel treatment. This increase of P300 protein is mediated via enhanced stability, because there are no changes on mRNA level. Down-regulation of P300 leads to reduced colony formation in docetaxel-resistant cells but not in docetaxelsensitive cells. When monitoring proliferation capability, however, no differences could be observed between docetaxel-resistant and -sensitive cells. Interestingly, P300 but not CBP seems to be involved in docetaxel resistance. This is in contrast to what was observed here in enzalutamide-resistant cells upon inhibitor treatment. Enzalutamide-resistant cells showed a significantly higher sensitivity towards inhibition of P300 and CBP than parental cells. Down-regulation of only P300 in enzalutamide-resistant cells using siRNA was not sufficient to reduce viability, suggesting that CBP is involved. Due to the high homology between P300 and CBP most of their functions are overlapping. Both P300 and CBP are upregulated upon steroid depletion and involved in MYC signaling $(66,85)$. Nevertheless, also not overlapping functions were identified, like different affinities for binding to transcription factor binding sites related to AP-1, AP-2, SP1, E2F and SRF (87). Differences between P300 and CBP are especially evident for AR mediated signaling. It was shown that knockdown of P300 results in down-regulation of many AR signaling dependent genes, which could not be compensated by CBP. Knockdown of CBP on the other hand has little effect on the expression of AR signaling dependent genes (88). Considering this, it is conceivable that P300 and CBP carry unique functions in therapy resistance, dependent on the signaling pathways involved.

Although interesting, it is currently not feasible to address different functions of P300 and CBP solely using inhibitors. Due to the high homology of the HAT- and bromo-domain it is currently impossible to develop small molecular inhibitors specific for either P300 or CBP. 
Furthermore, it has been shown that inhibition of P300 and CBP by small molecular inhibitors and knockdown does not necessarily impact the same pathways (89). Nevertheless, major efforts to develop inhibitors for P300 and CBP were made in the last 10 years. Starting with naturally occurring compounds like garcinol and curcumin, which showed inhibitory potential for P300 and CBP, several small molecular compounds were developed and optimized over the years. Bottlenecks for the development of small molecular inhibitors are not only specificity and potency but also appropriate effectiveness in vivo. Targeting the HAT domain and the bromo domain is considered of equal importance, but requires different chemical platforms. Inhibitors targeting the bromodomain of P300 and CBP are mostly derivates of benzimidazole, piperidine, benzodiazepinone and indoles (90). Benzimidazole is a ring-like compound consisting of a benzene and an imidazole. Benzimidazols are the basis of different biological active compounds. Initial efforts led to development of compounds targeting the bromodomain of CBP 22-fold over other BRD proteins. This compound could be improved further resulting in the CBP30 compound, which displays 40-fold selectivity for CBP over other BRD proteins (91). Here we used ICBP112 which was has a related benzo-oxazepine basis and was discovered upon screening of a compound library (89). More recently P300 and CBP inhibitors deriving from benzooxazepin have been discovered and improved further, but they are not commercially available (92). In 2017, a P300 and CBP inhibitor with a benzodiazepinone base was discovered through screening of a fragment collection using a thermal shift assay. Further development resulted in the CPI637 inhibitor. This inhibitor shows high potency and a specificity of $>700$-fold over other BRD proteins, which was the highest at this time point (93). Therefore, CPI637 was also included in this work. Finally, a recently reported inhibitor, CCS1477, has all properties required for use as drug. This compound is a derivative of 
benzimidazole and can be administered orally. CCS1477 is highly potent while still being specific for P300 and CBP and also passed safety screen of various receptors, enzymes and ion channels (65). For HAT domain specific inhibitors, different molecular bases, including derivatives of pyrazolone, oxazolidinedione, barbituric acid, indoles and alkaloids exists. Here we used C646, which is a pyrazolone derivative (94). This compound was discovered through a virtual ligand screen, which was made possible by the high resolution $\mathrm{x}$-ray structure data of P300/CBP in complex with a substrate analogue (95). Another HAT domain specific inhibitor derivative of oxazolidinedione with higher potency and specificity was reported later in 2017. This inhibitor was also discovered through a virtual ligand screen and was subsequently optimized substantially, resulting in a 1000-fold potency as compared to C646 (96). However, no HAT domain specific inhibitors with drug like properties have been reported yet.

Many previous studies could show that P300 and CBP are important for prostate cancer due to their co-activation of the AR $(82,97)$. Additionally, P300 and CBP are up-regulated upon anti-androgen treatment and androgen withdrawal thereby sensitizing cells to lower levels of androgen or to ligand-independent activation of the $\operatorname{AR}(66,67,98)$. Here we are interested in resistance after enzalutamide treatment. In clinics, enzalutamide is mainly used for castration-resistant metastatic prostate cancer. Recently it became evident that enzalutamide is also effective in hormone-sensitive prostate cancer (99). As with other therapies, a resistance to enzalutamide treatment develops frequently. We therefore initially hypothesized that P300 and CBP are valid targets in enzalutamide-resistant prostate cancer due to the interactions with the AR. The particular cell lines used here were generated previously by chronic treatment with increasing concentrations of enzalutamide 
$(83,100)$. The cell lines also display a reactivation of AR signaling. The DuCaP cells have an amplification of the AR and express it at high levels. The DuCaP cell line also expresses the AR variant 7, which is associated with therapy resistance (101). The LNCaP cell line expresses a mutant AR (T877A). Our results show a strong increase of AR activity upon long-term enzalutamide treatment.

Despite development of resistance, we do not observe an increase in P300 or CBP protein expression, which is in line with previous results (65). Nevertheless, we show here in a viability assay that enzalutamide-resistant cells are more sensitive towards P300 and CBP inhibitors targeting either the bromo-domain or the HAT-domain. Conversely, downregulation of AR activity may not be the prime reason. While the P300 and CBP inhibitors decrease AR activity, the inhibition of the AR activity is comparable to that by enzalutamide, which became apparent when analyzing FKBP5 protein expression upon inhibitor or enzalutamide treatment. Considering those cell lines are resistant to enzalutamide this might not affect proliferation. Especially in the LNCaP cell line it is clear that the increase of AR activity gained with resistance cannot be reduced via P300 and CBP inhibition like in parental cells. Adaptions to AR signaling in response to ADT and enzalutamide treatment might therefore prevent effective inhibition of the AR signaling by P300 and CBP inhibitors. An explorative RNA-seq revealed that MYC activity was reduced upon treatment with the bromo- or HAT- domain inhibitor in the enzalutamide-resistant DuCaP and LNCaP cell lines. MYC activity was up-regulated in the LNCaP EnzaR cells compared to parental cells and in DuCap cells when compared to enzalutamide-treated parental cells. MYC is one of the most common oncogenes that regulates approximately $15 \%$ of all genes (102). MYC is also commonly overexpressed in prostate cancer (103). The exact way by which MYC impacts 
prostate cancer growth is less clear. Previous work showed that MYC has the potential to initiate malignancies, as MYC overexpression is sufficient to immortalize prostate cells in vitro (104). Furthermore, MYC activity correlates with AR full length and variant expression and is thus involved in late stage and therapy-resistant prostate cancer $(105,106)$. MYC amplification is commonly observed after androgen deprivation therapy and may induce AR- independent resistance to therapy $(107,108)$. Together with our results, we consider MYC signaling a crucial part of enzalutamide resistance. Those results correlate with findings in other anti-androgen resistant cell lines generated by another group (109).

Importantly, it has been shown that P300 and CBP directly interact with and acetylate MYC (110). Furthermore, P300 and CBP also acetylate chromatin at the location of MYC target genes (111). In our experimental background, we can show that both the HAT domain inhibitor C646 as well as the bromodomain inhibitor I-CBP112 inhibit MYC activity but not MYC protein or mRNA expression. Counterintuitively, MYC mRNA and protein were increased by C646 treatment. On the other hand, MYC protein was decreased upon treatment with the newer generation bromodomain inhibitor CPI637. The relations between P300/CBP and MYC are complex. Previous studies attributed binding of P300 and CBP to MYC with increased activity and increased stability. Acetylation of MYC, on the other hand, was correlated to decreased stability (111). P300 and CBP inhibitors generally suppress MYC activity (65). However, total MYC protein is not the limiting factor for inhibition of MYC activity by P300/CBP inhibitors. A previous study showed that an inhibitor targeting the HAT domain could not decrease MYC levels while an inhibitor of the bromo domain decreased MYC at mRNA levels, despite the fact that both equally suppressed MYC activity (112). 
RNA-seq revealed also that expression of ribosomal proteins, which are downstream of MYC signaling, are significantly regulated in our experimental background. We can show that approximately $60 \%$ of ribosomal proteins are up-regulated in enzalutamide-resistant DuCaP and LNCaP cells as compared to parental cells. Overexpression of ribosomal proteins and activation of MYC signaling were observed in enzalutamide-resistant DuCaP and LNCaP cells despite differences in regulation of AR activity. siRNA-mediated knockdown of MYC in enzalutamide-resistant cells proved to be sufficient to reverse ribosomal protein expression. Additionally, we could show that the regulation of ribosomal proteins by MYC is independent of AR signaling as knockdown of MYC reduced ribosomal protein expression also in the AR-negative cell line PC3. The subset of up-regulated ribosomal proteins differed between DuCaP EnzaR and LNCaP EnzaR. DuCap EnzaR and LNCaP EnzaR shared 13 of the up-regulated ribosomal proteins. Overexpression of ribosomal proteins in anti-androgenresistant cells was confirmed in a publicly available RNA-seq data of two AR independent, anti-androgen-resistant cell lines generated by another group. Both of those cell lines are derived from LNCaP, one chronically treated with enzalutamide and the other with RD-162, which is an anti-androgen closely related to enzalutamide (109). Treatment with bromodomain or HAT inhibitors of P300 and CBP reverses the changes imposed by chronic enzalutamide treatment and down-regulates some ribosomal proteins. Up-regulation of ribosomal proteins in enzalutamide-resistance and down-regulation by P300 and CBP inhibitors was confirmed for two ribosomal proteins on western blot. Ribosomal biogenesis, a target of oncogenic MYC, includes ribosomal proteins and ribosomal RNA (113). Ribosomal biogenesis is tightly related to cell proliferation. Increased ribosomal biogenesis by MYC is associated with cancer. Unchecked synthesis of proteins to sustain uncontrolled cancer growth is considered a hallmark of cancer (114). Increased number of ribosomes are 
frequently observed in the form of hypertrophied nucleoli, as ribosomal subunits are assembled there. A previous study showed that MYC promotes cancer initiation by increased protein synthesis through an increased number of ribosomes. This malignant phenotype could be reversed by limiting expression of single ribosomal proteins (115). Ribosomal action is tightly regulated in normal cells by impaired ribosome biogenesis checkpoint (IRBC). The IRBC triggers cell death upon ribosomal stress, which could be induced by aberrant ribosomal biogenesis. Since it was shown that IRBC is mediated by the tumor suppressor p53 (116), it is important to note that both cell lines used here contain some sort of p53 impairment. The LNCaP cell line expresses p53 at low levels and the DuCaP cell line carries a mutation in the TP53 gene (117).

Our bioinformatics results showed that many ribosomal proteins are overexpressed in the enzalutamide-resistant cell lines. However, for approximately one third of ribosomal proteins we could not observe an increased expression. Therefore, those changes might not necessarily lead to the formation of additional ribosomes with normal distribution of ribosomal proteins. In contrast to increased number of ribosomes, recent studies could also show that overexpression of single ribosomal proteins is sufficient to affect proliferation, invasion and metastatic potential of cancer. It has been shown that RPL19 is frequently upregulated in prostate cancer (118). Knockdown of ribosomal protein L19 (RPL19) is sufficient to abrogate the aggressive phenotype of PC-3 cells in vivo (119). Interestingly, that work suggests that down-regulation of RPL19 does not abrogate ribosomal function. Instead, RPL19 may have extra ribosomal function. RPL15 is similarly related to metastatic potential and worse patient outcome in breast cancer (120). Another way how changes in expression of single ribosomal proteins can impact gene expression is via the formation of 
heterogeneous ribosomes (121). Transcription of specific sub-pools of genes by heterogeneous ribosomes has been shown for RPL38, RPL21, RPL5 and RPL10A (122-125). Together these results suggests that ribosomal proteins play a crucial role in MYC-driven cancers, although they are not always part of ribosomes. Whether subgroups of ribosomal proteins have redundant functions in cancer or therapy resistance remains to be investigated.

Importantly, we show that it is possible to target ribosomal proteins in the enzalutamideresistant cell lines by targeting MYC using P300 and CBP inhibition. Targeting ribosomal proteins directly is promising in MYC-driven cancers, as shown previously (126-128). This might also be true for prostate cancer and specifically enzalutamide-resistant tumors, which show an up-regulation of ribosomal proteins. Here we used CX-5461 to target ribosomes in enzalutamide-resistant prostate cancer directly. CX-5461 binds to G-quadruplex DNA, which is a secondary structure consisting of several layers of tetrads formed by four sections of guanine rich DNA and stabilized by guanine hydrogen bonds (129). G-quadruplex DNA is formed at the GC rich genomic loci of rRNA and is required for efficient transcription by polymerase I. Binding of CX-5461 to G-quadruplex DNA inhibits polymerase I function, abrogates rRNA transcription and thus stops ribosomal biogenesis $(130,131)$. Similar as with P300 and CBP inhibitors we can observe a significantly lower IC50 for CX-5461 in enzalutamide-resistant cells as compared to parental cells. Additionally, we show that treatment with CX-5461 significantly reduces migration of enzalutamide-resistant LNCaP cells.

Regulation of ribosomal proteins by MYC was also confirmed in patient samples. Publicly available RNA-seq and genomic data from metastatic CRPC of the SU2C dataset revealed 
aberrations in mRNA expression and copy number. We can show that in patients mRNA expression of ribosomal proteins is significantly positively correlated with MYC activity. Interestingly, DNA data of patients reveal an additional regulation of MYC and ribosomal proteins on the genomic level. Many ribosomal proteins and MYC are commonly amplified. Especially MYC, RPL7, RPL30, RPL8, and RPS20 are often found to be highly amplified together. This is not surprising since all of those genes are located in proximity on chromosome $8 q$. Approximately $27 \%$ of patients carry those amplifications, which suggests an important function. Previous studies showed that the amplification of chromosome $8 \mathrm{q}$ are common in advanced prostate tumors but not in primary prostate tumors. Amplifications of chromosomes $8 \mathrm{q}$ were also associated with worse outcome (132). Chromosome $8 \mathrm{q}$ gains are not limited to prostate cancer but also frequently observed in other malignancies like renal cell carcinoma, pleuropulmonary blastoma and gastric carcinoma (133-135). Other genes for ribosomal proteins, which are not located on chromosome 8 , were also highly amplified in up to $10 \%$ of patients. Therefore, data from patient samples suggests that ribosomal proteins are up-regulated by MYC activity and by copy number aberrations (CNA) of the genes.

It should be mentioned that changes observed in resistant cells show different ways how cells can adapt to treatment. Other cell lines generated in our lab, which are resistant to docetaxel treatment, show gain of stem cell-like phenotype as well as epithelial to mesenchymal transition. Epithelial to mesenchymal transition is generally associated with worse outcome and related to therapy resistance $(136,137)$. In the docetaxel-resistant cell lines those changes were induced by the downregulation of miR-200 and miR-205 expression, which are repressors of ZEB1 and ZEB2 (138). ZEB1 and ZEB2 are negative 
regulators of E-cadherin, which in turn suppresses WNT signaling and promotes cell-cell contacts. We cannot observe expression of $\mathrm{N}$-cadherin, which is a typical marker for epithelial to mesenchymal transition or vimentin expression in our enzalutamide-resistant cell line models. However, there is an increased WNT activity in enzalutamide-resistant DuCaP cells as compared to parental cells. This correlates with increased $\beta$-catenin protein levels, which is limiting for WNT signaling and expression of the WNT target cyclin D1. Cyclin D1 is an important regulator of the transition from the G1-phase to S-phase and is therefore not only often upregulated in cancer but also associated with worse outcome in patients $(139,140)$. Previous studies showed that WNT signaling is not only involved in the formation of cancer stem cells (141), but also in therapy resistance of prostate cancer $(142,143)$. Important to note here is that canonical WNT signaling is mediated via transcription factors TCF/LEF family, which require P300 and CBP as co-activators. We can show that inhibition of P300 and CBP via C646 and I-CBP112 significantly reduces cyclin D1 expression. However, RNA-seq results suggest that only C646 has substantial effect on canonical WNT activity. More research is required to determine if P300 and CBP inhibitors do have a meaningful impact on WNT signaling in this context, especially considering that non-canonical WNT signaling might not be affected. A recent study could show that abiraterone acetateresistant cell models are also more sensitive to P300 and CBP inhibitors. Abiraterone acetate is, similar as enzalutamide, used to treat metastatic ADT resistant prostate cancer. In contrast to enzalutamide, abiraterone acetate does not target the AR directly instead, it inhibits CYP17A1, which is an enzyme required for the synthesis of DHT. Several prostate cancer cell line showed increased expression of the transcription factor CREB1 upon chronic abiraterone acetate treatment. Additionally, expression of CREB1 was sufficient to induce abiraterone acetate resistance (144). CREB1 exerts its transcriptional function through P300 
and CBP, which sensitizes CREB1-dependent cells to P300 and CBP inhibitors (145). Finally, a recent study showed that P300 and CBP protein overexpression does not necessarily correlate with castration resistance. However, high protein expression of P300 was predictive for worse overall survival and time to therapy resistance. They could also show that bromodomain inhibition of P300 and CBP reduces not only AR but also MYC signaling in mouse models and patient derived xenografts, which is in line with our results. Interestingly, also AR-V7 signature, which was related to therapy resistance before, was significantly reduced upon inhibitor treatment (65). A recent study where other enzalutamide-resistant cell models were used could show that non-canonical AR targets are expressed in resistant cell lines. In those enzalutamide-resistant models, expression of several canonical AR targets was inhibited, while depletion of AR was still effective. Several genes including ID1, PFN2, and ID3, which are ARE independent, non-canonical AR targets where overexpressed. Expression of those genes is necessary for enzalutamide-resistance in those cell lines and is associated with worse outcome in patients, which underwent enzalutamide therapy. The protein CXXC5 directs AR to promotors of the non-canonical targets, which are rich in cytidine-phosphate-guanosine dinucleotides but do not contain ARE. Binding of CXXC5 is also positively correlated to acetylated H3K27. Therefore, those cell lines were also significantly more susceptible to inhibition to a bromodomain inhibitor of P300/CBP and BRD proteins (146).

Taken together, our results show that P300 and CBP are reliable targets for enzalutamideresistant prostate cancer. Interestingly, reduction in AR activity might not be the limiting factor in enzalutamide-resistant cells under p300/CBP inhibitor treatment, as was noted by previous studies. Suppression of the MYC-mediated expression of ribosomal proteins 
instead likely leads to the increased efficiency of P300 and CBP inhibitors observed in enzalutamide-resistant prostate cancer. This is in line with previous studies from our lab, which concluded that PC3 cells, which do not express AR, are also affected by the P300/CBP HAT inhibitor C646 (73). Those findings attribute an important role to subgroups of ribosomal proteins in therapy-resistant prostate cancer and open the possibility to investigate the exact mechanism in future studies. Additionally, ongoing clinical trials with bromodomain inhibitors in prostate cancer also show promising results and demonstrate low side- and cytotoxic effects (65). Combined with continuing efforts to improve specificity and potency of P300 and CBP inhibitors, powerful new therapeutics for previously untreatable therapy resistant prostate cancer will likely be established in the future. 


\section{Outlook}

P300 and CBP are involved in a multitude of signal pathways. Our results as well as previous studies could show that targeting P300 and CBP leads to inhibition of several signaling pathways, which are involved in the development of resistance. Initial studies emphasized the suppression of AR signaling of P300 and CBP inhibitors $(61,147)$. In our cellular models both AR signaling and the MYC/ribosomal protein axis reduced upon inhibitor treatment. Additionally, we can show that P300 and CBP inhibitors have the potential to reduce WNT signaling. Future studies with appropriate models for WNT are required to validate those results. In a recent study, P300 and CBP inhibition proved also more efficient in enzalutamide-resistant C4-2 cells as compared to parental cells. However, they attributed this effect to destabilization of the histone demethylase JMJD1A, which induces tumor growth via AR and MYC signaling (148). Taken together, different oncogenic signaling pathways as well as therapy resistance mechanisms converge in P300 and CBP. P300 and CBP inhibitors therefore carry a high potential as therapeutic option in prostate cancer and specifically enzalutamide-resistant prostate cancer. Since most evidence comes from cell line experiments future research in animal models and with patient samples is required to determine the efficiency of targeting the before mentioned signaling pathways by P300 and CBP inhibitors also in vivo.

Since the start of this project, novel P300 and CBP inhibitors have been discovered and newer generation of inhibitors became available (149-151). Newer generation P300 and CBP inhibitors generally exhibit an improved specificity and potency, which is important for clinical applications. Not all inhibitors are specific for P300 and CBP. Some inhibitors which target the bromodomain are also active against other bromodomain containing proteins of 
the BRD family, which also promote cancer growth (152). However, for prostate cancer there is no data available comparing P300 and CBP-specific inhibitors and inhibitors, which also target proteins of the BRD family. Targeting a broader group of proteins might also induce more unwanted effects.

Pharmaceutical companies have also recognized the high potential of P300 and CBP inhibitors for prostate cancer. Two clinical studies with different P300 and CBP inhibitors have been started. For one clinical trial CCS1477, a P300 and CBP bromodomain inhibitor, is used and for the other clinical trial ZEN-3694, a pan bromodomain inhibitor is used. Preliminary data available from the CCS1477 study show a reduction or stabilization of blood PSA concentration as well as reduced amount of nuclear MYC upon inhibitor treatment. Complete data will be available later this year (65). This clinical trial is not only a dose escalation study but also contains two arms with co-treatments with either enzalutamide or abiraterone acetate. Currently, little data for P300 and CBP inhibitors in combination with other treatments is available. However, non-significant synergistic effects by enzalutamide and CCS1477 in a therapy-resistant cell line have been reported (65). Therefore, it is conceivable that combination therapy of P300 and CBP inhibitors and enzalutamide improves outcome. Similarly, a synergy was observed in LNCaP cells for P300 and CBP inhibitor treatment combined with abiraterone acetate treatment (144). Another study could show that treatment with the P300 and CBP HAT inhibitor A485 sensitizes prostate cancer cells to immune checkpoint inhibitors by down-regulating expression of programmed death ligand-1 (PD-L1) (153). PD-L1 is the ligand of programmed cell death 1 (PD-1) and by binding to the PD-1 receptor on the surface of T-cells immune response is repressed. Normally, PD-L1 is expressed by antigen presenting cells to prevent autoimmune 
reaction. However, cancer cells hijack PD-L1 expression to evade immune response (154). Checkpoint inhibitors blocking PD-1 or PD-L1 boost immune response and prevents immune evasion of cancer. However, PD-L1 is released exosomally by prostate cancer cells with adapted resistance to checkpoint inhibitors. The expression of PD-L1 is dependent on P300 and therefore those resistant cells can be re-sensitized by P300 inhibitor treatment.

Although P300 and CBP inhibitors are promising for the treatment of therapy resistant prostate cancer, tumors will ultimately also develop a resistance to those inhibitors. It has already been shown that mutations in Speckle-type POZ protein (SPOP) gene convey a resistance to BET inhibitors in prostate cancer. SPOP mutations often found in prostate cancer result in reduced degradation of BRD proteins and thereby induces the accumulation of BRD proteins (155). Emerging resistance to the pan bromodomain inhibitor JQ1 has also been observed. In leukemia increase in $W N T / \beta$-catenin signaling was related to bromodomain inhibitor resistance (156). In hepatocellular carcinoma resistance to JQ1 was tied to increased activity of EGFR and stabilization of MYC (157).

There have been more than two decades research on transcriptional integrators p300 and CBP in prostate cancer research. Several laboratories documented their role in regulation of agonistic and antagonistic effects of anti-androgens, proliferation, migration, and invasion. These results from basic science lead to multiple pharmacological approaches with aim to inhibit $\mathrm{p} 300 / \mathrm{CBP}$ action by targeting different domains. The effect of $\mathrm{P} 300$ and $C B P$ inhibitors was characterizing in different cancer models for a long period of time. Now there is a scientifically sound basis for use of these inhibitors in clinical studies for prostate cancer and specifically therapy resistant prostate cancer. 


\section{Abbreviation}

A488

ADT

AF1

AP $-1 / 2$

AR

ARE

AR-V7

ASAP

BET

BET

$\mathrm{BPH}$

BRD2/3/4 bromodomain-containing protein $2 / 3 / 4$

cAMP cyclic adenosine monophosphate

CBP CREB binding protein

CBP

$\mathrm{CH}$

CNA

Alexa488

androgen deprivation therapy

activation function 1

activator-protein $1 / 2$

androgen receptor

androgen response element

androgen receptor Variant 7

atypical small acinar proliferation

bromodomain and extra-terminal motif

bromo- and extra-terminal domain

benign prostatic hyperplasia

CREB binding protein

cystidin/histidin rich region

copy number abberation 


\begin{tabular}{|c|c|}
\hline CRPC & castration therapy resistant prostate cancer \\
\hline DBD & DNA binding domain \\
\hline DHT & dihydrotestosterone \\
\hline DMSO & dimethylsulfoxid \\
\hline $\mathrm{E} 2 \mathrm{~F}$ & E2 factor \\
\hline EnzaR & enzalutamide resistant sub cell line \\
\hline FACS & fluorescence-activated cell sorting \\
\hline FKBP5 & FK506 binding protein 5 \\
\hline GSEA & gene set enrichment analysis \\
\hline HAT & histon acetyl transferase \\
\hline ID1/3 & inhibitor of DNA binding $1 / 3$ \\
\hline IL6 & interleukin 6 \\
\hline IRBC & ribosome biogenesis checkpoint \\
\hline KIX & kinase-inducible domain interacting domain \\
\hline LBD & ligand binding domain \\
\hline LBD & ligand-binding domain \\
\hline LH & luteinizing hormone \\
\hline LHRH & luteinizing hormone $r$ \\
\hline
\end{tabular}

MCRPC metastatic castration therapy resistant prostate cancer 


\begin{tabular}{|c|c|}
\hline NES & nuclear export signal \\
\hline NLS & nuclear localization signal \\
\hline NTD & $\mathrm{n}$-terminal domain \\
\hline p160 & steroid receptor coactivator family \\
\hline PD-1 & programmed cell death 1 \\
\hline PD-L1 & programmed death ligand-1 \\
\hline PFN2 & profilin-2 \\
\hline PIAS & protein inhibitor of activated STAT \\
\hline PSA & prostate specific antigen \\
\hline QP & prolin rich domain \\
\hline RID & receptor interaction domain \\
\hline RNAi & RNA interference \\
\hline RP & radical prostatectomy \\
\hline RPL & ribosomal protein (of the large subunit) \\
\hline RPS & ribosomal protein (of the small subunit) \\
\hline rRNA & ribosomal RNA \\
\hline shRNA & small hairpin RNA \\
\hline SID & steroid interaction domain \\
\hline siRNA & small interfering RNA \\
\hline
\end{tabular}


SP1 specificity protein 1

SPOP speckle-type POZ protein

SRC3 steroid receptor coactivator 3

SRF serum response factor

STAT3 signal transducer and activator of transcription 3

SU2C stand up to cancer project

Tau-1 transcription activation unit 1

Tau-2 transcription activation unit 2

TGF- $\beta \quad$ transforming growth factor $\beta$

TMPRSS2 transmembrane protease, serine 2

ZEB1/2 zinc finger e-box binding homeobox $1 / 2$ 


\section{Acknowledgements}

I would like to thank Prof. Zoran Culig not only for giving me the opportunity to work on this exciting project, but also for the support and trust I received during the last years.

My gratitude also goes to Prof. Helmut Klocker, head of the laboratory of the division for experimental urology, for enabling me to conduct my work in this wonderful lab.

Further, I would like to thank the members of my thesis committee: Prof. Jakob Troppmair and Prof. Alexandra Lusser. Their advice and encouragement helped me immensely throughout my studies.

I would also like to thank all past and present members of the members of the lab for their constant technical support and for creating such a great work environment.

Finally, I would like to thank my parents for always supporting and encouraging me. 


\section{References}

(1) McNeal JE. Anatomy of the prostate and morphogenesis of BPH. Prog Clin Biol Res. 1984; 145:2753.

(2) Aaron L, Franco OE, Hayward SW. Review of Prostate Anatomy and Embryology and the Etiology of Benign Prostatic Hyperplasia. Urol Clin North Am. 2016; 43(3):279-88.

(3) Pignon J-C, Grisanzio C, Geng Y, Song J, Shivdasani RA, Signoretti S. p63-expressing cells are the stem cells of developing prostate, bladder, and colorectal epithelia. Proc Natl Acad Sci U S A. 2013; 110(20):8105-10.

(4) Toivanen R, Shen MM. Prostate organogenesis: tissue induction, hormonal regulation and cell type specification. Development. 2017; 144(8):1382-98.

(5) Parsons JK, Gage WR, Nelson WG, De Marzo AM. p63 protein expression is rare in prostate adenocarcinoma: implications for cancer diagnosis and carcinogenesis. Urology. 2001; 58(4):619-24.

(6) Goldstein AS, Huang J, Guo C, Garraway IP, Witte ON. Identification of a Cell of Origin for Human Prostate Cancer. Science. 2010; 329(5991):568-71.

(7) Chan JM, Gann PH, Giovannucci EL. Role of Diet in Prostate Cancer Development and Progression. J Clin Oncol. 2005; 23(32):8152-60.

(8) Kheirandish P, Chinegwundoh F. Ethnic differences in prostate cancer. Br J Cancer. 2011; 105(4):481-5.

(9) Spitz MR, Currier RD, Fueger JJ, Babaian RJ, Newell GR. Familial patterns of prostate cancer: a case-control analysis. J Urol. 1991; 146(5):1305-7.

(10) Ferlay J, Ervik M, Lam F, Colombet M, Mery L, Piñeros M, et al. Cancer Today [Internet]. 2020 [last access 05.05.2021]. Available from: https://gco.iarc.fr/today.

(11) Oesterling JE, Jacobsen SJ, Chute CG, Guess HA, Girman CJ, Panser LA, et al. Serum ProstateSpecific Antigen in a Community-Based Population of Healthy Men: Establishment of Age-Specific Reference Ranges. JAMA. 1993; 270(7):860-4.

(12) Gleason DF. Classification of prostatic carcinomas. Cancer Chemother Rep. 1966; 50(3):125-8. 
(13) Epstein JI, Allsbrook WCJ, Amin MB, Egevad LL, Committee atIG. The 2005 International Society of Urological Pathology (ISUP) Consensus Conference on Gleason Grading of Prostatic Carcinoma. Am J Surg Pathol. 2005; 29(9):1228-42.

(14) Epstein JI, Egevad L, Amin MB, Delahunt B, Srigley JR, Humphrey PA. The 2014 International Society of Urological Pathology (ISUP) Consensus Conference on Gleason Grading of Prostatic Carcinoma: Definition of Grading Patterns and Proposal for a New Grading System. Am J Surg Pathol. 2016; 40(2):244-52.

(15) Humphrey PA. Gleason grading and prognostic factors in carcinoma of the prostate. Mod Pathol. 2004; 17(3):292-306.

(16) Gleason DF. Histologic grading of prostate cancer: A perspective. Hum Pathol. 1992; 23(3):273-9.

(17) European Association of Urology (EAU), European Association of Nuclear Medicine (EANM), European Society for Radiotherapy and Oncology (ESTRO), European Society of Urogenital Radiology (ESUR), International Society of Geriatric Oncology (SIOG), International Society of Urologic Pathology (ISUP). Guidelines on Prostate Cancer [Online Document]. 2021 [last access 05.05.2021]. Available from: https://uroweb.org/wp-content/uploads/EAU-EANM-ESTRO_ESUR_ISUP_SIOG-Guidelines-onProstate-Cancer-2021.pdf.

(18) Crawford ED, Heidenreich A, Lawrentschuk N, Tombal B, Pompeo ACL, Mendoza-Valdes A, et al. Androgen-targeted therapy in men with prostate cancer: evolving practice and future considerations. Prostate Cancer Prostatic Dis. 2019; 22(1):24-38.

(19) Hellerstedt BA, Pienta KJ. The Current State of Hormonal Therapy for Prostate Cancer. CA Cancer J Clin. 2002; 52(3):154-79.

(20) Silva ÉD, Ferreira U, Matheus W, Faria EF, Silva GD, Saito M, et al. Goserelin versus leuprolide in the chemical castration of patients with prostate cancer. Int Urol Nephrol. 2012; 44(4):1039-44.

(21) Davey RA, Grossmann M. Androgen Receptor Structure, Function and Biology: From Bench to Bedside. Clin Biochem Rev. 2016; 37(1):3-15.

(22) Schiffer L, Arlt W, Storbeck K-H. Intracrine androgen biosynthesis, metabolism and action revisited. Mol Cell Endocrinol. 2018; 465:4-26. 
(23) Kuil CW, Berrevoets CA, Mulder E. Ligand-induced Conformational Alterations of the Androgen Receptor Analyzed by Limited Trypsinization: STUDIES ON THE MECHANISM OF ANTIANDROGEN ACTION (*). J Biol Chem. 1995; 270(46):27569-76.

(24) van Royen ME, van Cappellen WA, de Vos C, Houtsmuller AB, Trapman J. Stepwise androgen receptor dimerization. J Cell Sci. 2012; 125(Pt 8):1970-9.

(25) Denayer S, Helsen C, Thorrez L, Haelens A, Claessens F. The rules of DNA recognition by the androgen receptor. Mol Endocrinol. 2010; 24(5):898-913.

(26) Snyder PJ. Editorial: The Role of Androgens in Women. J Clin Endocrinol Metab. 2001; 86(3):1006-7.

(27) Rana K, Davey RA, Zajac JD. Human androgen deficiency: insights gained from androgen receptor knockout mouse models. Asian J Androl. 2014; 16(2):169-77.

(28) Lee D. High androgen receptor levels are predictive of decreased survival in prostate cancer. Clin Prostate Cancer. 2003; 2(1):13-4.

(29) Tan MHE, Li J, Xu HE, Melcher K, Yong E-I. Androgen receptor: structure, role in prostate cancer and drug discovery. Acta Pharmacol Sin. 2015; 36(1):3-23.

(30) Gregory CW, Johnson RT, Mohler JL, French FS, Wilson EM. Androgen Receptor Stabilization in Recurrent Prostate Cancer Is Associated with Hypersensitivity to Low Androgen. Cancer Res. 2001; 61(7):2892.

(31) Kraus S, Gioeli D, Vomastek T, Gordon V, Weber MJ. Receptor for activated C kinase 1 (RACK1) and Src regulate the tyrosine phosphorylation and function of the androgen receptor. Cancer Res. 2006; 66(22):11047-54.

(32) Wang Z, Shen H, Liang Z, Mao Y, Wang C, Xie L. The characteristics of androgen receptor splice variant 7 in the treatment of hormonal sensitive prostate cancer: a systematic review and metaanalysis. Cancer Cell Int. 2020; 20:149.

(33) Xu D, Zhan Y, Qi Y, Cao B, Bai S, Xu W, et al. Androgen Receptor Splice Variants Dimerize to Transactivate Target Genes. Cancer Res. 2015; 75(17):3663-71. 
(34) Stanbrough M, Bubley GJ, Ross K, Golub TR, Rubin MA, Penning TM, et al. Increased expression of genes converting adrenal androgens to testosterone in androgen-independent prostate cancer. Cancer Res. 2006; 66(5):2815-25.

(35) Taylor BS, Schultz N, Hieronymus H, Gopalan A, Xiao Y, Carver BS, et al. Integrative Genomic Profiling of Human Prostate Cancer. Cancer Cell. 2010; 18(1):11-22.

(36) Lin Y, Fukuchi J, Hiipakka RA, Kokontis JM, Xiang J. Up-regulation of Bcl-2 is required for the progression of prostate cancer cells from an androgen-dependent to an androgen-independent growth stage. Cell Res. 2007; 17(6):531-6.

(37) Scher HI, Fizazi K, Saad F, Taplin M-E, Sternberg CN, Miller K, et al. Increased Survival with Enzalutamide in Prostate Cancer after Chemotherapy. N Engl J Med. 2012; 367(13):1187-97.

(38) Moilanen A-M, Riikonen R, Oksala R, Ravanti L, Aho E, Wohlfahrt G, et al. Discovery of ODM-201, a new-generation androgen receptor inhibitor targeting resistance mechanisms to androgen signaling-directed prostate cancer therapies. Sci Rep. 2015; 5(1):12007.

(39) Armstrong AJ, Lin P, Tombal B, Saad F, Higano CS, Joshua AM, et al. Five-year Survival Prediction and Safety Outcomes with Enzalutamide in Men with Chemotherapy-naïve Metastatic Castrationresistant Prostate Cancer from the PREVAIL Trial. Eur Urol. 2020; 78(3):347-57.

(40) Sanford M. Enzalutamide: a review of its use in metastatic, castration-resistant prostate cancer. Drugs. 2013; 73(15):1723-32.

(41) Chen CD, Welsbie DS, Tran C, Baek SH, Chen R, Vessella R, et al. Molecular determinants of resistance to antiandrogen therapy. Nat Med. 2004; 10(1):33-9.

(42) Galletti G, Leach BI, Lam L, Tagawa ST. Mechanisms of resistance to systemic therapy in metastatic castration-resistant prostate cancer. Cancer Treat Rev. 2017; 57:16-27.

(43) Balbas MD, Evans MJ, Hosfield DJ, Wongvipat J, Arora VK, Watson PA, et al. Overcoming mutation-based resistance to antiandrogens with rational drug design. Elife. 2013; 2:e00499. doi: 10.7554/eLife.00499 [ePub 05.50.2021].

(44) Xu J, Li Q. Review of the in Vivo Functions of the p160 Steroid Receptor Coactivator Family. Mol Endocrinol. 2003; 17(9):1681-92. 
(45) Xu J, Wu RC, O'Malley BW. Normal and cancer-related functions of the p160 steroid receptor coactivator (SRC) family. Nat Rev Cancer. 2009; 9(9):615-30.

(46) Kuang SQ, Liao L, Zhang H, Lee AV, O'Malley BW, Xu J. AIB1/SRC-3 deficiency affects insulin-like growth factor I signaling pathway and suppresses v-Ha-ras-induced breast cancer initiation and progression in mice. Cancer Res. 2004; 64(5):1875-85.

(47) Mora LB, Buettner R, Seigne J, Diaz J, Ahmad N, Garcia R, et al. Constitutive Activation of Stat3 in Human Prostate Tumors and Cell Lines. Direct Inhibition of Stat3 Signaling Induces Apoptosis of Prostate Cancer Cells. Cancer Res. 2002; 62(22):6659-66.

(48) Hoefer J, Schäfer G, Klocker H, Erb HHH, Mills IG, Hengst L, et al. PIAS1 Is Increased in Human Prostate Cancer and Enhances Proliferation through Inhibition of p21. Am J Pathol. 2012; 180(5):2097-107.

(49) Dyson N, Guida P, Münger K, Harlow E. Homologous sequences in adenovirus E1A and human papillomavirus E7 proteins mediate interaction with the same set of cellular proteins. J Virol. 1992; 66(12):6893-902.

(50) Arany Z, Newsome D, Oldread E, Livingston DM, Eckner R. A family of transcriptional adaptor proteins targeted by the E1A oncoprotein. Nature. 1995; 374(6517):81-4.

(51) Kamei Y, Xu L, Heinzel T, Torchia J, Kurokawa R, Gloss B, et al. A CBP Integrator Complex Mediates Transcriptional Activation and AP-1 Inhibition by Nuclear Receptors. Cell. 1996; 85(3):40314.

(52) Gu W, Roeder RG. Activation of p53 Sequence-Specific DNA Binding by Acetylation of the p53 CTerminal Domain. Cell. 1997; 90(4):595-606.

(53) Fauquier L, Azzag K, Parra MAM, Quillien A, Boulet M, Diouf S, et al. CBP and P300 regulate distinct gene networks required for human primary myoblast differentiation and muscle integrity. Sci Rep. 2018; 8(1):12629.

(54) Kung AL, Rebel VI, Bronson RT, Ch'ng LE, Sieff CA, Livingston DM, et al. Gene dose-dependent control of hematopoiesis and hematologic tumor suppression by CBP. Genes Dev. 2000; 14(3):272-7.

(55) Goodman RH, Smolik S. CBP/p300 in cell growth, transformation, and development. Genes Dev. 2000; 14(13):1553-77. 
(56) Lundblad JR, Kwok RPS, Laurance ME, Harter ML, Goodman RH. Adenoviral ElA-associated protein p300 as a functional homologue of the transcriptional co-activator CBP. Nature. 1995; 374(6517):85-8.

(57) Karamouzis MV, Konstantinopoulos PA, Papavassiliou AG. Roles of CREB-binding protein (CBP)/p300 in respiratory epithelium tumorigenesis. Cell Res. 2007; 17(4):324-32.

(58) Yu X, Yi P, Hamilton RA, Shen H, Chen M, Foulds CE, et al. Structural Insights of Transcriptionally Active, Full-Length Androgen Receptor Coactivator Complexes. Mol Cell. 2020; 79(5):812-23.e4.

(59) Nakajima T, Uchida C, Anderson SF, Lee CG, Hurwitz J, Parvin JD, et al. RNA helicase A mediates association of CBP with RNA polymerase II. Cell. 1997; 90(6):1107-12.

(60) Eberharter A, Becker PB. Histone acetylation: a switch between repressive and permissive chromatin. Second in review series on chromatin dynamics. EMBO Rep. 2002; 3(3):224-9.

(61) Fu M, Wang C, Reutens AT, Wang J, Angeletti RH, Siconolfi-Baez L, et al. p300 and p300/cAMPresponse element-binding protein-associated factor acetylate the androgen receptor at sites governing hormone-dependent transactivation. J Biol Chem. 2000; 275(27):20853-60.

(62) Giordano A, Avantaggiati ML. p300 and CBP: Partners for life and death. J Cell Physiol. 1999; 181(2):218-30.

(63) Grossman SR. p300/CBP/p53 interaction and regulation of the p53 response. Eur J Biochem. $2001 ; 268(10): 2773-8$.

(64) Xiao XS, Cai MY, Chen JW, Guan XY, Kung HF, Zeng YX, et al. High Expression of p300 in Human Breast Cancer Correlates with Tumor Recurrence and Predicts Adverse Prognosis. Chin J Cancer Res. 2011; 23(3):201-7.

(65) Welti J, Sharp A, Brooks N, Yuan W, McNair C, Chand SN, et al. Targeting p300/CBP axis in lethal prostate cancer. Cancer Discov. 2021; 11(5):1118-37.

(66) Heemers HV, Sebo TJ, Debes JD, Regan KM, Raclaw KA, Murphy LM, et al. Androgen Deprivation Increases p300 Expression in Prostate Cancer Cells. Cancer Res. 2007; 67(7):3422-30.

(67) Debes JD, Schmidt L, Huang H, Tindall DJ. p300 Mediates Androgen-independent Transactivation of the Androgen Receptor by Interleukin 6. Cancer Res. 2002; 62(20):5632-6. 
(68) Filippakopoulos P, Qi J, Picaud S, Shen Y, Smith WB, Fedorov O, et al. Selective inhibition of BET bromodomains. Nature. 2010; 468(7327):1067-73.

(69) Yang Z, He N, Zhou Q. Brd4 Recruits P-TEFb to Chromosomes at Late Mitosis To Promote G1 Gene Expression and Cell Cycle Progression. Mol Cell Biol. 2008; 28(3):967-76.

(70) Zuber J, Shi J, Wang E, Rappaport AR, Herrmann H, Sison EA, et al. RNAi screen identifies Brd4 as a therapeutic target in acute myeloid leukaemia. Nature. 2011; 478(7370):524-8.

(71) Abbate EA, Voitenleitner C, Botchan MR. Structure of the Papillomavirus DNA-Tethering Complex E2:Brd4 and a Peptide that Ablates HPV Chromosomal Association. Mol Cell. 2006; 24(6):877-89.

(72) Spriano F, Gaudio E, Cascione L, Tarantelli C, Melle F, Motta G, et al. Antitumor activity of the dual BET and CBP/EP300 inhibitor NEO2734. Blood Adv. 2020; 4(17):4124-35.

(73) Santer FR, Höschele PP, Oh SJ, Erb HH, Bouchal J, Cavarretta IT, et al. Inhibition of the acetyltransferases p300 and CBP reveals a targetable function for p300 in the survival and invasion pathways of prostate cancer cell lines. Mol Cancer Ther. 2011; 10(9):1644-55.

(74) Lasko LM, Jakob CG, Edalji RP, Qiu W, Montgomery D, Digiammarino EL, et al. Discovery of a selective catalytic p300/CBP inhibitor that targets lineage-specific tumours. Nature. 2017; 550:128.

(75) Zucconi BE, Makofske JL, Meyers DJ, Hwang Y, Wu M, Kuroda MI, et al. Combination Targeting of the Bromodomain and Acetyltransferase Active Site of p300/CBP. Biochemistry. 2019; 58(16):213343.

(76) Aggarwal RR, Schweizer MT, Nanus DM, Pantuck AJ, Heath El, Campeau E, et al. A Phase Ib/lla Study of the Pan-BET Inhibitor ZEN-3694 in Combination with Enzalutamide in Patients with Metastatic Castration-resistant Prostate Cancer. Clin Cancer Res. 2020; 26(20):5338-47.

(77) lanculescu I, Wu D-Y, Siegmund KD, Stallcup MR. Selective Roles for cAMP Response Elementbinding Protein Binding Protein and p300 Protein as Coregulators for Androgen-regulated Gene Expression in Advanced Prostate Cancer Cells*. J Biol Chem. 2012; 287(6):4000-13.

(78) Gruber M, Ferrone L, Puhr M, Santer FR, Furlan T, Eder IE, et al. p300 is up-regulated by docetaxel and is a target in chemoresistant prostate cancer. Endocr Relat Cancer. 2020; 27(3):187-98. 
(79) Kolijn K, Verhoef El, Smid M, Böttcher R, Jenster GW, Debets R, et al. Epithelial-Mesenchymal Transition in Human Prostate Cancer Demonstrates Enhanced Immune Evasion Marked by IDO1 Expression. Cancer Res. 2018; 78(16):4671-9.

(80) Sun Y, Wang B-E, Leong KG, Yue P, Li L, Jhunjhunwala S, et al. Androgen Deprivation Causes Epithelial-Mesenchymal Transition in the Prostate: Implications for Androgen-Deprivation Therapy. Cancer Res. 2012; 72(2):527-36.

(81) Fu M, Wang C, Reutens AT, Wang J, Angeletti RH, Siconolfi-Baez L, et al. p300 and p300/cAMPresponse Element-binding Protein-associated Factor Acetylate the Androgen Receptor at Sites Governing Hormone-dependent Transactivation*. J Biol Chem. 2000; 275(27):20853-60.

(82) Debes JD, Sebo TJ, Lohse CM, Murphy LM, Haugen DA, Tindall DJ. p300 in prostate cancer proliferation and progression. Cancer Res. 2003; 63(22):7638-40.

(83) Puhr M, Hoefer J, Eigentler A, Ploner C, Handle F, Schaefer G, et al. The Glucocorticoid Receptor Is a Key Player for Prostate Cancer Cell Survival and a Target for Improved Antiandrogen Therapy. Clin Cancer Res. 2018; 24(4):927-38.

(84) Kino T, Nordeen SK, Chrousos GP. Conditional modulation of glucocorticoid receptor activities by CREB-binding protein (CBP) and p300. J Steroid Biochem Mol Biol. 1999; 70(1):15-25.

(85) Comuzzi B, Nemes C, Schmidt S, Jasarevic Z, Lodde M, Pycha A, et al. The androgen receptor coactivator CBP is up-regulated following androgen withdrawal and is highly expressed in advanced prostate cancer. J Pathol. 2004; 204(2):159-66.

(86) Comuzzi B, Lambrinidis L, Rogatsch H, Godoy-Tundidor S, Knezevic N, Krhen I, et al. The transcriptional co-activator CAMP response element-binding protein-binding protein is expressed in prostate cancer and enhances androgen- and anti-androgen-induced androgen receptor function. Am J Pathol. 2003; 162(1):233-41.

(87) Ramos YF, Hestand MS, Verlaan M, Krabbendam E, Ariyurek Y, van Galen M, et al. Genome-wide assessment of differential roles for p300 and CBP in transcription regulation. Nucleic Acids Res. 2010; 38(16):5396-408.

(88) lanculescu I, Wu D-Y, Siegmund KD, Stallcup MR. Selective Roles for cAMP Response Elementbinding Protein Binding Protein and p300 Protein as Coregulators for Androgen-regulated Gene Expression in Advanced Prostate Cancer Cells. J Biol Chem. 2012; 287(6):4000-13. 
(89) Picaud S, Fedorov O, Thanasopoulou A, Leonards K, Jones K, Meier J, et al. Generation of a Selective Small Molecule Inhibitor of the CBP/p300 Bromodomain for Leukemia Therapy. Cancer Res. 2015; 75(23):5106-19.

(90) He Z-X, Wei B-F, Zhang X, Gong Y-P, Ma L-Y, Zhao W. Current development of CBP/p300 inhibitors in the last decade. Eur J Med Chem. 2021; 209:112861.

(91) Hay DA, Fedorov O, Martin S, Singleton DC, Tallant C, Wells C, et al. Discovery and Optimization of Small-Molecule Ligands for the CBP/p300 Bromodomains. J Am Chem Soc. 2014; 136(26):9308-19.

(92) Popp TA, Tallant C, Rogers C, Fedorov O, Brennan PE, Müller S, et al. Development of Selective CBP/P300 Benzoxazepine Bromodomain Inhibitors. J Med Chem. 2016; 59(19):8889-912.

(93) Taylor AM, Côté A, Hewitt MC, Pastor R, Leblanc Y, Nasveschuk CG, et al. Fragment-Based Discovery of a Selective and Cell-Active Benzodiazepinone CBP/EP300 Bromodomain Inhibitor (CPI637). ACS Med Chem Lett. 2016; 7(5):531-6.

(94) Bowers EM, Yan G, Mukherjee C, Orry A, Wang L, Holbert MA, et al. Virtual ligand screening of the $\mathrm{p} 300 / \mathrm{CBP}$ histone acetyltransferase: identification of a selective small molecule inhibitor. Chem Biol. 2010; 17(5):471-82.

(95) Liu X, Wang L, Zhao K, Thompson PR, Hwang Y, Marmorstein R, et al. The structural basis of protein acetylation by the p300/CBP transcriptional coactivator. Nature. 2008; 451(7180):846-50.

(96) Lasko LM, Jakob CG, Edalji RP, Qiu W, Montgomery D, Digiammarino EL, et al. Discovery of a selective catalytic p300/CBP inhibitor that targets lineage-specific tumours. Nature. 2017; 550(7674):128-32.

(97) Zhong J, Ding L, Bohrer LR, Pan Y, Liu P, Zhang J, et al. p300 Acetyltransferase Regulates Androgen Receptor Degradation and PTEN-Deficient Prostate Tumorigenesis. Cancer Res. 2014; 74(6):1870-80.

(98) Lee SO, Chun JY, Nadiminty N, Lou W, Feng S, Gao AC. Interleukin-4 activates androgen receptor through CBP/p300. Prostate. 2009; 69(2):126-32.

(99) Davis ID, Martin AJ, Stockler MR, Begbie S, Chi KN, Chowdhury S, et al. Enzalutamide with Standard First-Line Therapy in Metastatic Prostate Cancer. N Engl J Med. 2019; 381(2):121-31. 
(100) Hoefer J, Akbor M, Handle F, Ofer P, Puhr M, Parson W, et al. Critical role of androgen receptor level in prostate cancer cell resistance to new generation antiandrogen enzalutamide. Oncotarget. 2016; 7(37):59781-94.

(101) Abida W, Cyrta J, Heller G, Prandi D, Armenia J, Coleman I, et al. Genomic correlates of clinical outcome in advanced prostate cancer. Proc Natl Acad Sci U S A. 2019; 116(23):11428-36.

(102) Dang CV, O'Donnell KA, Zeller KI, Nguyen T, Osthus RC, Li F. The c-Myc target gene network. Semin Cancer Biol. 2006; 16(4):253-64.

(103) Buttyan R, Sawczuk IS, Benson MC, Siegal JD, Olsson CA. Enhanced expression of the c-myc protooncogene in high-grade human prostate cancers. Prostate. 1987; 11(4):327-37.

(104) Gil J, Kerai P, Lleonart M, Bernard D, Cigudosa JC, Peters G, et al. Immortalization of Primary Human Prostate Epithelial Cells by c-Myc. Cancer Res. 2005; 65(6):2179-85.

(105) Bai S, Cao S, Jin L, Kobelski M, Schouest B, Wang X, et al. A positive role of c-Myc in regulating androgen receptor and its splice variants in prostate cancer. Oncogene. 2019; 38(25):4977-89.

(106) Li C, Lanman NA, Kong Y, He D, Mao F, Farah E, et al. Inhibition of the erythropoietin-producing receptor EPHB4 antagonizes androgen receptor overexpression and reduces enzalutamide resistance. J Biol Chem. 2020; 295(16):5470-83.

(107) Kaltz-Wittmer C, Klenk U, Glaessgen A, Aust DE, Diebold J, Löhrs U, et al. FISH Analysis of Gene Aberrations (MYC, CCND1, ERBB2, RB, and AR) in Advanced Prostatic Carcinomas Before and After Androgen Deprivation Therapy. Lab Invest. 2000; 80(9):1455-64.

(108) Bernard D, Pourtier-Manzanedo A, Gil J, Beach DH. Myc confers androgen-independent prostate cancer cell growth. J Clin Invest. 2003; 112(11):1724-31.

(109) Handle F, Prekovic S, Helsen C, Van den Broeck T, Smeets E, Moris L, et al. Drivers of AR indifferent anti-androgen resistance in prostate cancer cells. Sci Rep. 2019; 9(1):13786.

(110) Vervoorts J, Luscher-Firzlaff JM, Rottmann S, Lilischkis R, Walsemann G, Dohmann K, et al. Stimulation of c-MYC transcriptional activity and acetylation by recruitment of the cofactor CBP. EMBO Rep. 2003; 4(5):484-90. 
(111) Faiola F, Liu X, Lo S, Pan S, Zhang K, Lymar E, et al. Dual regulation of c-Myc by p300 via acetylation-dependent control of Myc protein turnover and coactivation of Myc-induced transcription. Mol Cell Biol. 2005; 25(23):10220-34.

(112) Garcia-Carpizo V, Ruiz-Llorente S, Sarmentero J, Graña-Castro O, Pisano DG, Barrero MJ. CREBBP/EP300 bromodomains are critical to sustain the GATA1/MYC regulatory axis in proliferation. Epigenetics Chromatin. 2018; 11(1):30.

(113) Destefanis F, Manara V, Bellosta P. Myc as a Regulator of Ribosome Biogenesis and Cell Competition: A Link to Cancer. Int J Mol Sci. 2020; 21(11):4037.

(114) Penzo M, Montanaro L, Treré D, Derenzini M. The Ribosome Biogenesis-Cancer Connection. Cells. 2019; 8(1):55.

(115) Barna M, Pusic A, Zollo O, Costa M, Kondrashov N, Rego E, et al. Suppression of Myc oncogenic activity by ribosomal protein haploinsufficiency. Nature. 2008; 456(7224):971-5.

(116) Pestov DG, Strezoska Ž, Lau LF. Evidence of p53-Dependent Cross-Talk between Ribosome Biogenesis and the Cell Cycle: Effects of Nucleolar Protein Bop1 on G1S Transition. Mol Cell Biol. $2001 ; 21(13): 4246-55$.

(117) van Bokhoven A, Varella-Garcia M, Korch C, Johannes WU, Smith EE, Miller HL, et al. Molecular characterization of human prostate carcinoma cell lines. Prostate. 2003; 57(3):205-25.

(118) Bee A, Ke Y, Forootan S, Lin K, Beesley C, Forrest SE, et al. Ribosomal protein I19 is a prognostic marker for human prostate cancer. Clin Cancer Res. 2006; 12 (7 Pt 1):2061-5.

(119) Bee A, Brewer D, Beesley C, Dodson A, Forootan S, Dickinson T, et al. siRNA Knockdown of Ribosomal Protein Gene RPL19 Abrogates the Aggressive Phenotype of Human Prostate Cancer. PLoS One. 2011; 6(7):e22672. https://doi.org/10.1371/journal.pone.0022672 [05.05.2021 ePub].

(120) Ebright RY, Lee S, Wittner BS, Niederhoffer KL, Nicholson BT, Bardia A, et al. Deregulation of ribosomal protein expression and translation promotes breast cancer metastasis. Science. 2020; 367(6485):1468-73.

(121) Shi Z, Fujii K, Kovary KM, Genuth NR, Röst HL, Teruel MN, et al. Heterogeneous Ribosomes Preferentially Translate Distinct Subpools of mRNAs Genome-wide. Mol Cell. 2017; 67(1):71-83.e7. 
(122) Shi Z, Fujii K, Kovary KM, Genuth NR, Röst HL, Teruel MN, et al. Heterogeneous Ribosomes Preferentially Translate Distinct Subpools of mRNAs Genome-wide. Mol Cell. 2017; 67(1):71-83.e7.

(123) Kondrashov N, Pusic A, Stumpf CR, Shimizu K, Hsieh AC, Ishijima J, et al. Ribosome-mediated specificity in Hox mRNA translation and vertebrate tissue patterning. Cell. 2011; 145(3):383-97.

(124) Zhou C, Zang D, Jin Y, Wu H, Liu Z, Du J, et al. Mutation in ribosomal protein L21 underlies hereditary hypotrichosis simplex. Hum Mutat. 2011; 32(7):710-4.

(125) De Keersmaecker K, Atak ZK, Li N, Vicente C, Patchett S, Girardi T, et al. Exome sequencing identifies mutation in CNOT3 and ribosomal genes RPL5 and RPL10 in T-cell acute lymphoblastic leukemia. Nat Genet. 2013; 45(2):186-90.

(126) Hald $\varnothing \mathrm{H}$, Olsen L, Gallo-Oller G, Elfman LHM, Løkke C, Kogner P, et al. Inhibitors of ribosome biogenesis repress the growth of MYCN-amplified neuroblastoma. Oncogene. 2019; 38(15):2800-13.

(127) Devlin JR, Hannan KM, Hein N, Cullinane C, Kusnadi E, Ng PY, et al. Combination Therapy Targeting Ribosome Biogenesis and mRNA Translation Synergistically Extends Survival in MYC-Driven Lymphoma. Cancer Discov. 2016; 6(1):59-70.

(128) Pelletier J, Thomas G, Volarević S. Ribosome biogenesis in cancer: new players and therapeutic avenues. Nat Rev Cancer. 2018; 18(1):51-63.

(129) Yang D, Okamoto K. Structural insights into G-quadruplexes: towards new anticancer drugs. Future Med Chem. 2010; 2(4):619-46.

(130) Drygin D, Lin A, Bliesath J, Ho CB, O'Brien SE, Proffitt C, et al. Targeting RNA Polymerase I with an Oral Small Molecule CX-5461 Inhibits Ribosomal RNA Synthesis and Solid Tumor Growth. Cancer Res. 2011; 71(4):1418-30.

(131) Drygin D, Siddiqui-Jain A, O'Brien S, Schwaebe M, Lin A, Bliesath J, et al. Anticancer activity of CX-3543: a direct inhibitor of rRNA biogenesis. Cancer Res. 2009; 69(19):7653-61.

(132) El Gammal AT, Brüchmann M, Zustin J, Isbarn H, Hellwinkel OJC, Köllermann J, et al. Chromosome 8p Deletions and 8q Gains are Associated with Tumor Progression and Poor Prognosis in Prostate Cancer. Clin Cancer Res. 2010; 16(1):56-64. 
(133) Mehrazin R, Dulaimi E, Uzzo RG, Devarjan K, Pei J, Smaldone MC, et al. The correlation between gain of chromosome $8 q$ and survival in patients with clear and papillary renal cell carcinoma. Ther Adv Urol. 2018; 10(1):3-10

(134) de Krijger RR, Claessen SMH, van der Ham F, van Unnik AJM, Hulsbergen-van de Kaa CA, van Leuven L, et al. Gain of chromosome 8q is a frequent finding in pleuropulmonary blastoma. Mod Pathol. 2007; 20(11):1191-9.

(135) Kang JU. Chromosome 8q as the most frequent target for amplification in early gastric carcinoma. Oncol Lett. 2014; 7(4):1139-43.

(136) Shi Y, Wu H, Zhang M, Ding L, Meng F, Fan X. Expression of the epithelial-mesenchymal transition-related proteins and their clinical significance in lung adenocarcinoma. Diagn Pathol. 2013; 8:89.

(137) Arumugam T, Ramachandran V, Fournier KF, Wang H, Marquis L, Abbruzzese JL, et al. Epithelial to Mesenchymal Transition Contributes to Drug Resistance in Pancreatic Cancer. Cancer Res. 2009; 69(14):5820-8.

(138) Puhr M, Hoefer J, Schäfer G, Erb HHH, Oh SJ, Klocker H, et al. Epithelial-to-Mesenchymal Transition Leads to Docetaxel Resistance in Prostate Cancer and Is Mediated by Reduced Expression of miR-200c and miR-205. Am J Pathol. 2012; 181(6):2188-201.

(139) Quintayo MA, Munro AF, Thomas J, Kunkler IH, Jack W, Kerr GR, et al. GSK3 $\beta$ and cyclin D1 expression predicts outcome in early breast cancer patients. Breast Cancer Res Treat. 2012; 136(1):161-8

(140) Qie S, Diehl JA. Cyclin D1, cancer progression, and opportunities in cancer treatment. J Mol Med (Berl). 2016; 94(12):1313-26.

(141) Takebe N, Harris PJ, Warren RQ, Ivy SP. Targeting cancer stem cells by inhibiting Wnt, Notch, and Hedgehog pathways. Nat Rev Clin Oncol. 2011; 8(2):97-106.

(142) Li X, Placencio V, Iturregui JM, Uwamariya C, Sharif-Afshar AR, Koyama T, et al. Prostate tumor progression is mediated by a paracrine TGF-beta/Wnt3a signaling axis. Oncogene. 2008; 27(56):711830. 
(143) Beltran H, Rickman DS, Park K, Chae SS, Sboner A, MacDonald TY, et al. Molecular characterization of neuroendocrine prostate cancer and identification of new drug targets. Cancer Discov. 2011; 1(6):487-95.

(144) Pan W, Zhang Z, Kimball H, Qu F, Berlind K, Stopsack KH, et al. Abiraterone Acetate Induces CREB1 Phosphorylation and Enhances the Function of the CBP-p300 Complex, Leading to Resistance in Prostate Cancer Cells. Clin Cancer Res. 2021; 27(7):2087-99.

(145) Yuan LW, Gambee JE. Histone acetylation by p300 is involved in CREB-mediated transcription on chromatin. Biochim Biophys Acta. 2001; 1541(3):161-9.

(146) He Y, Wei T, Ye Z, Orme JJ, Lin D, Sheng H, et al. A noncanonical AR addiction drives enzalutamide resistance in prostate cancer. Nat Commun. 2021; 12(1):1521.

(147) Garcia-Carpizo V, Ruiz-Llorente S, Sarmentero J, Gonzalez-Corpas A, Barrero MJ. CREBBP/EP300 bromodomain inhibition affects the proliferation of AR positive breast cancer cell lines. Mol Cancer Res. $2019 ; 17(3): 720-30$.

(148) Xu S, Fan L, Jeon H-Y, Zhang F, Cui X, Mickle MB, et al. p300-Mediated Acetylation of Histone Demethylase JMJD1A Prevents Its Degradation by Ubiquitin Ligase STUB1 and Enhances Its Activity in Prostate Cancer. Cancer Res. 2020; 80(15):3074-87.

(149) Lasko LM, Jakob CG, Edalji RP, Qiu W, Montgomery D, Digiammarino EL, et al. Discovery of a selective catalytic p300/CBP inhibitor that targets lineage-specific tumours. Nature. 2017; 550(7674):128-32.

(150) Romero FA, Murray J, Lai KW, Tsui V, Albrecht BK, An L, et al. GNE-781, A Highly Advanced Potent and Selective Bromodomain Inhibitor of Cyclic Adenosine Monophosphate Response Element Binding Protein, Binding Protein (CBP). J Med Chem. 2017; 60(22):9162-83.

(151) Giles F, Witcher M, Brown B. 429P - NEO2734: A novel potent oral dual BET and P300/CBP inhibitor. Ann Oncol. 2018; 29(8):140-1.

(152) Spriano F, Stathis A, Bertoni F. Targeting BET bromodomain proteins in cancer: The example of lymphomas. Pharmacol Ther. 2020; 215:107631. 
(153) Liu J, He D, Cheng L, Huang C, Zhang Y, Rao X, et al. p300/CBP inhibition enhances the efficacy of programmed death-ligand 1 blockade treatment in prostate cancer. Oncogene. 2020; 39(19):393951.

(154) Chen L, Han X. Anti-PD-1/PD-L1 therapy of human cancer: past, present, and future. J Clin Invest. 2015; 125(9):3384-91.

(155) Zhang P, Wang D, Zhao Y, Ren S, Gao K, Ye Z, et al. Intrinsic BET inhibitor resistance in SPOPmutated prostate cancer is mediated by BET protein stabilization and AKT-mTORC1 activation. Nat Med. 2017; 23(9):1055-62.

(156) Fong CY, Gilan O, Lam EY, Rubin AF, Ftouni S, Tyler D, et al. BET inhibitor resistance emerges from leukaemia stem cells. Nature. 2015; 525(7570):538-42.

(157) Yin Y, Sun M, Zhan X, Wu C, Geng P, Sun X, et al. EGFR signaling confers resistance to BET inhibition in hepatocellular carcinoma through stabilizing oncogenic MYC. J Exp Clin Cancer Res. 2019; 38(1):83. 


\section{Appendix}

MYC-Mediated Ribosomal Gene Expression Sensitizes Enzalutamideresistant Prostate Cancer Cells to EP300/CREBBP Inhibitors

Am J Pathol. 2021 Mar 8;S0002-9440(21)00084-5.

Ahead of print.

doi: 10.1016/j.ajpath.2021.02.017.

This is an open access article distributed under the terms of the Creative Commons CC-BY license, which permits unrestricted use, distribution, and reproduction in any medium, provided the original work is properly cited. 
Q6 Prostate cancer (PCa) is one of the most commonly diagnosed types of cancer, especially in industrialized nations. Therapy of localized PCa is mostly curative. For recurrent $\mathrm{PCa}$, androgen deprivation therapy via chemical or surgical castration remains the current standard-of-care treatment. ${ }^{1}$ Although initially effective, the development of castration-resistant PCa is nearly always inevitable. For these patients, blockade of the androgen receptor (AR) is used, commonly with the antiandrogen enzalutamide. Enzalutamide not only inhibits ligand binding to the $\mathrm{AR}$, but it also reduces the translocation of the AR to the nucleus as well as DNA binding. ${ }^{2}$ Nevertheless, similar to androgen deprivation therapy, enzalutamide is only effective for a short time. Subsequent therapeutic options for enzalutamide-resistant PCa are limited.
Several molecular mechanisms leading to enzalutamide resistance have already been described, including elevated activation of the AR either through overexpression or mutation, expression of AR splice variants, amplification of AR co-activators, enhanced functional activity of glucocorticoid receptor, or MYC overexpression. ${ }^{3}$ The histone acetyl transferase (HAT) EP300 (P300) and its paralogue CREBBP (CBP) have been shown to be involved in several signaling pathways associated with therapy resistance. ${ }^{4}$

Supported by the Austrian Science Fund FWF grant W1101-B12 to Z.C. Q2 Q3 and P31122 to N.S.

Disclosures: None declared.

Copyright (C 2021 American Society for Investigative Pathology. Published by Elsevier Inc. 
EP300 and CREBBP are multifunctional proteins containing a highly conserved core region that consists of a HAT domain and a bromodomain (BD). The HAT domain catalyzes the transfer of acetyl groups to lysine residues in histones, thereby relaxing the chromatin. However, many other proteins can also be acetylated, including EP300 and CREBBP themselves. ${ }^{5}$ The BD can enhance the efficacy of the HAT domain by binding to acetylated lysines, thus enabling acetylation of several lysines of a target protein., EP300 and CREBBP are well-known AR coactivators that also acetylate the AR, thus enhancing its activity. ${ }^{8}$ Furthermore, EP300 and CREBBP have been attributed to regulating MYC signaling either via direct interaction or modulation of histones. ${ }^{9}$ The importance of EP300/ CREBBP in therapy resistance is further underscored by previous studies showing that they exert oncogenic functions in $\mathrm{PCa} .{ }^{10}$ These two coactivators are up-regulated during androgen ablation and implicated in nonsteroidal AR activation. Furthermore, EP300 is associated with regulating cancer hallmarks, including proliferation, apoptosis, migration, and invasion. ${ }^{4,11,12}$ Our group recently identified EP300 as a valid target in a chemotherapeutic setting. ${ }^{13}$ Consistently, small molecular inhibitors targeting EP300, its paralogue CREBBP, or related proteins of the BRD family have shown promising results in leukemia, colorectal cancer, melanoma, and PCa. ${ }^{14-16}$

Based on the involvement of EP300/CREBBP in multiple pathways associated with castration resistance, we hypothesized that targeting the HAT or BD domains of EP300 and CREBBP may regulate cellular processes in enzalutamideresistant PCa cells. The goal of the current study, therefore, was to investigate the potential of EP300/CREBBP inhibitors in enzalutamide-sensitive and -resistant PCa models and characterize the cellular response. We observed that enzalutamide-resistant cells are highly sensitive to EP300/ CREBBP inhibitors. Intriguingly, EP300/CREBBP inhibitors not only antagonized AR function but also downregulated the expression of ribosomal proteins. Consistently, ribosomal proteins were found to be overexpressed in enzalutamide-resistant cell models as well as in a subgroup of castration-resistant PCa patient samples.

\section{Materials and Methods}

\section{Cell Culture}

[T1] LNCaP (Table 1) and PC3 cells were purchased from ATCC (Gaithersburg, MD), and DuCaP cells (Table 1) were a gift from Dr. J.A. Schalken, Nijmegen, the Netherlands. ${ }^{17-20}$ DuCaP cells have previously been established by xenografting followed by plating of metastatic tissue from the dura mater of a patient with androgen deprivation therapyresistant PCa into a SCID mouse. ${ }^{19}$ This cell line shows a near triploid karyotype with complex structural rearrangements, loss of heterozygosity of TP53, and a high amplification of wild-type AR gene, as well as expression of AR variant 7 , which is in contrast to LNCaP cells. ${ }^{17}$

Enzalutamide-resistant cells were generated by chronic treatment with increasing concentrations of enzalutamide and have been described previously. ${ }^{21,22}$ Enzalutamideresistant cells are denoted with the suffix EnzaR. All cells were cultured in RPMI 1640 (PAN-Biotech, Aidenbach, Germany) with $10 \%$ fetal calf serum (PAN-Biotech), $1 \%$ penicillin/streptomycin (Lonza, Basel, Switzerland), and 1\% GlutaMAX (Thermo Fisher Scientific, Waltham, MA). Enzalutamide-resistant cells were additionally supplied with $8 \mu \mathrm{M}$ (DuCaP EnzaR) and $5 \mu \mathrm{M}$ (LNCaP EnzaR) enzalutamide. The identity of all cell lines was confirmed by short tandem repeat analysis.

For knockdown of MYC, four different pooled siRNA constructs (ON-TARGETplus Human MYC siRNA SMARTPool, THP, Vienna, Austria) or control (ONTARGETplus siControl SMARTPool, THP) were used. For transfection, Lipofectamine RNAiMAX (Thermo Fisher Scientific) was used according to the manufacturer's instructions. After 3 days, cells were lysed in radioimmunoprecipitation assay buffer, and protein was blotted.

\section{Western Blot}

A total of $20 \mu \mathrm{g}$ protein per sample was loaded onto either 3 to $8 \%$ Tris-Acetate for EP300/CREBBP detection or 4 to $12 \%$ Bis-Tris NuPAGE protein gels (Thermo Fisher Scientific) for electrophoretic separation. Gels were then blotted onto a $0.2 \mu \mathrm{m}$ Amersham Protran Nitrocellulose membrane (Sigma, St. Louis, MO). Membranes were stained by using Revert 700 Total Protein Stain (LI-COR Biosciences, Lincoln, NE) to quantify total protein. Membranes were incubated in Starting Blocking Buffer (Thermo Fisher Scientific) for 1 hour at room temperature before overnight incubation with a primary antibody at $4{ }^{\circ} \mathrm{C}$. After washing with Tris-buffered saline containing $0.1 \%$ Tween20 (hereafter termed TBST), membranes were incubated with IRDye Goat anti-Rabbit/Mouse IgG Secondary Antibody (LI-COR Biosciences) for 45 minutes. The Odyssey imaging System (LI-COR Biosciences) was used to scan membranes after washing with TBST and the Image Studio software (version 5.2, LI-COR Biosciences) to quantify protein amounts. The same blots were probed repeatedly. The following antibodies were used at the indicated dilutions: CREBBP (7389S, Cell Signaling Technology, Danvers, MA; 1:2000), EP300 (ab10485, Abcam, Cambridge, UK; 1:1000), FKBP5/FKBP51(A301-430A, Bethyl, Montgomery, TX; 1:2000), alpha tubulin (sc-5286, Santa Cruz Laboratories, Dallas, TX; 1:2000), MYC (D84C12, Cell Signaling; 1:1000), RPL29 (AP20452c-ev, ABcepta, San Diego, CA; 1:500), RPL36 (ELA-E-AB-32803 to 60, Elabscience Biotechnology, Houston, TX; 1:500), and glyceraldehyde-3-phosphate dehydrogenase (ABS16, Millipore, Burlington, MA; 1:5000). 
Table 1 Overview of Cell Lines Used

\begin{tabular}{llll}
\cline { 2 - 4 } Q18 & LNCaP & DuCaP & Ref. \\
\cline { 2 - 4 } & $\begin{array}{c}\text { Androgen } \\
\text { sensitive } \\
\text { Origin of metastatic }\end{array}$ & ADT resistant & 17,18 \\
$\quad$ tissue & Lymph node & Dura mater & 18,19 \\
TP53 & No & Heterozygous loss & 17 \\
TMPRSS2-ERG fusion & Yes & 20 \\
AR & T877A & Wt, gene & 17 \\
AR-V7 expression & No & Yes & 17 \\
\hline
\end{tabular}

ADT, androgen deprivation therapy.

\section{Viability Assay}

Parental and enzalutamide-resistant $\mathrm{DuCaP}$ and $\mathrm{LNCaP}$ cells were seeded in a 384-well plate (Corning, Corning, NY) in triplicate at 200 and 2000 cells per well, respectively, and treated with different concentrations of the inhibitors C646 (Sigma), I-CBP112 (Tocris, Bristol, UK), CPI637 (MedChem Express, Monmouth Junction, NJ), CX5461 (MedChem Express), or the solvent dimethyl sulfoxide (Sigma). RealTime-Glo MT Cell viability assay (Promega, Madison, WI) was used as described by the manufacturer. The Cytation5 (BioTek, Winooski, VT) plate reader equipped with a carbon dioxide incubation chamber and heated to $37^{\circ} \mathrm{C}$ was used to quantify viability via Q8 luminescence over 72 hours. Dose-response curves were generated with GraphPad Prism 8 (GraphPad Software Inc., La Jolla, CA) and 50\% inhibitory concentrations were compared by using the sum-of-square-F-test.

\section{Quantitative Real-Time PCR}

Quantitative real-time PCR was performed as previously described. ${ }^{23}$ Total RNA was isolated by using the EXTRACTME RNA Isolation kit (Blirt, Zgierz, Poland) according to the manufacturer's instructions. LUNAScript kit (New England Biolabs, Ipswich, MA) was used to transcribe $250 \mathrm{ng}$ RNA into cDNA. For quantitative real- ${ }^{110}$ time PCR, cDNA was mixed with LUNA Universal Probe RT-qPCR Master Mix (New England Biolabs) and assessed in duplicate on a 7500 Fast Real-Time PCR System (Applied Biosystems, Foster City, CA). Geometric mean of the CT values of reference genes HMBS, HPRT1, and TBP were used for normalization. FKBP5, PSA, and TMPRSS2 were used as targets (Table 2).

\section{AR Reporter Gene Assay}

Parental and enzalutamide-resistant $\mathrm{DuCaP}$ and $\mathrm{LNCaP}$ cells were seeded in 96-well plates at $2 \times 10^{4}$ and $1 \times 10^{4}$ cells per well, respectively. Cells were transfected by using $\mathrm{x}$-tremeGENE HP (Roche, Basel, Switzerland) with $50 \mathrm{ng}$ pGL4.53 (Promega) encoding firefly luciferase under the control of a constitutive promoter (PGK) for normalization and $50 \mathrm{ng}$ of pGL4.70 (Promega) encoding NanoLuc under the control of a TATA-box preceded by two androgen response elements. After 5 days, cells were treated with inhibitors or R1881. AR activity was measured after 24 hours by using the Nano-Glo Dual-Luciferase Reporter Assay System (Promega) on a Cytation5 microplate reader (BioTek).

\section{RNA-Sequencing}

Parental and enzalutamide-resistant $\mathrm{DuCaP}$ and $\mathrm{LNCaP}$ cells were seeded in 6 -well plates at $8 \times 10^{5}$ and $6 \times 10^{5}$ cells per well. The following day, DuCaP cells were treated with $8 \mu \mathrm{M}$ enzalutamide, $10 \mu \mathrm{M}$ C646, $10 \mu \mathrm{M}$ I-CBP112, or dimethyl sulfoxide equivalent for 24 hours in triplicate. Total RNA was extracted by using the EXTRACTME RNA Isolation kit (Blirt) according to the manufacturer's instructions. Quality control by Bioanalyzer, poly(A) enrichment, cDNA synthesis, library preparation, Illumina sequencing, and trimming were performed at Microsynth AG (Balgach, Switzerland). One replicate of DuCaP cells

Table 2 Primer Pairs Used for RT-qPCR

\begin{tabular}{|c|c|c|c|}
\hline & Gene & Sequence/Catalog no. & Manufacturer \\
\hline Housekeeping genes & $\begin{array}{l}\text { TBP } \\
\text { HPRT }\end{array}$ & $\begin{array}{l}\text { Forward: 5'-CACGAACCACGGCACTGATT-3' } \\
\text { Reverse: 5'-TTTTCTTGCTGCCAGTCTGGAC-3' } \\
\text { Probe: 5'- TCTTCACTCTTGGCTCCTGTGCACA-3'-TAMRA } \\
\text { Forward: 5'- GCTTTCCTTGGTCAGGCAGTA-3' } \\
\text { Reverse: } 5^{\prime} \text {-GTCTGGCTTATATCCAACACTTCGT-3' } \\
\text { Probe: 5'-CAAGGTCGCAAGCTTGCTGGTGAAAAGGA-3'-TAMRA }\end{array}$ & Microsynth AG \\
\hline & HMBS & TaqMan Gene Expression Assay: Hs00609297_m1 & Thermo Fisher Scientific \\
\hline Target genes & $\begin{array}{l}\text { FKBP5 } \\
\text { TMPRSS2 } \\
\text { PSA }\end{array}$ & $\begin{array}{l}\text { TaqMan Gene Expression Assay: Hs01561006_m1 } \\
\text { TaqMan Gene Expression Assay: Hs01120965_m1 } \\
\text { Forward: 5'-GTCTGCGGGGGTTCTG-3' } \\
\text { Reverse: 5'-TGCCGACCAGCAAGATC-3' } \\
\text { Probe: 5'-CACAGCTGCCCACTGCATCAGGA-3'-TAMRA }\end{array}$ & $\begin{array}{l}\text { Thermo Fisher Scientific } \\
\text { Thermo Fisher Scientific } \\
\text { Microsynth AG }\end{array}$ \\
\hline
\end{tabular}

RT-qPCR, quantitative real-time PCR. 
was removed for quality analysis. Alignment, count tables, and differential gene expression analysis were performed by using the online platform usegalaxy.org (last accessed June 2020). ${ }^{24}$ Sequences were aligned to HG19 by using HiSat2 version 2.1.0. ${ }^{25}$ HTSeq version 0.61 was used to generate count tables. ${ }^{26}$ Differential gene expression analysis was performed by using DESeq2 version 2.11. ${ }^{27}$ ClusterProfiler version 3.16.0 was used to screen up- or down-regulated genes for overrepresented Gene Ontology version 3.11.4 annotations from Cellular Component. ${ }^{28,29} \mathrm{Up}$ - and downregulation of gene signatures in DESeq2 data was quantified by using GSEA (Broad Institute, Cambridge, MA). ${ }^{30}$ All tools used standard settings. Data are deposited in Gene Expression Omnibus (www.ncbi.nlm.nih.gov/geo/ GSE163240; accession number GSE163240).

\section{Bioinformatics/Patient Data}

The mRNA expression (FPKM, polyA) and copy number aberration data for the Stand Up To Cancer data set were downloaded from cBioPortal (dataset version from February $13,2020)$. We did not observe any difference compared with the data set version created in July $2020 .{ }^{31}$ Gene set activity was calculated with Gene Set Variation Analysis version 1.36.2 in $\mathrm{R}$ statistical software version 4.0.1 ( $\mathrm{R}$ Foundation for Statistical Computing, Vienna, Austria). The r score and $P$ value were calculated by using Pearson correlation. The heatmaps were generated with ComplexHeatmap (version 2.4.3).

\section{Trans-Well Migration}

Parental $\mathrm{LNCaP}$ and $\mathrm{LNCaP}$ EnzaR cells were treated for 24 hours as described. Cells were harvested and $1 \times 10^{5}$ cells per well were re-treated in serum-free medium and seeded in duplicate into 24-well FluoroBlok inserts with an $8 \mu \mathrm{m}$ pore size (Corning). Growth medium containing $10 \%$ fetal calf serum was used as a chemoattractant in the lower chamber. After 48 hours, cells were stained for 1 hour with $4 \mu \mathrm{mol} / \mathrm{L}$ Calcein AM (Sigma) and dissolved in Hanks' Balanced Salt Solution (Lonza) with $0.1 \%$ bovine serum albumin (Sigma). Staining solution was removed and Hanks' Balanced Salt Solution with $0.1 \%$ bovine serum albumin added. Calcein AM staining was quantified on a Cytation5 microplate reader (BioTek) and representative pictures taken on a JuLI smart fluorescent cell analyzer (Science Services, Munich, Germany). The percentage of migrated cells was calculated relative to the total number of cells seeded in parallel without inserts but with the same treatment to account for reduced cell number in CX5461-treated wells.

\section{Statistical Analysis}

For statistical comparison of independent replicates from Western blot, reporter gene assay, quantitative real-time
PCR, and Boyden-chamber assay replicates were normalized to the average signal intensity. One-way or two-way analysis of variance with Dunnett's or Tukey's multiple comparisons test was used unless stated otherwise. $P$ values of $<0.05,<0.01$, and $<0.001$ are denoted in figures by $*$, $* *$, and $* * *$, respectively.

\section{Results}

Enzalutamide-resistant Cells Are More Sensitive to EP300/CREBBP Inhibitors than Their Parental Counterparts

To investigate the therapeutic potential of EP300/CREBBP inhibition in the setting of enzalutamide resistance, we used previously established enzalutamide-resistant $\mathrm{DuCaP}$ and LNCaP cell lines (henceforth denoted as DuCaP EnzaR and LNCaP EnzaR, respectively). Protein expression of EP300/ CREBBP was clearly detectable, with no significant differences between enzalutamide-sensitive or -resistant cell lines (Figure 1A). We therefore tested the growth inhibitory effect of two compounds directed against different domains of EP300 and CREBBP in dose-response proliferation assays. C646 is an inhibitor of the HAT domain, and ICBP112 targets the BD. ${ }^{32,33}$ Specific inhibitors of either EP300 or CREBBP are not available due to high sequence similarities.

Viability was strongly reduced in all cell lines upon treatment with the EP300/CREBBP inhibitors, as determined by a real-time cell viability assay (Figure 1B). Interestingly, both enzalutamide-resistant cell lines were more sensitive to C646 inhibitor treatment compared with their nonresistant parental counterparts (Figure 1, C and D). After treatment with the BD inhibitors, only the LNCaP EnzaR cells exhibited a significantly greater sensitivity, compared with parental LNCaP cells (Figure 1, $\mathrm{C}$ and D). Because EP300/CREBBP are important AR cofactors, we were interested if higher sensitivity of enzalutamide-resistant cells might be due to effects on AR activity. To this end, we first measured the basal AR activity and androgen dependency in the cell lines using a dual luciferase AR reporter gene assay (Supplemental Figure S1A). Interestingly, LNCaP EnzaR cells exhibited a strong up-regulation of AR activity compared with that of the parental cells, which was independent of R1881 treatment. In contrast, DuCaP EnzaR cells showed the same response as the parental cells, which suggests that the two enzalutamide-resistant cell lines have different mechanisms of resistance. Importantly, AR activity was reduced upon inhibitor treatment in all cell lines, with little differences between the inhibitors (Figure 1E). These findings were further validated by assessment of AR target gene expression, including KLK3 (PSA), FKBP5, and TMPRSS2, which also exhibited down-regulation (Supplemental Figure S1B). Similar results were also observed at the protein level for FKBP5 (Supplemental 
A
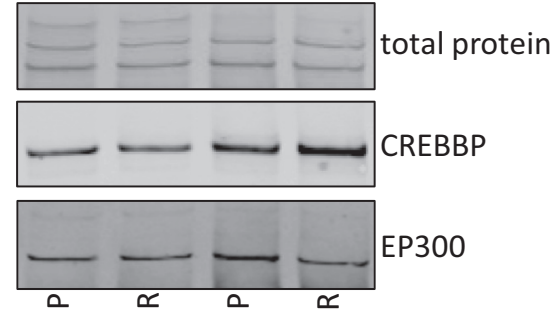

EP300
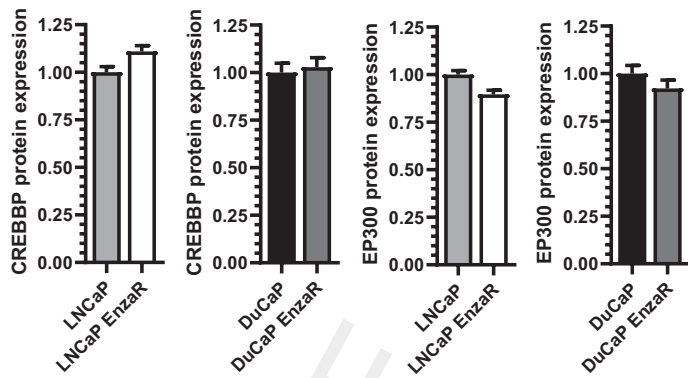

B
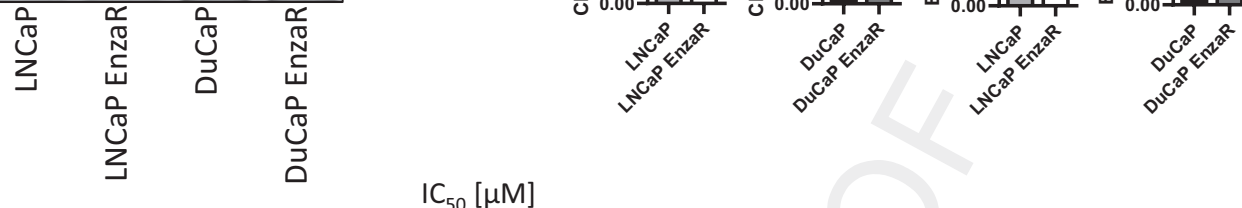

\begin{tabular}{|l|r|r|l|}
\hline & DuCaP & DuCaP EnzaR & Sum-of-Square-F-Test \\
\hline C646 & 17.51 & 8.75 & $* * *$ \\
\hline I-CBP112 & 12.12 & 12.11 & \\
\hline
\end{tabular}

\begin{tabular}{|l|r|r|l|}
\hline & LNCaP & LNCaP EnzaR & Sum-of-Square-F-Test \\
\hline C646 & 4.916 & 3.67 & $*$ \\
\hline I-CBP112 & 24.59 & 13.85 & $* * *$ \\
\hline
\end{tabular}
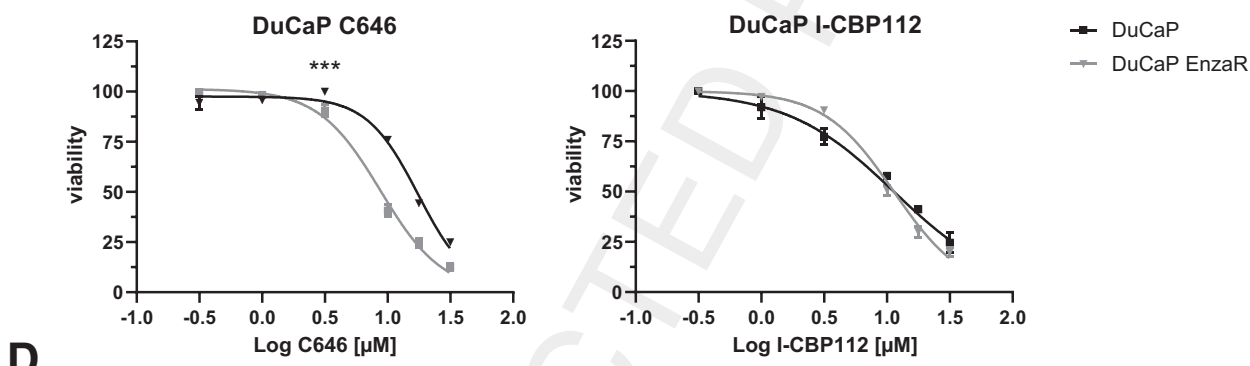

D
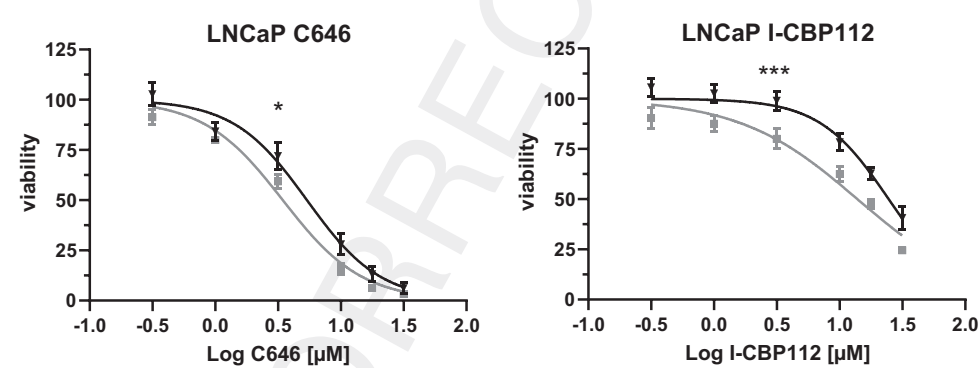

E
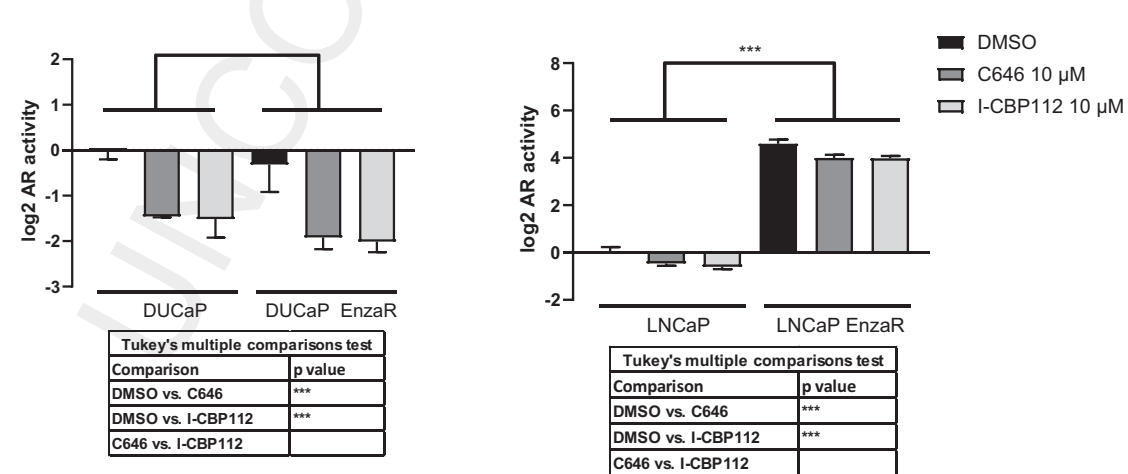

Figure 1 EP300/CREBBP inhibitors in prostate cancer models. A: Representative Western blot of DuCaP and LNCaP parental and enzalutamide-resistant cell lysates probed with anti-EP300 and anti-CREBBP antibodies and quantification relative to parental cells $(n=3)$. B: Fifty percent inhibitory concentration $\left(\mathrm{IC}_{50}\right)$ of EP300/CREBBP inhibitors C646 and I-CBP112. Corresponding dose-response curve for DuCaP (C) and LNCaP (D) parental and enzalutamide-resistant cell lines 72 hours after treatment with the indicated inhibitors based on a viability assay $(n=3)$. E: Dual luciferase reporter assay for parental and resistant DuCaP and LNCaP cell lines treated with $10 \mu \mathrm{M}$ of the respective inhibitor over 24 hours $(n=4)$. Numerical data were analyzed by using two-way analysis of variance. Bars indicate SEM. $P$ values were calculated by using extra-sum-of-square-F-test for $\mathrm{IC}_{50}{ }^{*} P<0.05,{ }^{* *} P<0.001$. AR, androgen receptor; DMS0, dimethyl sulfoxide. 
A

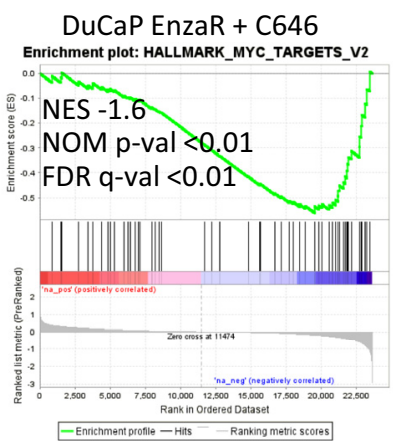

C

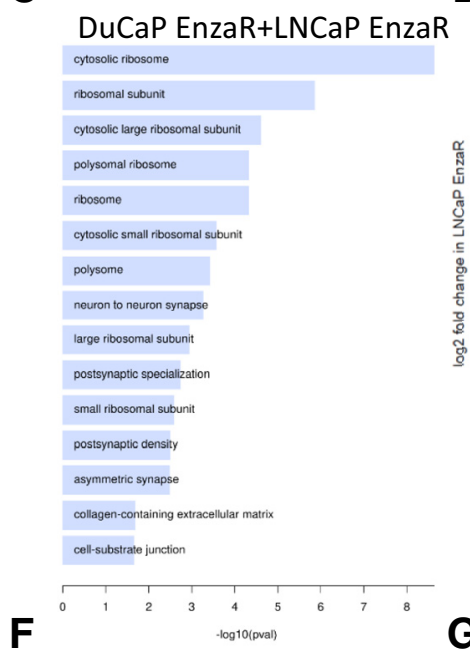

DuCaP EnzaR vs

Parental+Enzalutamide

Enrichment plot: HALLMARK_MYC_TARGETS_V2 ${ }_{\substack{0.45 \\ 0.5}}^{0.03}$ NES 1.37

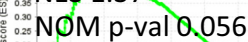

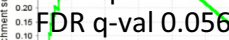

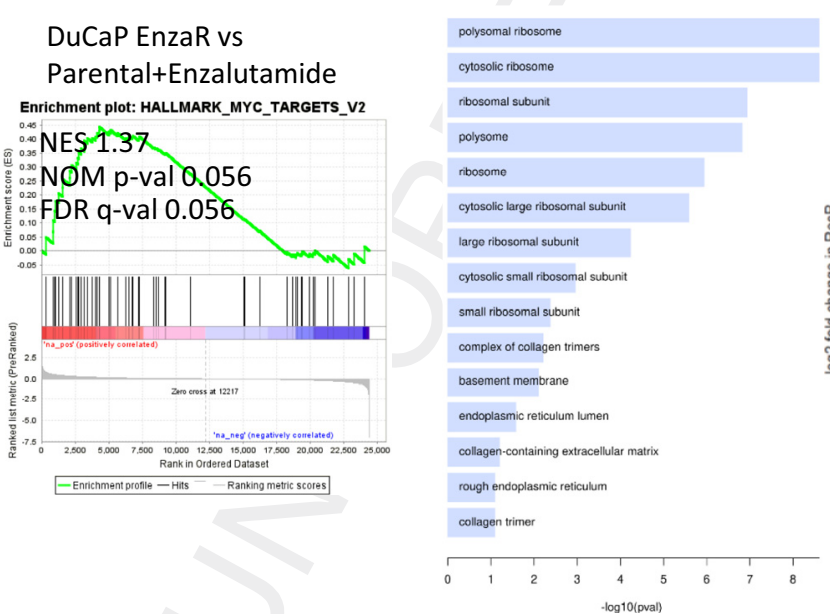

DuCaP EnzaR + I-CBP112

Enrichment plot: HALLMARK_MYC_TARGETS_V2.grp

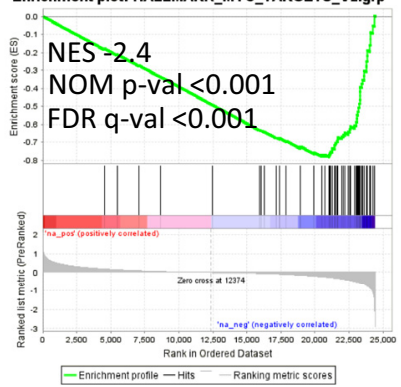

D

G

$\operatorname{Res} A+\operatorname{Res} B$
B
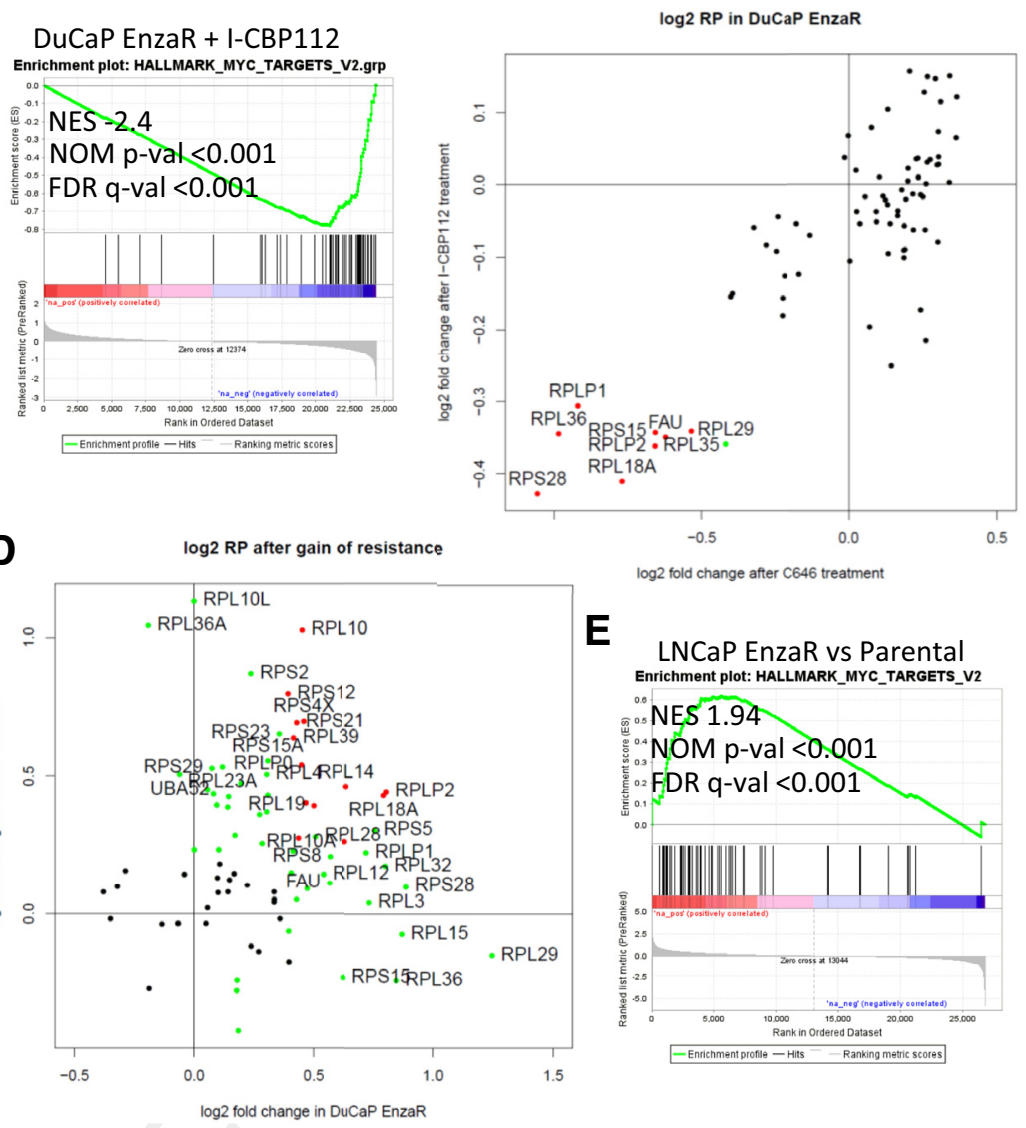

E

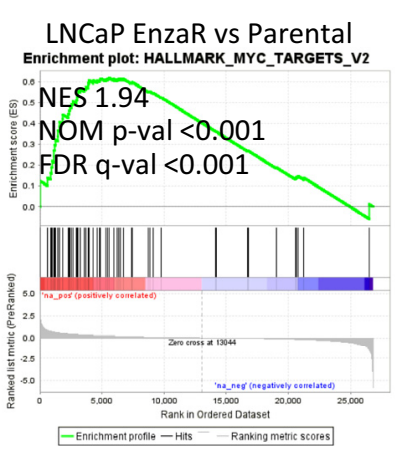

H

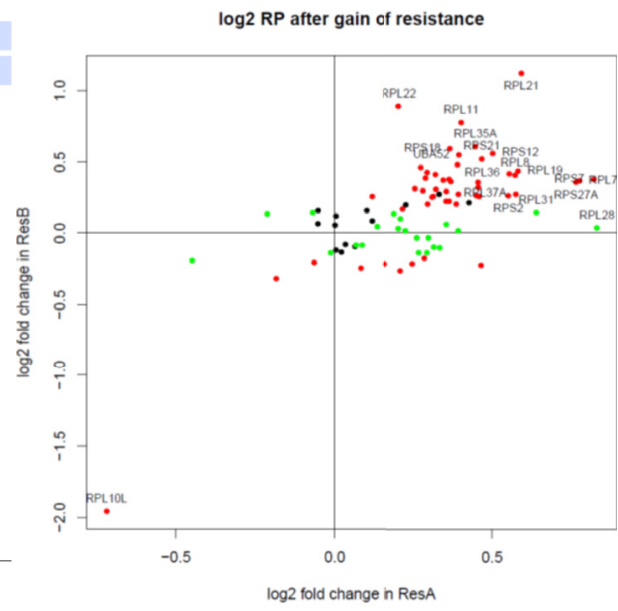

683

684

685

686

687

688

689

690

691

692

693

694

695

696

697

698

699

700

701

702

703

704

705

706

707

708

709

710

711

712

713

714

715

716

717

718

719

720

721

722

723

724

725

726

727

728

729

730

731

732

733

734

735

736

737

738

739

740

741

742

743

744 

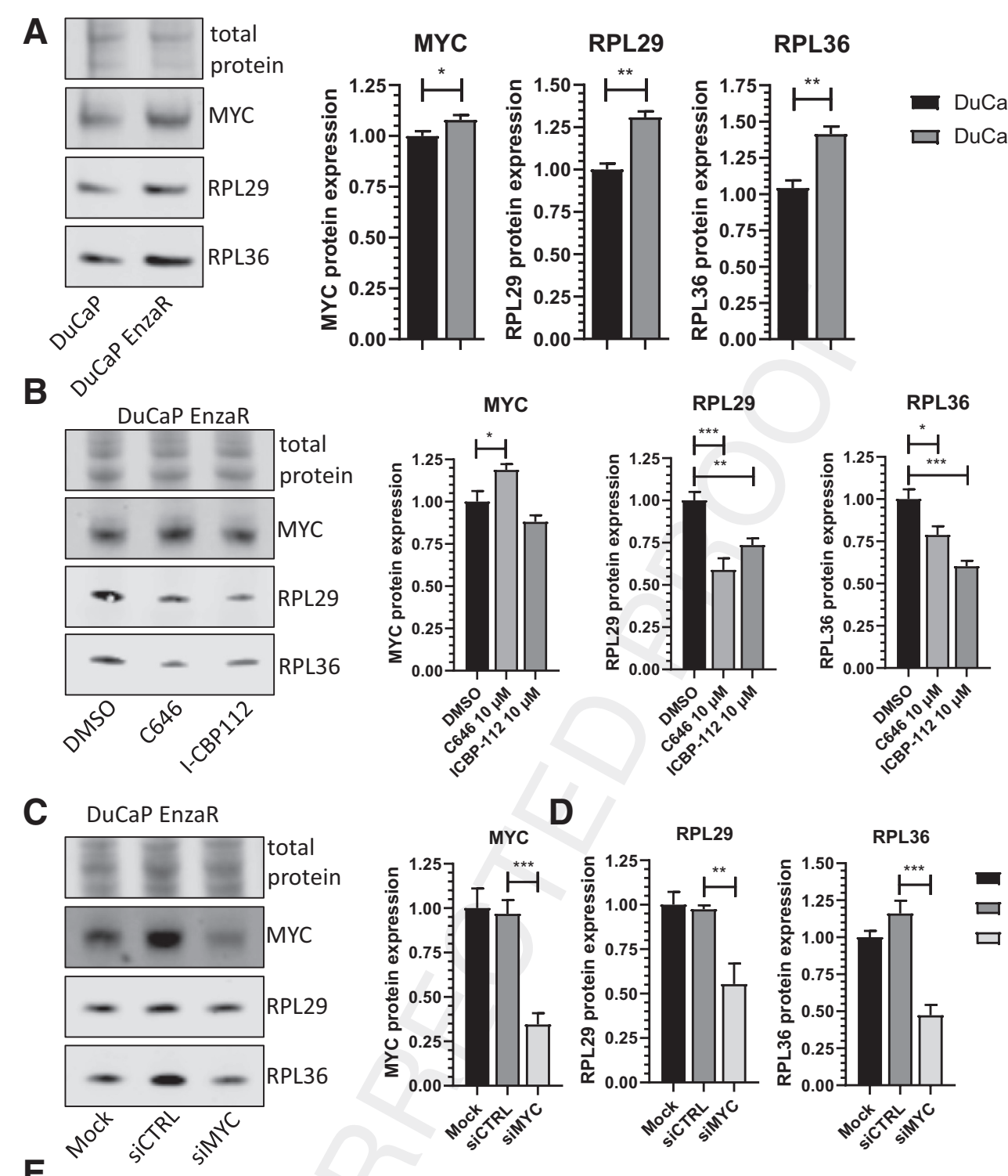

E
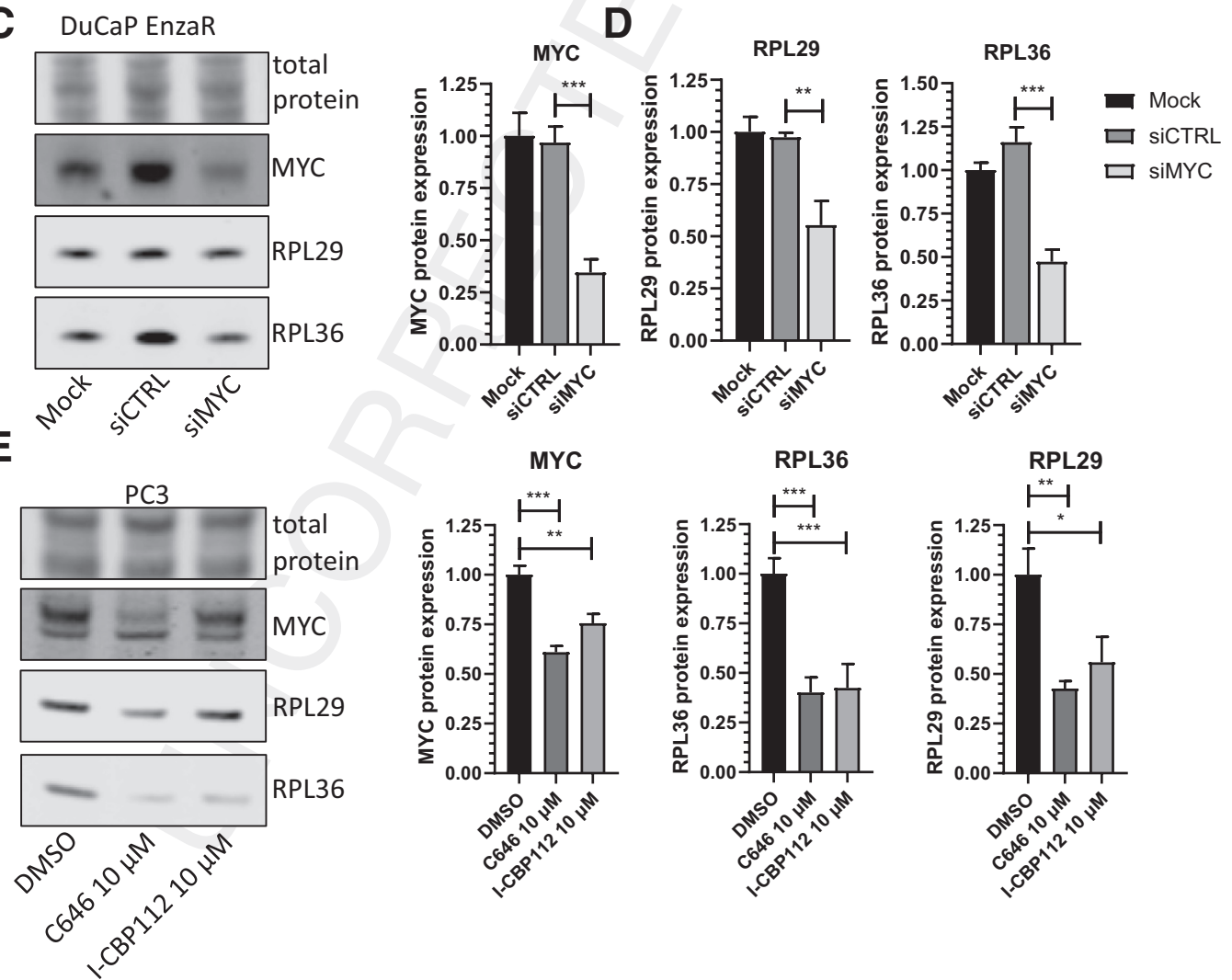

Figure 3 Validation of MYC, RPL29, and RPL36 expression. A: Representative Western blots and quantification of parental and enzalutamide-resistant DuCaP cells probed for MYC, RPL29, and RPL36 $(n=6)$. B: Representative Western blots and quantification for DuCaP EnzaR cells treated with $10 \mu$ M C646, $10 \mu$ M ICBP112, or dimethyl sulfoxide (DMS0) for 48 hours, probed for MYC, RPL29, and RPL36 $(n=6)$. C: Pooled siRNAs either targeting MYC (siMYC) or nontargeting control (siCTRL) were used to establish and validate MYC knockdown together with mock treatment (Mock). Knockdown efficiency was validated by Western blot with an antibody for MYC. D: The blots were also probed with antibodies specific for RPL36 and RPL29. For quantification, MYC, RPL36, and RPL29 were normalized to mock-treated cells $(n=3)$. E: PC3 cells were treated for 48 hours with $10 \mu \mathrm{M}$ C646 or $10 \mu \mathrm{M} \mathrm{I-CBP112}(n=3)$. Blots were probed with MYC, RPL36, and RPL29 specific antibodies. Numerical data were analyzed via one-way analysis of variance. ${ }^{*} P<0.05,{ }^{* *} P<0.01,{ }^{* *} P<0.001$. 
Figure S1C). Of note, the EP300/CREBBP inhibitors led to the same relative AR activity reduction in enzalutamidesensitive and enzalutamide-resistant cell lines. However, the remaining AR activity after inhibitor treatment was still more than 10 times higher in LNCaP EnzaR cells compared with untreated parental cells, which suggests that other pathways are responsible for the observed growth inhibition.

\section{RNA-Sequencing Reveals Regulation of Ribosomal Proteins in Enzalutamide-resistant Cells}

To identify the down-stream effects of the inhibitors, RNAsequencing was performed in DuCaP EnzaR cells treated with $10 \mu \mathrm{M}$ of C646 or I-CBP112 for 24 hours. GSEA pathway analysis revealed a significant reduction of the MYC activity signature after treatment with either inhibitor [F2] (Figure 2A, Supplemental Tables S1-S4). Moreover, Gene Ontology analysis of genes commonly down-regulated in both cell lines (Supplemental Tables S5-S7) revealed structural constituents of the ribosome as an overrepresented term. Further analysis revealed that a subset of genes encoding ribosomal proteins was down-regulated after treatment with either inhibitor (Figure 2B). This finding is in line with previous publications that have shown MYCdependent regulation of ribosomal proteins. ${ }^{34}$

To examine why EP300/CREBBP inhibitors affect enzalutamide-resistant $\mathrm{DuCaP}$ and $\mathrm{LNCaP}$ cells more than enzalutamide-sensitive parental cells, they were also analyzed by RNA-sequencing. Consistent with the above findings, Gene Ontology analysis of genes commonly upregulated in DuCaP EnzaR and LNCaP EnzaR cells compared with untreated parental cell lines revealed an overrepresentation of genes related to the ribosome and collagen-containing extracellular matrix (Figure 2C, Supplemental Tables S8-S10). A more detailed analysis revealed that the majority of genes encoding for ribosomal proteins were up-regulated in at least one cell line, although there was only a partial overlap between the two cell lines (Figure 2D). Consistently, GSEA pathway analysis showed that the MYC activity signature was strongly enriched in LNCaP EnzaR cells (Figure 2E, Supplemental Tables S11 and S12) and moderately activated in DuCaP EnzaR cells, as shown by comparison with enzalutamide-treated parental DuCaP cells (Figure 2F).

To further confirm our results, we analyzed a second publicly available RNA-sequencing data set with two longterm antiandrogen- treated cell lines (ResA and ResB) that are highly resistant to enzalutamide. ${ }^{35}$ Although these cells are also derived from LNCaP, they have low basal AR activity (AR indifferent) in contrast to LNCaP EnzaR cells (Figure 1E). Importantly, we also observed an overrepresentation of up-regulated genes encoding ribosomal genes in ResA and ResB cells compared with normal LNCaP cells (Figure 2, G and $\mathrm{H}$ ).
Ribosomal Proteins Are Up-Regulated in Enzalutamideresistant Cells and Downregulated after EP300/CREBBP Inhibitor Treatment on the Protein Level

We next measured the protein expression of MYC and two of the most strongly regulated ribosomal proteins in DuCaP cells after gain of resistance or treatment with inhibitors, $R P L 36$ and RPL29. In line with our RNA-sequencing data, we observed an increased protein expression of RPL36 and RPL29 in DuCaP EnzaR cells (Figure 3A), which could be reversed by treatment with the EP300/CREBBP inhibitors (Figure 3B). Of note, the inhibitors did not reduce MYC protein expression, suggesting a more complex influence on MYC activity.

To verify if MYC knock-down is sufficient to regulate the expression of ribosomal proteins, a knock-down by siRNA was performed (Figure 3C). Consequently, we found that MYC knock-down is sufficient to down-regulate protein expression of RPL36 and RPL29 (Figure 3D). AR-negative PC 3 cells were used to determine whether EP300/CREBBP inhibitors can reduce ribosomal protein expression also independently of AR. Indeed, both C646 and I-CBP112 were able to reduce RPL36 and RPL29 expression (Figure 3E). MYC was also down-regulated in PC3 cells upon inhibitor treatment.

\section{Ribosomal Proteins Expression in Patients' Samples}

To confirm the clinical relevance of our findings in patients with PCa, we investigated MYC activity and the mRNA expression of ribosomal proteins in primary and metastatic castration-resistant $\mathrm{PCa}$ samples from the Stand Up To Cancer data set. In addition to cell lines, mRNA of ribosomal proteins and MYC were also analyzed in patient data. Indeed, we observed a statistically significant correlation between MYC gene set activity and the mRNA expression of ribosomal proteins (Figure 4A). However, MYC expression is not the only factor that correlated with overexpression of ribosomal proteins. Copy number aberrations are common for ribosomal proteins (Figure 4B). Interestingly, high-level amplifications of $R P L 7, R P L 30, R P L 8$, and RPS20 are common in up to $27 \%$ of patients. All three amplifications co-occur frequently with amplifications of $M Y C$, as shown by clustering (Figure 4B). This is not surprising because they are all located in proximity on chromosome 8. Amplifications of parts of chromosome 8 are common. ${ }^{36}$ However, ribosomal proteins not located on chromosome 8, including RPS27 and RPS24, were also highly amplified in $10 \%$ of patients.

\section{Inhibition of Ribosomal Proteins Affects Cellular Viability and Migration in PCa}

Although C646 and I-CBP112 are useful tools for research, they are not well suited for clinical therapy due to their low half-life and selectivity. ${ }^{37,38}$ To overcome this problem, we ${ }^{114}$ 
A

SU2C dataset - geneset activity score

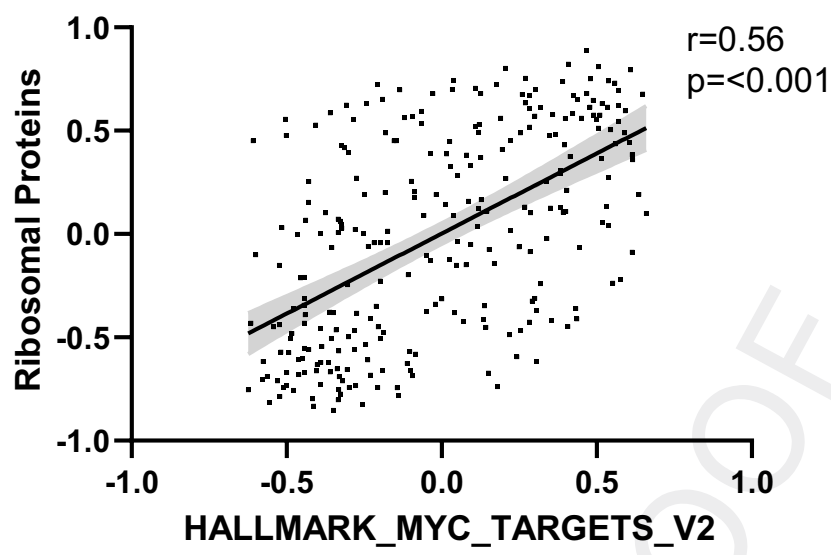

B

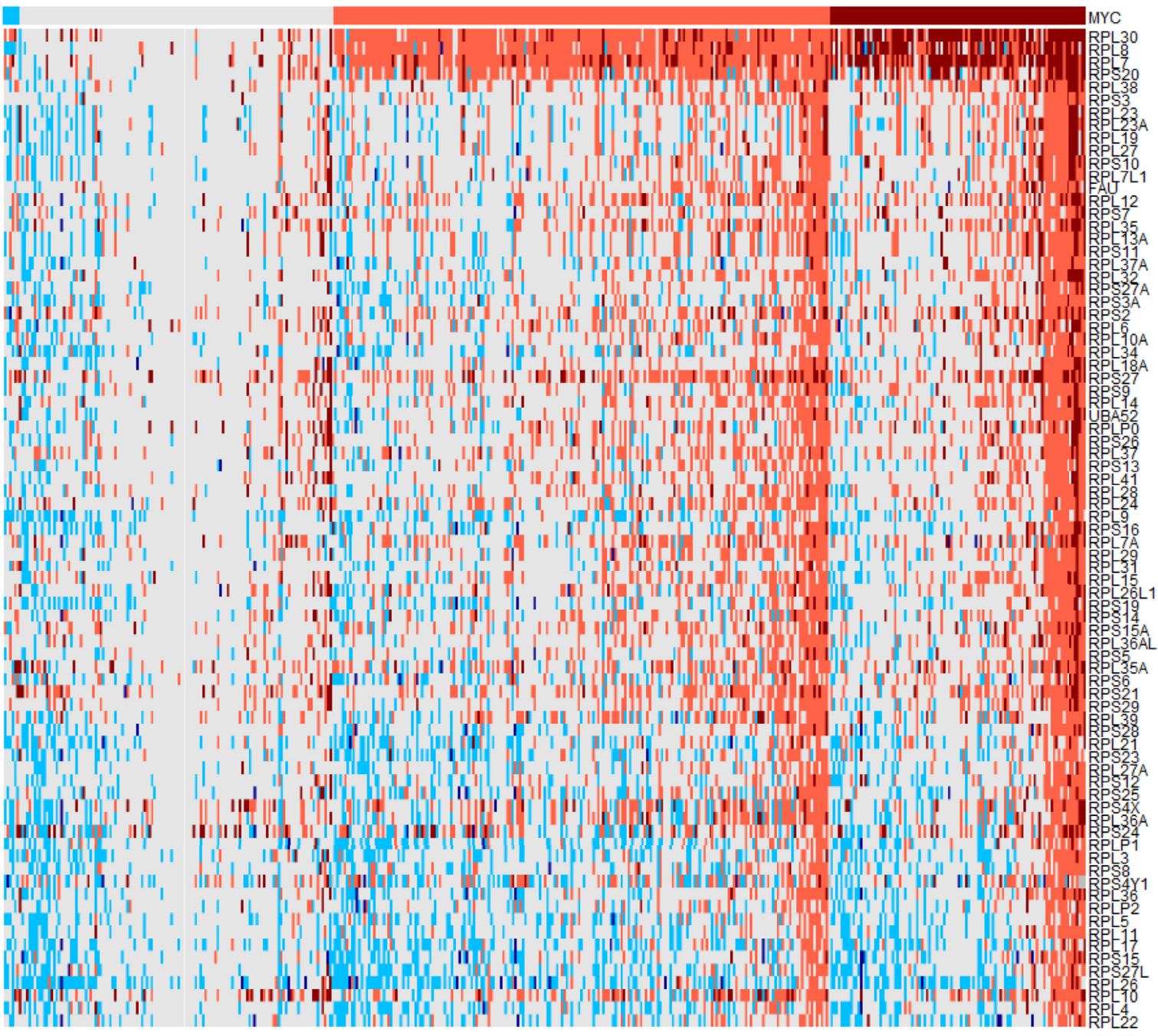

Deep Deletion

\section{Shallow Deletion}

Normal
Gain

\section{Amplification}

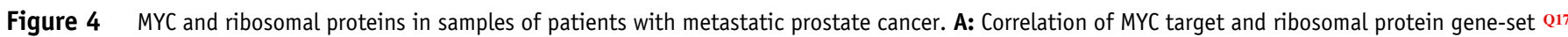
variation analysis scores from the publicly available Stand Up To Cancer data sets for metastatic prostate cancer. Graph is shown with linear regression (black line) and $95 \%$ confidence intervals (gray shaded area). The $r$ score and $P$ value were calculated by using Pearson correlation. B: Correlation of copy number aberrations in genes for ribosomal proteins and MYC. Copy number aberrations are separated as high-level amplification (2), low-level gain (1), diploid (0), shallow loss $(-1)$, and deep loss $(-2)$.

1055

1056

1057

1058

1059

1060

1061

1062

1063

1064

1065

1066

1067

1068

1069

1070

1071

1072

1073

1074

1075

1076

1077

1078

1079

1080

1081

1082

1083

1084

1085

1086

1087

1088

1089

1090

1091

1092

1093

1094

1095

1096

1097

1098

1099

1100

1101

1102

1103

1104

1105

1106

1107

1108

1109

1110

1111

1112

1113

1114

1115

1116 
Furlan et al
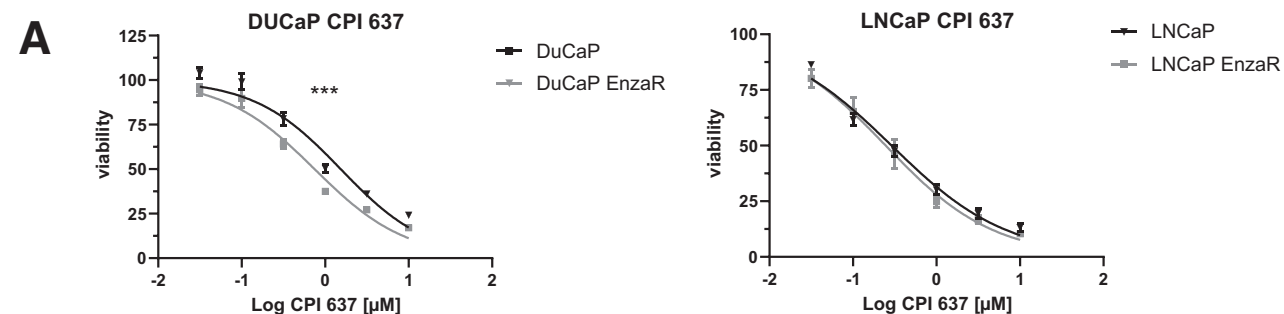

B
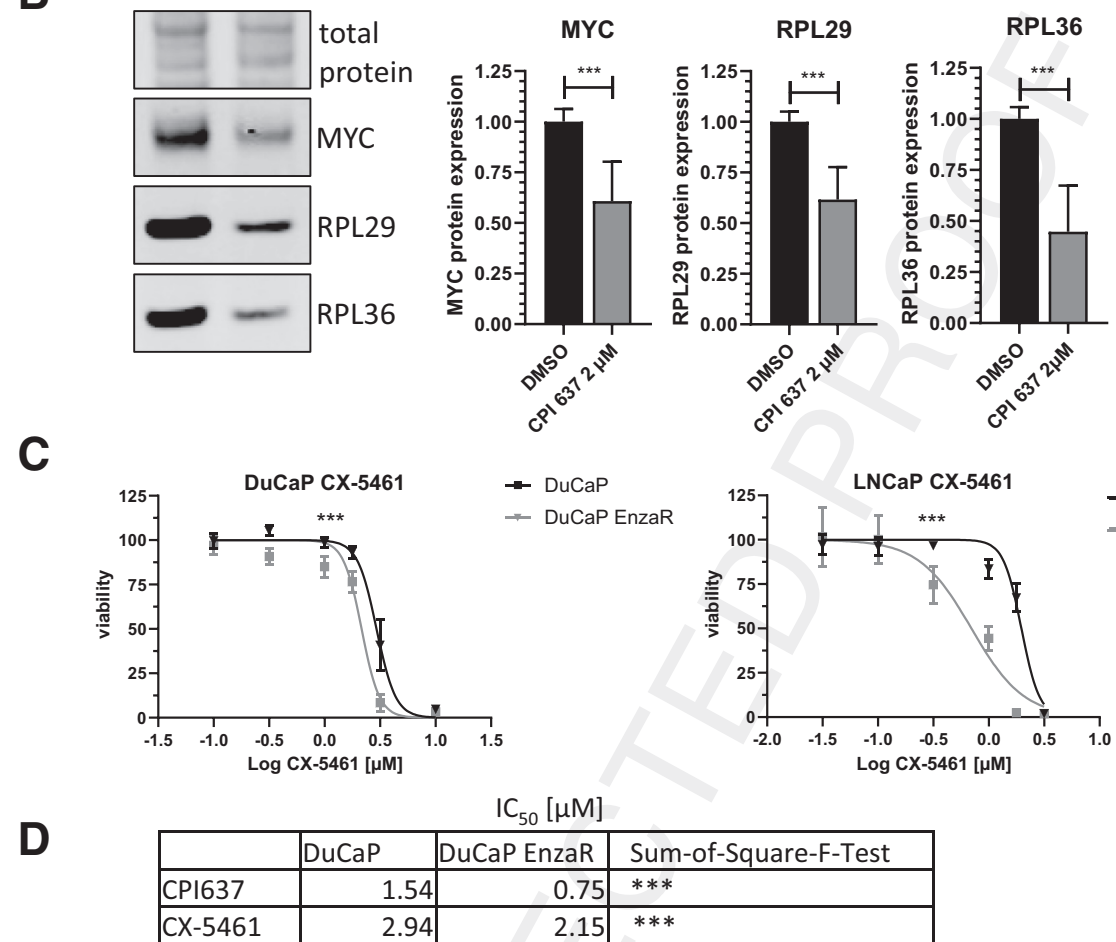

\begin{tabular}{|c|c|c|c|}
\hline & DuCaP & DuCaP EnzaR & Sum-of-Square-F-Test \\
\hline CPI637 & 1.54 & 0.75 & $* * *$ \\
\hline CX-5461 & 2.94 & 2.15 & $* * *$ \\
\hline & LNCaP & LNCaP EnzaR & Sum-of-Square-F-Test \\
\hline CPI637 & 0.29 & 0.26 & \\
\hline CX-5461 & 1.97 & 0.69 & $* * *$ \\
\hline
\end{tabular}

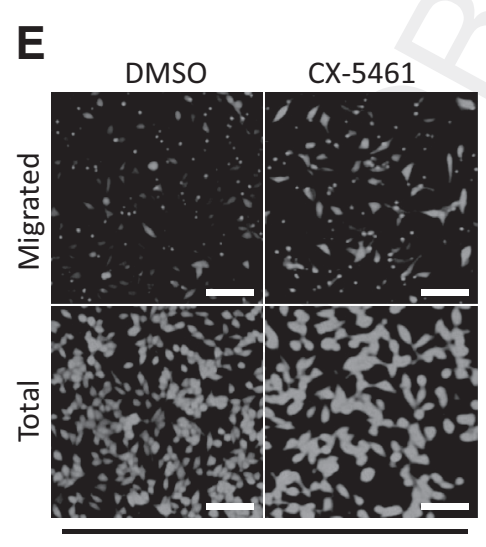

LNCaP

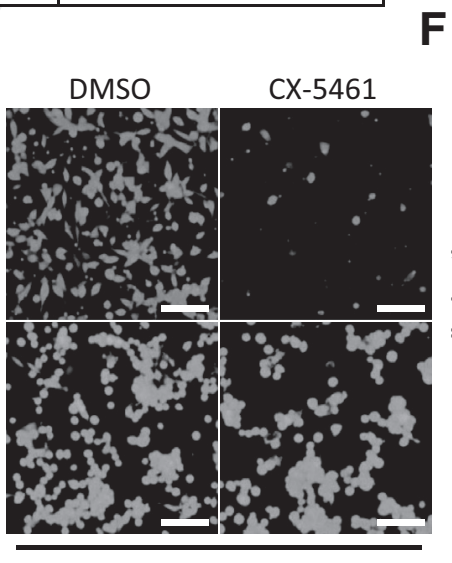

LNCaP EnzaR

\section{F}

* LNCaP

- LNCaP EnzaR

Figure 5 Next-generation bromodomain inhibitor and ribosomal neogenesis inhibitor in prostate cancer cell lines. A: Dose-response curve for parental and resistant DuCaP and LNCaP cells after 72 hours of CPI637 treatment. B: Representative Western blots for DuCaP EnzaR cells treated with $2 \mu$ M CPI637 for 48 hours, probed for MYC, RPL29, and RPL36 $(n=3)$. C: Dose-response curve for parental and resistant DuCaP and LNCaP cells after 72 hours of CX-5461 treatment. D: Fifty percent inhibitory concentration (IC $C_{50}$ ) of CPI637 and CX-5461 for DuCaP and LNCaP parental and enzalutamide-resistant cell lines 72 hours after treatment based on a viability assay $(n=3)$. $P$ value was calculated using extra-sum-of-square-F-test for $\mathrm{IC}_{50}$. E: Boyden chamber assay with parental and LNCaP EnzaR cells, with or without $2 \mu \mathrm{M}$ CX-5461 for 72 hours. Cells were stained with Calcein AM. Upper row shows migrated cells, and lower row shows total cells. (F) Graph shows relative fluorescence intensity to untreated cells, normalized to wells with same treatment but without insert. Numerical data were analyzed via one-way analysis of variance. Bars indicate SEM $(n=3)(\mathbf{A}$ and $\mathbf{C}) .{ }^{* * * P}<0.001$. Scale bars: $100 \mu \mathrm{m}(\mathbf{E})$. DMSO, dimethyl sulfoxide. 
tested CPI637, a newer generation BD inhibitor ${ }^{39}$ (Figure 5, [F5] A and B). CPI637 reached a nanomolar 50\% inhibitory concentration in both enzalutamide-resistant cell lines tested, and a higher efficiency could be observed in DuCaP EnzaR cells compared with enzalutamide-sensitive parental cells (Figure 5C).

Our results showed that ribosomal proteins were upregulated not only in enzalutamide-resistant cells but also in patients' samples. To investigate the potential utility of targeting ribosomal proteins, we used an inhibitor of RNA polymerase I-directed transcription (CX-5461), which is responsible for transcription of rRNA and thereby inhibits ribosome formation. Indeed, enzalutamide-resistant cells were significantly more susceptible to CX-5461 than their enzalutamide-sensitive parental counterparts (Figure 5D).

Other studies have shown that expression of ribosomal proteins correlates with increased migration and invasion potential. ${ }^{40,41}$ To test whether and to what extent ribosomal proteins regulate migration in our cell models, a migration assay was performed with parental LNCaP and LNCaP EnzaR cell lines. DuCaP cells are not suited for such assays due to their growth pattern. CX-5461 treatment of enzalutamide-resistant LNCaP cells but not parental cells resulted in significantly lower migration (Figure 5, E and F).

\section{Discussion}

It is well known that EP300/CREBBP function as oncogenes in $\mathrm{PCa}$ via potentiation of ligand-independent $\mathrm{AR}$ activation, regulation of $\mathrm{AR}$ target gene expression in androgen-insensitive cells, and activation of cellular processes such as migration and invasion. ${ }^{4,8,42}$ Recently, we have identified EP300 also as a target in chemotherapyresistant PCa cells. ${ }^{13}$ Furthermore, EP300 and CREBBP are up-regulated during androgen ablation. ${ }^{12}$ As with EP300, CREBBP is involved in regulating the antagonist/ agonist balance of antiandrogens. ${ }^{10}$ Thus, CREBBP and EP300 may have distinct functions in prostate carcinogenesis, depending on tumor stage and cell model used.

Several different mechanisms of enzalutamide resistance have been discovered thus far, including mutations and amplification of the $A R,{ }^{43}$ enhanced activity of co-activators such as GREB $1,{ }^{44}$ or increased activity of the glucocorticoid receptor. $^{21,45,46}$ Overexpression of $\mathrm{AR}$, the AR variant 7, and glucocorticoid receptor have been identified previously in the cell models used in this study. ${ }^{21,22}$ In addition, we show here that MYC signaling is elevated in enzalutamideresistant cell models. The highest expression of wild-type and variant AR in cellular models developed by our laboratory was seen in DuCaP cells, which were also used in the current study.

Interestingly, lower concentrations of EP300/CREBBP inhibitors were sufficient to decrease viability in several models of enzalutamide-resistant cells compared with their nonresistant parental counterparts. However, as shown in luciferase assays, this decreased viability in response to both inhibitors was not caused by decreased AR activity. We therefore performed RNA-sequencing to identify potential molecular basis for reduced viability in cells treated with BD/HAT inhibitors. Our RNA-sequencing experiments revealed that enzalutamide resistance was associated with some changes in both cell lines. Although modulation of AR signaling differed between DuCaP EnzaR and LNCaP EnzaR cells, overexpression of ribosomal proteins and activation of MYC signaling was a common factor, confirmed by bioinformatics analysis. Although we observed an up-regulation of many ribosomal proteins in DuCaP EnzaR and LNCaP EnzaR cells, approximately onethird of ribosomal proteins showed no altered expression. This finding suggests that the overexpressed ribosomal proteins may not be involved in ribosome formation per se, which would require a homogeneous distribution of ribosomal proteins. Notably, a shift in the transcriptome has been reported for heterogeneous ribosomes. ${ }^{47}$

For validation of these particular RNA-sequencing findings, we quantified two proteins, RPL36 and RPL29, that were overexpressed in enzalutamide-resistant DuCaP cells and down-regulated upon BD/HAT inhibitor treatment. A potential role of other ribosomal proteins in PCa pathogenesis has previously been reported, including RPL19, whose knockdown in the AR-negative cell line PC3 reduced invasive potential and tumorigenicity. ${ }^{40}$ Signaling pathway analysis of RPL19-depleted cells identified changes in transcription factor networks and cellular adhesion genes. Besides RPL19, the ribosomal proteins 21 and 24 have been proposed as possible PCa biomarkers. ${ }^{48}$ Taken together, previous results and those reported here open the possibility to investigate multiple roles of ribosomal proteins in $\mathrm{PCa}$ and their contribution to therapy resistance.

Up-regulation of ribosomal proteins is not a peculiarity of our cell models but was also observed in those reported by Handle et al. ${ }^{35}$ Interestingly, in that study, AR activity was not required for growth of enzalutamide-resistant cells. In the current study, we show that our enzalutamide-resistant cell lines are also more susceptible toward an inhibitor of ribosomal biogenesis, which not only reduced viability but also cell migration. These results indirectly confirm those of Bee et al, ${ }^{40}$ who emphasized the importance of ribosomal proteins for the invasive phenotype. Our results may be particularly interesting in view of those recently published by Ebright at al, ${ }^{41}$ wherein RPL15 overexpression promoted metastatic breast cancer growth. The authors also reported that circulating tumor cells from patients with breast cancer displayed ribosome and protein synthesis signatures. Taken together, data obtained in prostate and breast cancer studies indicate that ribosomal proteins may have a key role in the progression of endocrine-related malignancies. It remains to be investigated whether specific subgroups of ribosomal proteins have redundant functions in cancer. The responsiveness of enzalutamide-resistant cells to CX-5461 in viability and 
migration studies was similar to that observed with EP300/ CREBBP inhibitors.

To analyze EP300/CREBBP action in enzalutamideresistant cells in detail, we inhibited MYC function in the current study via siRNA-mediated knockdown. It was shown that MYC knockdown led to the down-regulation of several ribosomal proteins. In addition, analysis of patient data confirmed that up-regulation of ribosomal proteins correlates with MYC activity. These results are consistent with several previous reports indicating ribosomal protein regulation by MYC and the established role of MYC as a regulator of ribosome biogenesis, function, and protein synthesis. $^{49}$ Involvement of MYC overexpression as a mechanism for resistance to enzalutamide has been previously reported, in part in cells that display reduced AR expression when enzalutamide resistance occurs. ${ }^{35,50} \mathrm{In}$ teractions between MYC and the AR signaling pathway are bidirectional. Treatment with EP300/CREBBP inhibitors not only reduced AR but also MYC signaling. ${ }^{51}$

Although our study focused on inhibition of EP300/ CREBBP in association with MYC/ribosomal protein regulation, other possible actions of these inhibitors should be mentioned. Recently, Fan et $\mathrm{al}^{52}$ reported that enzalutamide-resistant $\mathrm{C} 4-2$ cells are more sensitive to either BET or EP300/CREBBP inhibitors than parental cells. They attributed the effect to their destabilization of the histone demethylase JMJD1A, which is acetylated by EP300. Importantly, our results, which were obtained using different models compared with those of Fan et al, are in concordance regarding the use of those inhibitors in enzalutamide-resistant $\mathrm{PCa}$.

Previous studies using EP300/CREBBP inhibitors in a PCa background suggested that suppression of AR signaling is responsible for the observed inhibition of proliferation. Here, we show that inhibition of MYC signaling and subsequent down-regulation of ribosomal proteins are also involved. This correlates with our previous results, which showed that the EP300/CREBBP HAT inhibitor C646 is also effective in the AR-negative cell line PC $3 .{ }^{53}$ In clinical trials for acute myeloid leukemia, EP300 and CREBBP inhibitors were shown to have limited side effects and cytotoxic effects. For the treatment of $\mathrm{PCa}$ with $\mathrm{BD}$ inhibitors, phase $2 \mathrm{a}$ clinical trials have also been undertaken and show promising results. ${ }^{54}$ In recent years, generations of EP300/CREBBP inhibitors that exhibit higher specificity and potency have been developed, suggesting that results from previous clinical trials could be further improved.

In conclusion, our results reveal that targeting EP300/ CREBBP in advanced $\mathrm{PCa}$ is not limited to AR signaling but also involves the MYC/ribosomal protein axis. Findings reported herein open possibilities to elucidate the role of ribosomal proteins in PCa. Therefore, clinical targeting of ribosomal proteins either through newer generation of EP300/CREBBP inhibitors or directly targeting ribosomes may be a promising strategy to treat advanced $\mathrm{PCa}$ in the future.

\section{Acknowledgments}

We thank Lisa Schnaller and Alexandra Kaindl-Lindinger for technical support. We also thank all members of Experimental Urology, Medical University of Innsbruck, for helpful discussions.

\section{Supplemental Data}

Supplemental material for this article can be found at http://doi.org/10.1016/j.ajpath.2021.02.017.

\section{References}

1. Barsouk A, Padala SA, Vakiti A, Mohammed A, Saginala K, Thandra KC, Rawla P, Barsouk A: Epidemiology, staging and management of prostate cancer. Med Sci (Basel) 2020, 8:28

2. Tran C, Ouk S, Clegg NJ, Chen Y, Watson PA, Arora V, Wongvipat J, Smith-Jones PM, Yoo D, Kwon A, Wasielewska T, Welsbie D, Degui Chen C, Higano CS, Beer TM, Hung DT, Scher HI, Jung ME, Sawyers CL: Development of a secondgeneration antiandrogen for treatment of advanced prostate cancer. Science 2009, 324:787-790

3. Prekovic S, van der Broeck T, Linder S, van Royen ME, Houtsmuller AB, Handle F, Joniau S, Zwart W, Claessens F: Molecular underpinnings of enzalutamide resistance. Endocr Relat Cancer 2018, 25:R545-R557

4. Debes JD, Schmidt LJ, Huang H, Tindall DJ: p300 Mediates androgen-independent transactivation of the androgen receptor by interleukin 6. Cancer Res 2002, 62:5632-5636

5. Dancy BM, Cole PA: Protein lysine acetylation by p300/CBP. Chem Rev 2015, 115:2419-2452

6. Chen J, Ghazawi FM, Li Q: Interplay of bromodomain and histone acetylation in the regulation of p300-dependent genes. Epigenetics 2010, 5:509-515

7. Weinert BT, Narita T, Satpathy S, Srinivasan B, Hansen BK, Schölz C, Hamilton WB, Zucconi BE, Wang WW, Liu WR, Brickman JM, Kesicki EA, Lai A, Bromberg KD, Cole PA, Choudhary C: Time-resolved analysis reveals rapid dynamics and broad scope of the CBP/p300 acetylome. Cell 2018, 174:231-244. e12

8. Fu M, Wang C, Reutens AT, Wang J, Angeletti RH, SiconolfiBaez L, Ogryzko V, Avantaggiati ML, Pestell RG: p300 and p300/cAMP-response element-binding protein-associated factor acetylate the androgen receptor at sites governing hormone-dependent transactivation. J Biol Chem 2000, 275:20853-20860

9. Vervoorts J, Lüscher-Firzlaff JM, Rottmann S, Lilischkis R, Walsemann G, Dohmann K, Austen M, Lüscher B: Stimulation of cMYC transcriptional activity and acetylation by recruitment of the cofactor CBP. EMBO Rep 2003, 4:484-490

10. Comuzzi B, Nemes C, Schmidt S, Jasarevic Z, Lodde M, Pycha A, Bartsch G, Offner F, Culig Z, Hobisch A: The androgen receptor coactivator CBP is up-regulated following androgen withdrawal and is highly expressed in advanced prostate cancer. J Pathol 2004, 204: $159-166$

11. Ianculescu I, Wu DY, Siegmund KD, Stallcup MR: Selective roles for cAMP response element-binding protein binding protein and p300 protein as coregulators for androgen-regulated gene expression in advanced prostate cancer cells. J Biol Chem 2012, 287:4000-4013

12. Heemers HV, Sebo TJ, Debes JD, Regan KM, Raclaw KA, Murphy LM, Hobisch A, Culig Z, Tindall DJ: Androgen deprivation increases p300 expression in prostate cancer cells. Cancer Res 2007, $67: 3422-3430$ 
13. Gruber M, Ferrone L, Puhr M, Santer FR, Furlan T, Eder IE Sampson N, Schäfer G, Handle F, Culig Z: p300 is upregulated by docetaxel and is a target in chemoresistant prostate cancer. Endocr Relat Cancer 2020, 27:187-198

14. Yan G, Eller MS, Elm C, Larocca CA, Ryu B, Panova IP, Dancy BM, Bowers EM, Meyers D, Lareau L, Cole PA, Taverna SD, Alani RM: Selective inhibition of p300 HAT blocks cell cycle progression, induces cellular senescence, and inhibits the DNA damage response in melanoma cells. J Invest Dermatol 2013, $133: 2444-2452$

15. Liu Y, Yang EJ, Shi C, Mou PK, Zhang B, Wu C, Lyu L, Shim JS: Histone acetyltransferase (HAT) P300/CBP inhibitors induce synthetic lethality in PTEN-deficient colorectal cancer cells through destabilizing AKT. Int J Biol Sci 2020, 16:1774-1784

16. Giotopoulos G, Chan WI, Horton SJ, Ruau D, Gallipoli P, Fowler A, Crawley C, Papaemmanuil E, Campbell PJ, Göttgens B, Van Deursen JM, Cole PA, Hunthly BJP: The epigenetic regulators CBP and p300 facilitate leukemogenesis and represent therapeutic targets in acute myeloid leukemia. Oncogene 2016, 35:279-289

17. van Bokhoven A, Varella-Garcia M, Korch C, Johannes WU, Smith EE, Miller HL, Nordeen SK, Miller GJ, Lucia MS: Molecular characterization of human prostate carcinoma cell lines. Prostate 2003, 57:205-225

18. Horoszewicz JS, Leong SS, Kawinski E, Karr JP, Rosenthal H, Chu TM, Mirand EA, Murphy GP: LNCaP model of human prostatic carcinoma. Cancer Res 1983, 43:1809-1818

19. Lee YG, Korenchuk S, Lehr J, Whitney S, Vessela R, Pienta KJ: Establishment and characterization of a new human prostatic cancer cell line: DuCaP. In Vivo 2001, 15:157-162

20. Saramäki OR, Harjula AE, Martikainen PM, Vessella RL, Tammela TLJ, Visakorpi T: TMPRSS/ERG fusion identifies a subgroup of prostate cancers with a favorable prognosis. Clin Cancer Res 2008, 14:3395-3400

21. Puhr M, Hoefer J, Eigentler A, Ploner C, Handle F, Schaefer G, Kroon J, Leo A, Heidegger I, Eder I, Culig Z, Van der Pluijm G, Klocker $\mathrm{H}$ : The glucocorticoid receptor is a key player for prostate cancer cell survival and a target for improved antiandrogen therapy. Clin Cancer Res 2018, 24:927-938

22. Hoefer J, Akbor M, Handle F, Ofer P, Puhr M, Parson W, Culig Z, Klocker H, Heidegger I: Critical role of androgen receptor level in prostate cancer cell resistance to new generation antiandrogen enzalutamide. Oncotarget 2016, 7:59781-59794

23. Puhr M, Santer FR, Neuwirt H, Susani M, Nemeth JA, Hobisch A, Kenner L, Culig Z: Down-regulation of suppressor of cytokine signaling-3 causes prostate cancer cell death through activation of the extrinsic and intrinsic apoptosis pathways. Cancer Res 2009, 69: $7375-7384$

24. Afgan E, Baker D, Batut B, van den Beek M, Bouvier D, Cech M, Chilton J, Clements D, Coraor N, Grüning BA, Guerler A, HillmanJackson J, Hiltermann S, Jalili V, Rasche H, Soranzo N, Goecks J, Taylor J, Nekrutenko A, Blankenberg D: The Galaxy platform for accessible, reproducible and collaborative biomedical analyses: 2018 update. Nucleic Acids Res 2018, 46:W537-W544

25. Kim D, Langmead B, Salzberg SL: HISAT: a fast spliced aligner with low memory requirements. Nat Meth 2015, 12:357-360

26. Anders S, Pyl PT, Huber W: HTSeq — a Python framework to work with high-throughput sequencing data. Bioinformatics 2015, 31 $166-169$

27. Love MI, Huber W, Anders S: Moderated estimation of fold change and dispersion for RNA-seq data with DESeq2. Genome Biol 2014 15:550

28. Ashburner M, Ball CA, Blake JA, Botstein D, Butler H, Cherry JM, Davis AP, Dolinski K, Dwight SS, Eppig JT, Harris MA, Hill DP, Issel-Tarver L, Kasarskis A, Lewis S, Matese JC, Richardson JE, Ringwald M, Rubin GM, Sherlock G: Gene ontology: tool for the unification of biology. The Gene Ontology Consortium. Nat Genet $2000,25: 25-29$
29. Yu G, Wang LG, Han Y, He QY: clusterProfiler: an R package for comparing biological themes among gene clusters. OMICS 2012, 16: 284-287

30. Subramanian A, Tamayo P, Mootha VK, Mukherjee S, Ebert BL, Gillette MA, Paulovich A, Pomeroy SL, Golub TR, Lander ES, Mesirov JP: Gene set enrichment analysis: a knowledge-based approach for interpreting genome-wide expression profiles. Proc Natl Acad Sci U S A 2005, 102:15545-15550

31. Abida W, Cyrta J, Heller G, Prandi D, Amenia J, Coleman I, et al Genomic correlates of clinical outcome in advanced prostate cancer. Proc Natl Acad Sci U S A 2019, 116:11428-11436

32. Bowers EM, Yan G, Mukherjee C, Orry A, Wang L, Holbert MA, Crump NT, Hazzalin CA, Lisczak G, Yuan H, Larocca C, Saldanha SA, Abagyarn R, Sun Y, Meyers DJ, Marmorstein R, Mahadevan LC, Alani RM, Cole PA: Virtual ligand screening of the p300/CBP histone acetyltransferase: identification of a selective small molecule inhibitor. Chem Biol 2010, 17:471-482

33. Picaud S, Fedorov O, Thanasopoulou A, Leonards K, Jones K, Meier J, et al: Generation of a selective small molecule inhibitor of the CBP/p300 bromodomain for leukemia therapy. Cancer Res 2015 , 75:5106-5119

34. Ji H, Wu G, Zhan X, Nolan A, Koh C, De Marzo A, Mai Doan H, Fan J, Cheadle C, Fallahi M, Cleveland JL, Dang CV, Zeller KI Cell-type independent MYC target genes reveal a primordial signature involved in biomass accumulation. PLoS One 2011, 6 e26057

35. Handle F, Prekovic S, Helsen C, Van den Broeck T, Smeets E, Moris L, Eerlings R, El Kharraz S, Urbanucci A, Mills IG, Joniau S, Attard, Claessens F: Drivers of AR indifferent anti-androgen resistance in prostate cancer cells. Sci Rep 2019, 9:13786

36. El Gammal AT, Brüchmann M, Zustin J, Isbarn H, Hellwinkel OJC Köllermann J, Sauter G, Simon R, Wilczak W, Schwarz J, Bokemeyer C, Brümmendorf TH, Izbicki JR, Yekebas E, Fisch M, Huland H, Graefen M, Schlomm T: Chromosome 8p deletions and 8c gains are associated with tumor progression and poor prognosis in prostate cancer. Clin Cancer Res 2010, 16:56-64

37. Cermakova K, Hodges HC: Next-generation drugs and probes for chromatin biology: from targeted protein degradation to phase separation. Molecules 2018, 23:1958

38. Shortt J, Ott CJ, Johnstone RW, Bradner JE: A chemical probe toolbox for dissecting the cancer epigenome. Nat Rev Cancer 2017 $17: 160-168$

39. Taylor AM, Côté A, Hewitt MC, Pastor R, Leblanc Y, Nasveschuk CG, et al: Fragment-based discovery of a selective and cell-active benzodiazepinone CBP/EP300 bromodomain inhibitor (CPI-637). ACS Med Chem Lett 2016, 7:531-536

40. Bee A, Brewer D, Beesley C, Dodson A, Forootan S, Dickinson T, Gerard P, Lane B, Yao S, Cooper CS, Djamgoz MBA, Gosden CM, Ke Y, Foster CS: siRNA knockdown of ribosomal protein gene RPL19 abrogates the aggressive phenotype of human prostate cancer PLoS One 2011, 6:e22672

41. Ebright RY, Lee S, Wittner BS, Niederhoffer KL, Nicholson BT, Bardia A, Truesdell S, Wiley DF, Wesley B, Li S, Mai A, Aceto N, Vincent-Jordan N, Szabolcs A, Chirn B, Kreuzer J, Comaills V, Kalinich M, Haas W, Ting DT, Toner M, Vasudevan S, Haber DA, Maheswaran S, Micalizzi DS: Deregulation of ribosomal protein expression and translation promotes breast cancer metastasis. Science 2020, 367:1468-1473

42. Shin SH, Lee GY, Lee M, Kang J, Shin HW, Chun YS, Park JW: Aberrant expression of CITED2 promotes prostate cancer metastasi by activating the nucleolin-AKT pathway. Nat Comm 2018, 9:4113

43. Blatt EB, Raj GV: Molecular mechanisms of enzalutamide resistance in prostate cancer. Cancer Drug Resist 2019, 2:189-197

44. Lee E, Wongvipat J, Choi D, Wang P, Lee YS, Zheng D, Watson PA, Gopalan A. Sawyers CL: GREB1 amplifies androgen receptor output in human prostate cancer and contributes to antiandrogen resistance. Elife 2019, 8:e41913 
45. Shah N, Wang P, Wongvipat J, Karthaus WR, Abida W, Armenia J, Rockowitz S, Drier Y, Bernstein BE, Long HW, Mreedman ML, Arora VK, Zheng D, Sawyers CL: Regulation of the glucocorticoid receptor via a BET-dependent enhancer drives antiandrogen resistance in prostate cancer. Elife 2017, 6:e27861

46. Arora VK, Schenkein E, Murali R, Subudhi SK, Wongvipat J, Balbas MD, Shah N, Cai L, Efstathiou E, Logothetis C, Zheng D, Sawyers CL: Glucocorticoid receptor confers resistance to antiandrogens by bypassing androgen receptor blockade. Cell 2013, 155: $1309-1322$

47. Shi Z, Fujii K, Kovary KM, Genuth NR, Röst HL, Teruel MN, Barna M: Heterogeneous ribosomes preferentially translate distinct subpools of mRNAs genome-wide. Mol Cell 2017, 67:71-83.e7

48. Arthurs C, Murtaza BN, Thomson C, Dickens K, Henrique R, Patel HRH, Beltran M, Millar M, Thrasivolou C, Ahmed A: Expression of ribosomal proteins in normal and cancerous human prostate tissue. PLoS One 2017, 12:e018604

49. Destefanis F, Manara V, Bellosta P: Myc as a regulator of ribosome biogenesis and cell competition: a link to cancer. Int J Mol Sci 2020, 21:4037

50. Monga J, Subramani D, Bharathan A, Ghosh J: Pharmacological and genetic targeting of 5-lipoxygenase interrupts c-Myc oncogenic signaling and kills enzalutamide-resistant prostate cancer cells svia apoptosis. Sci Rep 2020, 10:6649

51. Coleman DJ, Gao L, King CJ, Schwartzman J, Urrutia J, Sehrawat A, Tayou J, Balter A, Burchard J, Chiotti KE, Derrick DS, Sun D, Xia Z, Heiser LM, Alumkal JJ: BET bromodomain inhibition blocks the function of a critical AR-independent master regulator network in lethal prostate cancer. Oncogene 2019, 38:5658-5669

52. Fan L, Xu S, Zhang F, Cui X, Fazli L, Gleave M, Clark DJ, Yang A, Hussain A, Rassool F, Qi J: Histone demethylase JMJD1A promotes expression of DNA repair factors and radio-resistance of prostate cancer cells. Cell Death Dis 2020, 11:214

53. Santer FR, Höschele PPS, Oh SJ, Erb HHH, Bouchal J, Cavarretta IT, Parson W, Meyers DJ, Cole PA, Culig Z: Inhibition of the acetyltransferases p300 and CBP reveals a targetable function for p300 in the survival and invasion pathways of prostate cancer cell lines. Mol Cancer Ther 2011, 10:1644-1655

54. Aggarwal RR, Schweizer MT, Nanus DM, Pantuck AJ, Heath EI, Campeau E, Attwell S, Norek K, Snyder M, Bauman L, Lakhotia S, Feng FY, Small EJ, Abida W, Alumkal JJ: A Phase Ib/IIa study of the Pan-BET bromodomain inhibitor ZEN-3694 in combination with enzalutamide in patients with metastatic castration-resistant prostate cancer. Clin Cancer Res 2020, 25:5338-5347 
p300 is upregulated by docetaxel and is a target in chemoresistant prostate cancer

Endocr Relat Cancer. 2020 March 27.

doi: 10.1530/ERC-19-0488

This is an open access article distributed under the terms of the Creative Commons CC-BY license, which permits unrestricted use, distribution, and reproduction in any medium, provided the original work is properly cited. 


\title{
p300 is upregulated by docetaxel and is a target in chemoresistant prostate cancer
}

\author{
Martina Gruber1, Lavinia Ferrone1,2, Martin Puhr1, Frédéric R Santer', Tobias Furlan1, Iris E Eder', Natalie Sampson'1, \\ Georg Schäfer ${ }^{3}$, Florian Handle ${ }^{1,4}$ and Zoran Culig1
}

1Division of Experimental Urology, Department of Urology, Medical University of Innsbruck, Innsbruck, Austria 2Department of Biomedical, Experimental and Clinical Sciences, University of Florence, Florence, Italy

3Department of Pathology, Neuropathology, and Molecular Pathology, Medical University of Innsbruck, Innsbruck, Austria

${ }^{4}$ Molecular Endocrinology Laboratory, Department of Cellular and Molecular Medicine, KU Leuven, Leuven, Belgium

Correspondence should be addressed to Z Culig: zoran.culig@i-med.ac.at

\begin{abstract}
Administration of the microtubule inhibitor docetaxel is a common treatment for metastatic castration-resistant prostate cancer (mCRPC) and results in prolonged patient overall survival. Usually, after a short period of time chemotherapy resistance emerges and there is urgent need to find new therapeutic targets to overcome therapy resistance. The lysine-acetyltransferase p300 has been correlated to prostate cancer (PCa) progression. Here, we aimed to clarify a possible function of p300 in chemotherapy resistance and verify p300 as a target in chemoresistant PCa. Immunohistochemistry staining of tissue samples revealed significantly higher p300 protein expression in patients who received docetaxel as a neoadjuvant therapy compared to control patients. Elevated p300 expression was confirmed by analysis of publicly available patient data, where significantly higher p300 mRNA expression was found in tissue of mCRPC tumors of docetaxel-treated patients. Consistently, docetaxel-resistant PCa cells showed increased p300 protein expression compared to docetaxel-sensitive counterparts. Docetaxel treatment of PCa cells for $72 \mathrm{~h}$ resulted in elevated $\mathrm{p} 300$ expression. shRNAmediated p300 knockdown did not alter colony formation efficiency in docetaxelsensitive cells, but significantly reduced clonogenic potential of docetaxel-resistant cells. Downregulation of p300 in docetaxel-resistant cells also impaired cell migration and invasion. Taken together, we showed that p300 is upregulated by docetaxel, and our findings suggest that p300 is a possible co-target in treatment of chemoresistant PCa.
\end{abstract}

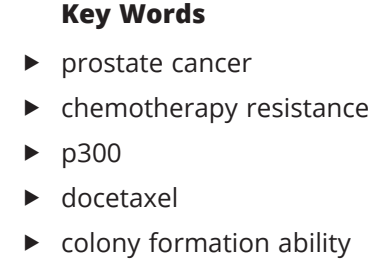

Key Words

- prostate cancer

- chemotherapy resistance

- p300

- colony formation ability

\section{Introduction}

Although therapy of organ-confined prostate cancer (PCa) by radical prostatectomy or radiotherapy is curative, treatment of advanced PCa is considered merely palliative. Androgen deprivation therapy (ADT) remains the gold standard for patients with prostate-specific antigen (PSA) progression. However, tumor progression is inevitable and leads to the development of castration-resistant prostate cancer (CRPC). Treatment options for non-metastatic and metastatic CRPC (mCRPC) include inhibitors of androgen synthesis and antiandrogens such as enzalutamide and apalutamide as well as the chemotherapeutic compound docetaxel (Taxotere $\left.{ }^{\circledR}\right)$.

In 2004, the landmark study TAX327 showed a significant survival benefit of 2.4 months for docetaxel https://erc.bioscientifica.com

https://doi.org/10.1530/ERC-19-0488 (c) 2020 The authors Published by Bioscientifica Ltd. Printed in Great Britain

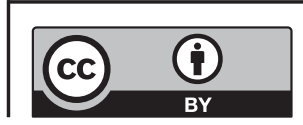

This work is licensed under a Creative Commons Attribution 4.0 International License.
Endocrine-Related Cance (2020) 27, 187-198 
treatment compared to mitoxantrone, which was the first study to demonstrate a survival benefit for chemotherapy in CRPC patients (Tannock et al. 2004). Docetaxel treatment resulted in PSA decline, prolonged overall survival (OS), and improved quality of life. Furthermore, the STAMPEDE and CHAARTED trials have utilized docetaxel together with ADT into first-line treatment for hormone sensitive PCa (HSPC) with a survival benefit of 13.4 months compared to ADT alone (James et al. 2016, Kyriakopoulos et al. 2018). Additionally, docetaxel treatment has no negative consequences for subsequent endocrine therapies. Both abiraterone acetate and enzalutamide are used as effective second-line therapies after resistance to docetaxel has evolved (Lavaud et al. 2018). Despite development of novel therapies, treatment options for mCRPC patients are still limited, and there is an urgent need to find new therapeutic targets to overcome chemotherapy resistance.

Transcriptional co-regulators of the AR are involved in therapy resistance with several of them increasingly expressed during ADT (Comuzzi et al. 2004, Heemers et al. 2007, Qin et al. 2014). Two of these well-known coactivators are the histone acetyltransferases p300 and CBP (CREB binding protein) that show elevated expression in advanced PCa and have oncogenic potential (Debes et al. 2003, Comuzzi et al. 2004). While these coactivators show high levels of homology, they play distinctive roles in PCa and other diseases (Kalkhoven 2004, Ramos et al. 2010). Common features of p300 and CBP include regulation of transcription via remodeling chromatin structure by acetylating conserved lysine amino acids of histone proteins. They are also capable of recruiting the basal transcription machinery to gene promoters and acting as adaptor molecules (Chan \& La Thangue 2001). In a previous study, it was demonstrated that p300 might be a valid target in PCa cells, as downregulation of p300 induced apoptosis and decreased cell migration in androgen-dependent and CRPC cells (Santer et al. 2011). Based on those previous results, this study aimed to investigate whether p300 is a possible new target in the context of chemotherapy resistance. Therefore, we analyzed (1) p300 expression in patients that received docetaxel, (2) p300 expression in docetaxelresistant (DR) cells compared to docetaxel-sensitive counterparts, and (3) the effects of short-term docetaxel treatment on p300 expression. To study the functional role of p300, an RNA-interference (RNAi) approach was used and doxycycline-inducible p300 knockdown cell lines were generated. In addition, effects of p300 downregulation on colony formation efficiency, cell migration, and invasion in docetaxel-resistant cells were determined.

\section{Materials and methods}

\section{Cell culture and chemicals}

Human PCa cell lines PC3 and DU145 were purchased from the American Type Culture Collection (ATCC, LGC Standards, Wesel, Germany). Docetaxel-resistant PC3-DR and DU145-DR were previously established by Puhr et al. (2012). CWR22RV1 and CWR22RV1-DR cells were a kind gift of Prof Dr William Watson (University College Dublin). All cell lines were cultured in RPMI 1640 (PAN Biotech, Aidenbach, Germany) supplemented with $10 \%$ (v/v) fetal bovine serum (PAN Biotech, Aidenbach, Germany), $1 \%(\mathrm{v} / \mathrm{v})$ penicillin/streptomycin, and 1\% (v/v) GlutaMAX (both from Lonza, Vienna, Austria). Docetaxel-resistant cell lines were cultured in the presence of $12.5 \mathrm{nmol} / \mathrm{L}$ docetaxel (Sigma Aldrich). HEK293FT cells were obtained from Life Technologies and grown according to the manufacturer's instructions. The authenticity of all cell lines was validated via short tandem repeat (STR) profiling.

\section{Immunohistochemistry (IHC)}

For IHC staining, a tissue microarray (TMA) of 14 patients that received neoadjuvant docetaxel therapy before radical prostatectomy and 14 patients with no chemotherapy was used. The use of patient material was approved by the Ethics Committee of the Medical University of Innsbruck (study No AM 3174 including amendment 2). For detailed information about clinical data from patients, see publication of Puhr et al. (2012). IHC staining was performed on a Discovery-XT staining device (Ventana) and the following specific antibody was used: anti-p300 (1:100, D8Z4E, Cell Signaling Technology). Antibody specificity was verified by Western blot and IHC staining of PC3-DR cells with p300 downregulation. For IHC, cells were embedded by coagulation in plasma clots after harvesting, transferred into a biopsy histosette, fixed in formalin, and embedded in paraffin. Importantly, cross staining of CBP was excluded by Western blot analysis.

\section{Transcriptome analysis of patient data}

The publicly available transcriptome dataset GSE77930 (Kumar et al. 2016) was downloaded from the GEO database and analyzed with the Qlucore Omics Explorer v3.5. Gene set activity scores for the Hallmark 'Androgen response' and 'Myc targets' gene sets (Molecular Signatures Database, MSigDB) were calculated in $\mathrm{R}$ with the GSVA package (Hanzelmann et al. 2013).

This work is licensed under a Creative Commons Attribution 4.0 International License. 


\section{Western blot}

Cells were lysed in LDS sample buffer and $50 \mu \mathrm{g}$ total protein was separated either on 3-8\% Tris-Acetate gels (Thermo Fisher Scientific) for analysis of p300 and CBP expression or $4-12 \%$ Bis-Tris gels (Expedeon, San Diego, CA, USA) for all other proteins and transferred onto methanol-activated PVDF membranes or $0.2 \mu \mathrm{m}$ nitrocellulose membranes (GE Healthcare). Blocking of membranes and antibody incubation were performed in 5\% BSA in TBS-T. The following antibodies were used: anti-p300 (1:4000, ab10485, Abcam), anti-CBP (1:1000, Cell Signaling Technology), antic-Myc (1:1000, Cell Signaling Technology), anti-Histone H3 (1:1000, Cell Signaling Technology), anti-Acetyl-Histone H3 (Lys18, 1:1000, Cell Signaling Technology), anti-Vimentin (1:500, Santa Cruz Biotechnology), anti-Vinculin (1:500, Santa Cruz Biotechnology), anti-Lamin A (1:2000, Abcam), anti- $\alpha$-tubulin (1:500, Santa Cruz Biotechnology), and antiGAPDH (1:50000, Merck Millipore). House-keeping controls were selected in a cell line-specific manner on the basis of data showing no change in their expression in that cell line.

\section{RNA isolation and quantitative real-time PCR}

Total RNA was isolated using the EXTRACTME TOTAL RNA KIT (LabConsulting, Vienna, Austria) according to the manufacturer's manual. cDNA synthesis was performed with the iScript Select cDNA Synthesis Kit (Bio-Rad). For real-time PCR a Luna Script RT Super Mix Kit (New England Biolabs, Ipswich, MA, USA) was used. HPRT1 (Fwd: GCTTTCCTTGGTCAGGCAGTA, Rev: GTCTGGCTTATATCCAACACTTCGT, Probe: CAAGGTCGCAAGCTTGCTGGTGAAAAGGA), TATA-Box binding protein (TBP; Fwd: CACGAACCACGGCACTGATT, Rev: TTTTCTTGCTGCCAGTCTGGAC, Probe: TCTTCACTCTTGGCTCCTGTGCACA), and HMBS (TaqMan Gene Expression Assay from Thermo Fisher Scientific; Hs00609297_m1) were used as reference genes. The following Taqman gene expression assays were used: p300 (Hs00914223_m1), CBP (Hs00932878_m1), c-Myc (Hs00153408_m1), and Vimentin (Hs00185584_m1).

\section{Generation of doxycycline-inducible knockdown cell lines}

Stable cell lines with inducible p300 knockdown were generated by lentiviral-based transduction of shRNA vectors using the BLOCK-iT HiPerform Lentiviral Pol-II miR RNAi Expression System with emGFP from Invitrogen. Briefly, miR Select oligos (Hmi405238: shp300-1;
Hmi405239: shp300-2) were purchased from Life Technologies and ligated into pcDNA 6.2-GW/EmGFPmiR expression vector according to the manufacturer's protocol. Then the shRNAs were shuttled into the pDONR221 vector to generate entry clones. Entry plasmids together with a pENTR-tetOn (from pHR-TetCMV-eGFPdest ligated into pENTR 5'/CMVp vector) were then used in MultiSite Gateway reactions with pLenti6.4/R4R2/ V5-DEST to generate doxycycline-inducible shp300-1 and shp300-2. To generate cell lines stably expressing the doxycycline activator/repressor cassette, HEK293FT cells were first co-transfected with the packaging vectors pVSV-G and psPAX2 together with pHR-SFFV-rtTAM2T2A-Puro using X-tremeGENE reagent (Roche) according to the manufacturer's manual. Supernatants containing virus particles were collected $48 \mathrm{~h}$ after transfection, filtered through a $0.45 \mu \mathrm{m}$ membrane filter, and used for infection of target cells. Cells were selected with $2 \mu \mathrm{g} / \mathrm{mL}$ puromycin and subsequently transfected with packaging vectors together with the pLenti6.4 expression vector as described previously. Selection of infected cells was performed using $2 \mu \mathrm{g} / \mathrm{mL}$ blasticidine. For activation of the system, $100 \mathrm{ng} / \mathrm{mL}$ doxycycline was used. Activation status of the system was verified by GFP expression.

\section{Proliferation assay}

Cumulative population doubling levels (PDL) were determined by continuously seeding a defined number of cells in T25 flasks. Cell numbers were determined by CASY cell counter (Schärfe System, Reutlingen, Germany) every 3-4 days. Cumulative PDL was calculated with the following formula: $\mathrm{PDL}=3.32 \times(\log 10(\mathrm{X})-\log 10(\mathrm{Y}))+\mathrm{I}$, where $\mathrm{X}=$ number of cells at the end of growth period, $\mathrm{Y}=$ number of cells at the beginning of growth period, and $\mathrm{I}=$ initial population doubling level. To compare the growth curves, linear fit regression with the test for significance of slopes and intercepts was performed in GraphPad Prism 8.

\section{High-resolution colony formation assay}

Cell numbers were determined using CASY cell counter system (Schärfe System). Per $75 \mathrm{~cm}^{2}$ culture flask, 1000 cells were seeded and incubated for 10-14 days in the absence or presence of $100 \mathrm{ng} / \mathrm{mL}$ doxycycline. Cells were fixed with 100\% ice-cold methanol for 5 min and stained with crystal violet $(0.5 \%$ dissolved in PBS containing 20\% methanol (Sigma)) for $5 \mathrm{~min}$. Colony formation efficiency was determined by the software 
CATCH-colonies (https://catch-colonies.net). To correct for differences in the seeding density of the different stable shRNA cell lines, the colony formation efficiency was normalized to flasks that were seeded at the same time but not treated with doxycycline.

\section{Wound scratch assay}

Cells were seeded until they were nearly confluent in multiwell plates and treated with $100 \mathrm{ng} / \mathrm{mL}$ doxycycline or 10 HM CPI-637 (MedChem Express, Monmouth Junction, NJ, USA) for $96 \mathrm{~h}$ in total. After the first $24 \mathrm{~h}, 10 \mu \mathrm{M}$ of the proliferation inhibitor cytosine $\beta$-D-arabinofuranoside was added. After another $24 \mathrm{~h}$, a scratch was made using a 10- $\mu \mathrm{L}$ pipette tip. Images were taken after another $48 \mathrm{~h}$ and analyzed using the MRI Wound Healing Tool of ImageJ.

\section{Migration and invasion assay}

For migration and invasion assays, Boyden chamber inserts with $8 \mu \mathrm{m}$ pore size (Fluoroblok System, Becton Dickinson) were used. $3 \times 10^{4}$ cells per well of PC3-DR shCtrl, PC3-DR shp300-1, and PC3-DR shp300-2 were seeded in duplicate in serum-free medium. Bottom chambers were filled with medium containing 10\% FCS serving as chemoattractant. For invasion assays, inserts were coated with $30 \mu \mathrm{l}$ of matrigel (diluted 1:3 in serumfree medium; Corning). Cells were incubated for $48-72 \mathrm{~h}$ in the absence or presence of $100 \mathrm{ng} / \mathrm{mL}$ doxycycline or $20 \mu \mathrm{M}$ CPI-637, respectively. Afterwards, cells were stained with $2 \mu \mathrm{M}$ of calcein AM (Sigma) diluted in HBSS containing 1\% FCS. Fluorescent images were taken using a JuLI smart fluorescent cell analyzer (Science Services, Munich, Germany), and extinction/emission at 494/517 was measured using TECAN plate reader (Tecan Group Ltd., Männedorf, Switzerland).

\section{Immunofluorescence}

Cells were seeded on glass coverslips in the absence or presence of $100 \mathrm{ng} / \mathrm{mL}$ doxycycline for $72 \mathrm{~h}$. Cells were fixed with $4 \%$ paraformaldehyde and permeabilized with 1\% BSA in PBS containing 0.2\% Triton X-100. Washing steps were performed with 1\% BSA in PBS. AntiVimentin (1:500, Santa Cruz Biotechnology) was used as a primary antibody, together with the fluorescence-labeled secondary antibody goat anti-mouse 555 (ThermoFisher). Coverslips were mounted with Vectashield mounting medium containing DAPI.

\section{Viability assays}

PC3-DR cells were seeded in multi-well plates and treated with a range of CPI-637 concentrations. The solvent DMSO served as a control. The viability was measured using RealTime-Glo ${ }^{\text {TM }}$ MT Cell Viability Assay (Promega) after $72 \mathrm{~h}$ and the IC50 was calculated using a nonlinear fit model with variable slope of log transformed data in GraphPad Prism 8.

\section{Statistical analysis}

Statistical analysis was performed using GraphPad Prism 8 (GraphPad Software Inc.). Gaussian distribution of patient samples was determined using Kolmogorov-Smirnov test. Comparison of the two groups was performed using Student's $t$-test and Mann-Whitney $U$ test, depending on Gaussian distribution. Comparison of multiple groups was performed using one-way ANOVA and correcting for multiple testing using Bonferroni multiple comparison test. For integration of multiple independent replicates from methods that yield relative abundance values (qPCR, Western blot), each replicate was normalized to its average signal intensity. $P$-values of $<0.05$ were considered statistically significant and encoded in figure legends as follows: ${ }^{*} P<0.05 ;{ }^{* *} P<0.01 ;{ }^{* *} P<0.001$; and ${ }^{* * * *} P<0.0001$. If not stated otherwise, doxycycline-treated cells were normalized to non-treated control cells, and doxycycline-treated cells are shown in graphs.

\section{Results}

\section{p300 expression is increased in docetaxel-} treated patients

To evaluate whether p300 expression is altered upon docetaxel treatment, we analyzed tissue material of patients who received docetaxel by IHC staining using a specific p300 antibody (Supplementary Fig. 1A, B and $\mathrm{C}$, see section on supplementary materials given at the end of this article). In total, 28 patients were included, 14 of them received neoadjuvant docetaxel therapy before radical prostatectomy (RPE), while the other 14 patients did not receive chemotherapy prior to RPE. For detailed information about selected patients, please see the work of Puhr et al. (2012), where clinical data are fully described. We observed a significantly increased p300 expression in cancerous areas of docetaxel-treated patients compared to control patients (Fig. 1A and B), which was not the case in benign areas. Furthermore, p300 staining was observed mainly in nuclear areas.

This work is licensed under a Creative Commons Attribution 4.0 International License. 
Elevated p300 expression was further confirmed in publicly available datasets of mCRPC tissue samples from patients that suffered from relapse after docetaxel treatment (Kumar et al. 2016). Our analysis revealed significantly increased p300 levels (1.5-fold) in patients treated with docetaxel compared to patients that did not receive docetaxel at any time in their treatment course (Fig. 1C). To assess if docetaxel-mediated increase is specific for p300, we included the analysis of the closely related coactivator CBP. Interestingly, CBP expression levels were found slightly decreased in docetaxel-treated patients compared to non-docetaxel-treated patients (Fig. 1C). Furthermore, we analyzed AR expression and activity upon docetaxel treatment to evaluate whether the AR has any impact on docetaxel-mediated upregulation of $\mathrm{p} 300$. Of note, AR mRNA expression and androgen response were not significantly changed in patients that relapsed after docetaxel treatment compared to control patients (Supplementary Fig. 2A and B).

\section{Docetaxel-resistant and docetaxel-treated PCa cells show increased p300 expression}

Next, we compared p300 expression in docetaxelresistant (DR) derivatives of commonly employed PCa cell lines (PC3-DR, DU145-DR, and CWR22RV1-DR) relative to their docetaxel-sensitive counterparts. p300 protein levels were significantly increased (1.5-fold) in all three docetaxel-resistant cell lines tested compared to docetaxel-sensitive counterparts (Fig. 2A), whereas mRNA levels were unchanged (Supplementary Fig. 3A). Increased p300 protein expression in docetaxel-resistant PC3-DR and DU145-DR was confirmed by IHC staining (Fig. 2B). CBP mRNA and protein expression were not significantly regulated in docetaxel-resistant cells compared to sensitive counterparts (except downregulated CBP mRNA expression in PC3-DR cells, Supplementary Fig. 3B and C).

To understand the molecular basis of these findings, we analyzed p300 expression upon short-term docetaxel treatment in several PCa cell lines. To this end, we measured p300 mRNA and protein expression in PC3, DU145, and CWR22RV1 cells after treatment with docetaxel for $72 \mathrm{~h}$. Protein expression of p300 significantly increased (2-2.5-fold increase) in all three cell lines upon docetaxel treatment (Fig. 2C), whereas mRNA levels were not significantly changed (Supplementary Fig. 4A). Time-course experiments revealed that $\mathrm{p} 300$ protein, but not mRNA expression, increased within $8 \mathrm{~h}$ of docetaxel treatment and reached a plateau after $16 \mathrm{~h}$ (Supplementary Fig. 4B and C). Interestingly, p300 expression in docetaxelresistant cells decreased if the cells were cultured without docetaxel (Supplementary Fig. 4D and E). To investigate
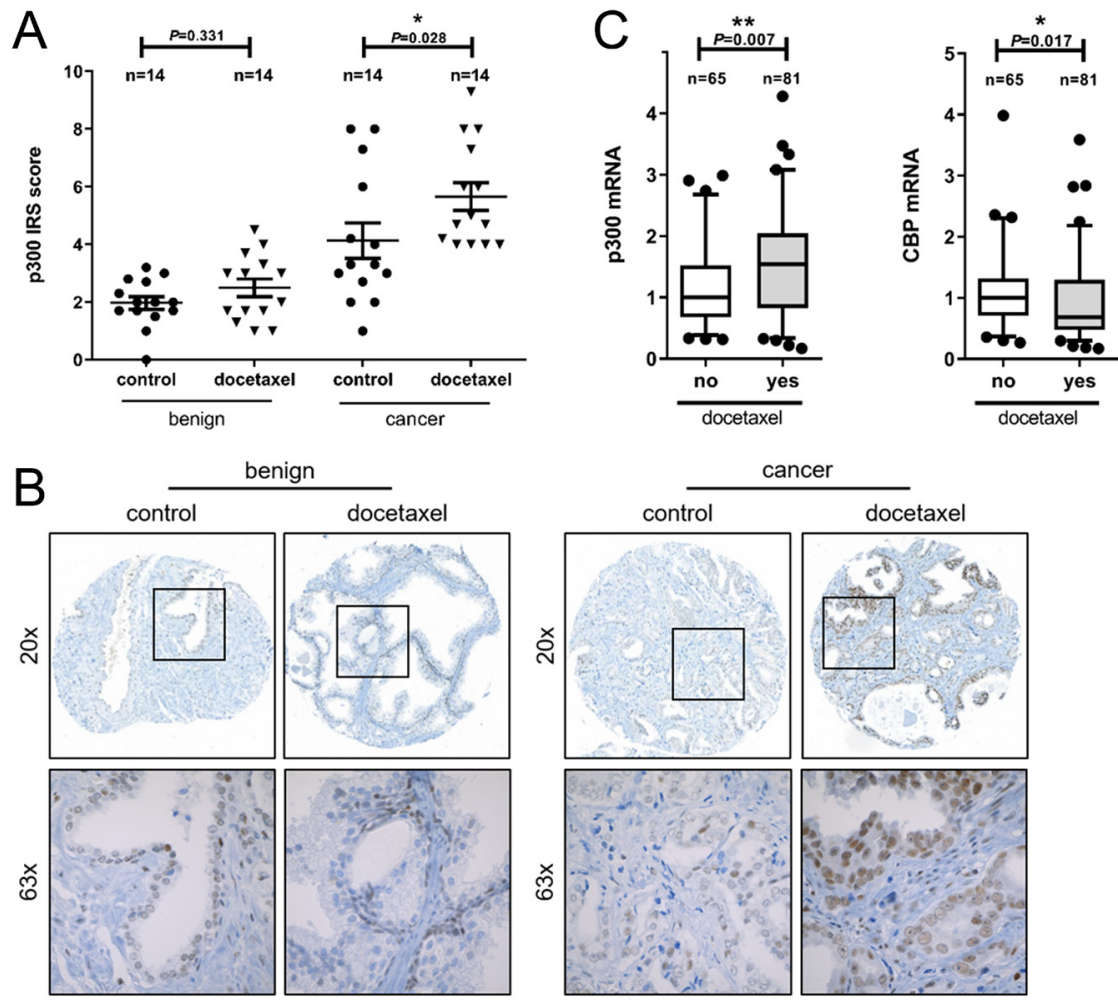

Figure 1

Expression of p300 is increased upon docetaxel treatment. (A) Quantification of p300 immunoreactivity scores (IRS) after IHC staining (Mann-Whitney $U$ test; scatter dot blot with line at mean + S.E.M.). (B) IHC staining of p300 (nuclear localization). Upregulation of p300 expression in cancerous tissue of docetaxel-treated patients compared to control patients. (C) p300 and CBP mRNA expression were determined in samples of docetaxel-treated and docetaxel-untreated mCRPC patients (Mann-Whitney $U$ test; box whisker plot with 5-95 percentile). https://erc.bioscientifica.com https://doi.org/10.1530/ERC-19-0488 (c) 2020 The authors Published by Bioscientifica Ltd. Printed in Great Britain
This work is licensed under a Creative Commons Attribution 4.0 International License. 
A

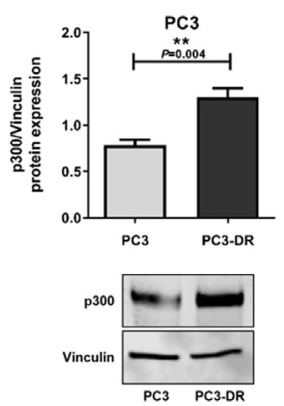

B

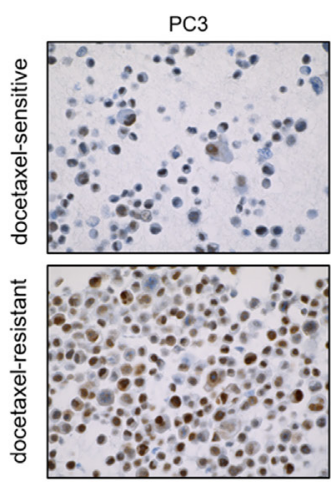

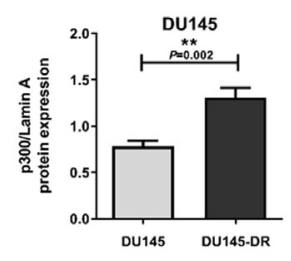
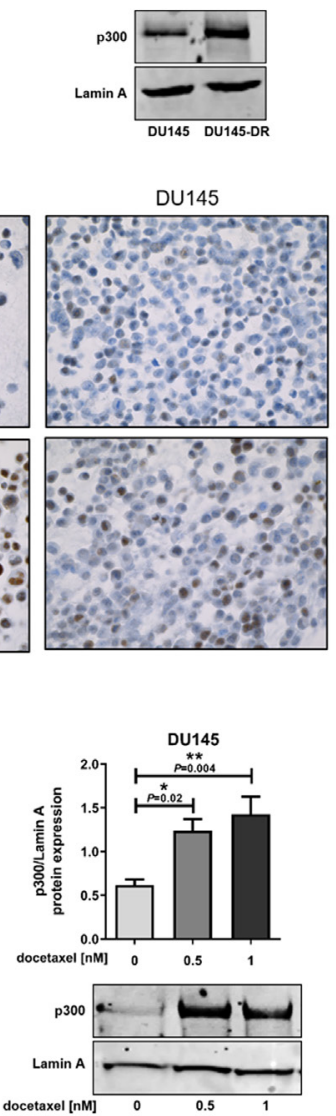
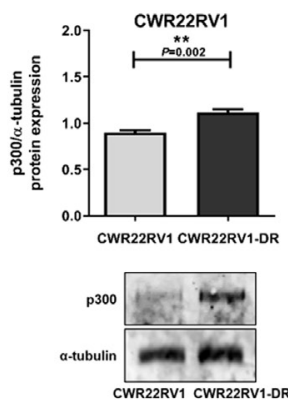

CWR22RV1
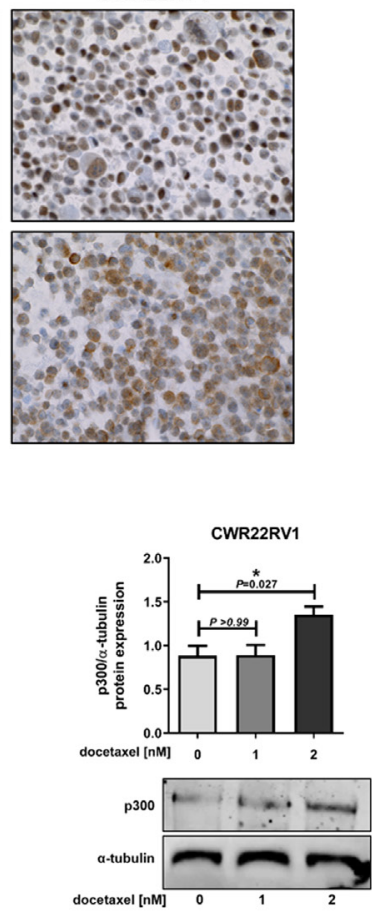

Figure 2

p300 expression is increased in docetaxelresistant prostate cancer cells. (A) Comparison of p300 protein expression between docetaxelsensitive and docetaxel-resistant (DR) PC3 $(n=4)$, DU145 $(n=5)$, and CWR22RV1 $(n=4)$. Data represent mean + S.E.M. (t-test). (B) IHC staining for p300 in docetaxel-resistant PC3-DR, DU145-DR, and CWR22RV1-DR compared to docetaxelsensitive counterparts. Magnification 40×. (C) PC3 $(n=5), \operatorname{DU} 145(n=5)$, and CWR22RV1 $(n=4)$ were treated with the indicated concentrations of docetaxel for $72 \mathrm{~h}$, and p300 protein expression was analyzed by Western blot, and one representative Western blot is shown. Values represent mean + S.E.M. (one-way ANOVA). whether docetaxel influences the protein degradation rate of p300, translation was blocked with cycloheximide (CHX). Of note, p300 had a protein half-life time of around $6 \mathrm{~h}$ in untreated PC3 cells, whereas addition of docetaxel (PC3 and PC3-DR) stabilized the protein (Supplementary Fig. 4F).

We also analyzed the expression levels of the welldescribed p300 downstream target c-Myc, which is a known oncogene in PCa (Koh et al. 2010). Our analysis revealed no significant change of c-Myc mRNA levels (Supplementary Fig. 5A), but a Myc gene expression activity score was significantly increased in docetaxel-treated patients (Supplementary Fig. 5B). Additionally, docetaxel-resistant PC3-DR and DU145-DR showed higher c-Myc protein expression compared to docetaxel-sensitive counterparts (Supplementary Fig. 5C), and c-Myc protein expression was increased (1.5-fold) in DU145 upon treatment with $1 \mathrm{nM}$ docetaxel compared to the control (Supplementary Fig. 5D).

\section{Docetaxel-resistant cells show reduced colony formation ability upon p300 inhibition}

To study a possible mechanistic role of p300 in docetaxel resistance, PC3, CWR22RV1, and their docetaxel-resistant counterparts were stably transduced with doxycyclineinducible short hairpin (sh)p300 vectors. We selected these cell lines to include both AR-negative (PC3 and PC3-DR) as well as AR-positive (CWR22RV1 and CWR22RV1-DR) sublines. Doxycycline treatment activated the inducible system as indicated by homogeneous expression of vector-integrated GFP (Fig. 3A), which led to decreased p300 protein expression (Fig. 3B) and activity by decreased acetylation of histone h3 on lysine 18 (Fig. 3C). Of note, p300 knockdown had no biologically consistent effect on the proliferation rate of docetaxelsensitive or docetaxel-resistant cells over a period of 20 days (Fig. 3D). The significantly reduced proliferation https://erc.bioscientifica.com https://doi.org/10.1530/ERC-19-0488
(C) 2020 The authors Published by Bioscientifica Ltd. Printed in Great Britain
This work is licensed under a Creative Commons Attribution 4.0 International License.

Downloaded from Bioscientifica.com at 04/13/2021 12:27:30PM 
rate of PC3-DR shp300-2 was not reproducible with the shp300-1 construct and is, thus, likely an artifact or off-target effect. To exclude the possibility that CBP is upregulated by p300 inhibition, thus compensating for the effects of p300 downregulation, we analyzed CBP expression upon p300 knockdown. As expected, specific downregulation of p300 had no impact on CBP expression (Supplementary Fig. 6).

An important characteristic of aggressive tumor cells is the ability to form colonies, thereby assessing a single cell's ability to undergo unlimited division. In docetaxel-sensitive PC3 and CWR22RV1, knockdown of p300 had no significant effect on the clonogenic potential (Fig. 4A). However, interestingly, p300 inhibition in docetaxel-resistant PC3-DR and CWR22RV1-DR significantly reduced colony formation efficiency (reduction by 40-50\% in PC3-DR and 20-30\% in CWR22RV1-DR; Fig. 4B).

\section{Cell migration and invasion are impaired upon p300 downregulation in docetaxel-resistant cells}

It has previously been shown that p300 is involved in migration and invasion in PCa cells (Santer et al. 2011). Therefore, we wanted to test if p300 inhibition might also impair cell migration and invasion in docetaxel-resistant cells. We employed PC3-DR cells for these experiments since the migration ability of CWR22RV1-DR cells is low. We first performed wound scratch assays that revealed a 2.5-fold decrease in the wound healing rate upon p300 downregulation (Fig. 5A). Consistently, significantly decreased cell migration by $~ 50 \%$ was observed in Boyden chambers following p300 downregulation (Fig. 5B). Moreover, invasion assays, which were conducted on matrigel-coated membranes, also showed a significantly reduced invasion ability (reduction by 50\%) upon p300 downregulation (Fig. 5C). These findings were confirmed
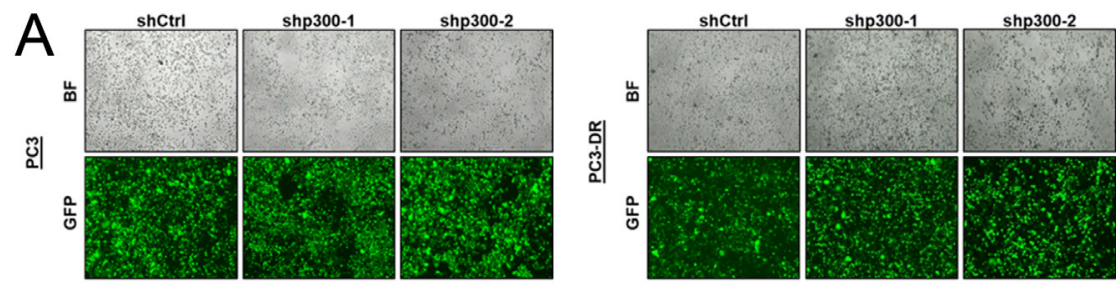

B
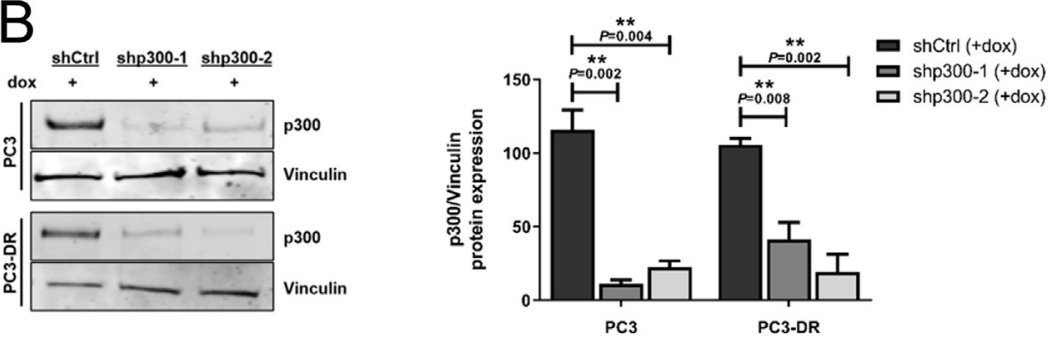

C

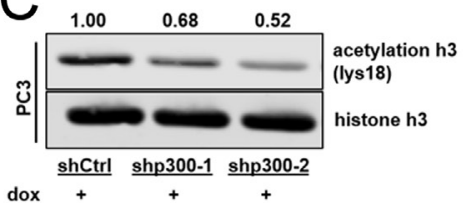

$\mathrm{D}$

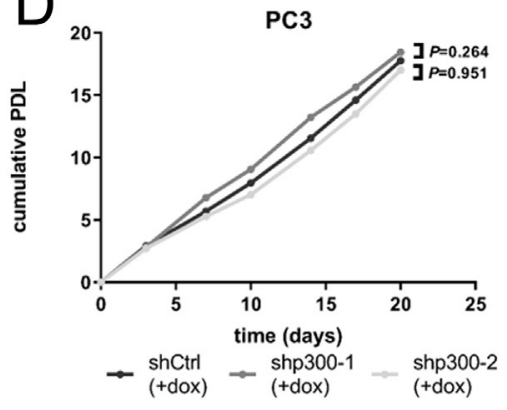

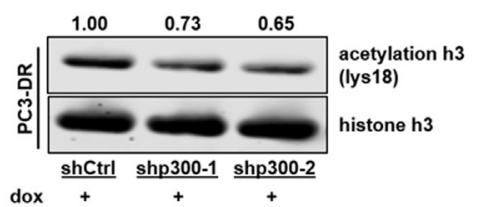

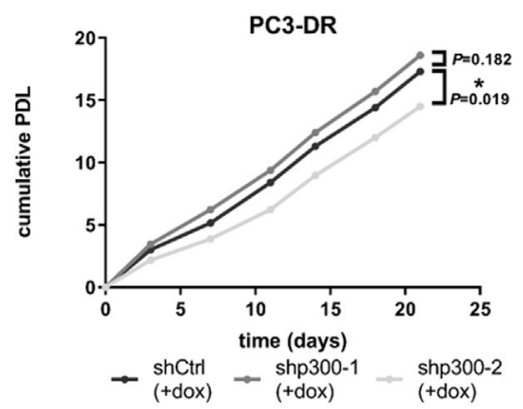

Figure 3

Establishment and validation of doxycyclineinducible p300 knockdown cell lines using a non-targeting control (shCtrl) sequence and two specific p300-shRNA sequences (shp300-1 and shp300-2). Docetaxel-sensitive PC3 and docetaxel-resistant PC3-DR are shown here, representing all mentioned cell lines. For better visualization in the following experiments, only doxycycline-treated shCtrl, shp300-1, and shp300-2 replicates are shown. Confirmation of (A) uniform expression of shRNA-constructs by fluorescence microscopy ( $\mathrm{BF}=$ bright field, GFP = green fluorescent protein) and magnification 40x and (B) p300 knockdown by Western blot analysis after activation of shp300-sequences with $100 \mathrm{ng} / \mathrm{mL}$ doxycycline for $72 \mathrm{~h}$. Values indicated are mean + S.E.M. (one-way ANOVA, $n=3$ ). (C) Decreased p300 activity was confirmed by analysis of histone h3 acetylation on lysine18 in PC3 and PC3-DR cells. One representative Western blot out of three independent experiments is shown. (D) Cumulative population doubling levels (PDL, $n=1$ ) of PC3 and PC3-DR cells over time with downregulated $\mathrm{p} 300$ were calculated by cell number measurement upon each passage (linear fit regression and test for significance of slopes and intercepts). https://erc.bioscientifica.com https://doi.org/10.1530/ERC-19-0488 Printed in Great Britain

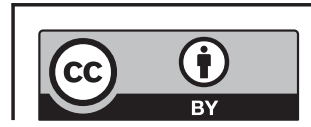

This work is licensed under a Creative Commons Attribution 4.0 International License. 

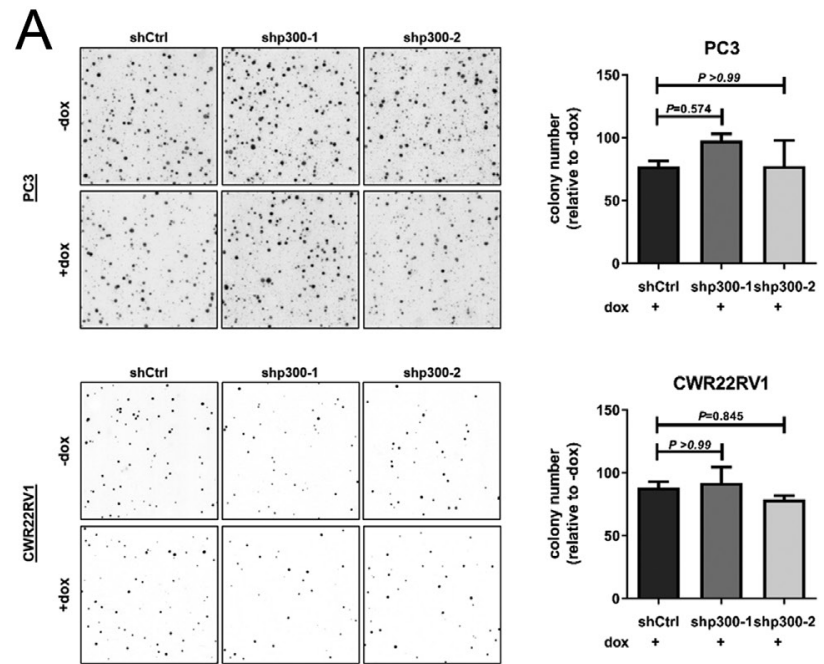
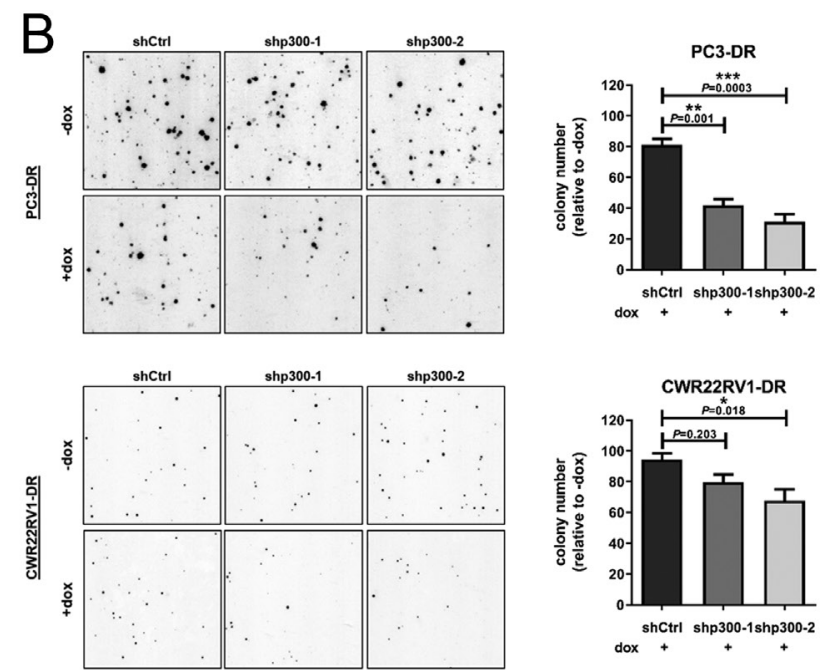

Figure 4

p300 inhibition decreases colony formation ability of docetaxel-resistant cells. Representative images and quantification of high-resolution colony formation assays of docetaxel-sensitive PC3 and CWR22RV1 (A) and docetaxel-resistant PC3-DR and CWR22RV1-DR (B). Colony numbers were analyzed by the software CATCH-colonies. Data represent mean + S.E.M. (one-way ANOVA, $n=3$ ).

by the use of the dual inhibitor of p300 and CBP CPI637. Viability assays were performed to calculate the IC50 for PC3-DR cells (Supplementary Fig. 7). It was determined that IC50 for CPI-637 is $17.52 \mu \mathrm{M}$. PC3-DR cells treated with CPI-637 for $72 \mathrm{~h}$ revealed a significantly decreased migration and invasion ability (reduction by $50-60 \%$, Fig. 5D, E and F). Vimentin is a cytoskeleton component that plays an important role in migration and was therefore analyzed in p300-silenced PC3-DR cells by immunofluorescence, where we observed a significantly decreased vimentin protein expression (by 60-70\%, Fig. 5G). Reduced vimentin levels were additionally confirmed by qRT-PCR and Western blot analysis (Fig. 5H).

\section{Discussion}

Although chemotherapy for CRPC patients confers a clinical benefit for patients, there is no curative treatment available for late stages of PCa. Development of chemotherapy resistance occurs rapidly, and it remains largely unclear which factors are differentially expressed during chemotherapy and might contribute to docetaxel insensitivity. Thus, it is important to understand the molecular mechanisms and to find new therapeutic targets to overcome therapy failure.

As a coactivator of the AR, p300 is involved in many biological processes such as differentiation, proliferation, and cell cycle regulation (Iyer et al. 2004) and has already been associated with tumor progression and poor prognosis
(Debes et al. 2003). Furthermore, p300 expression is increased upon androgen deprivation (Heemers et al. 2007) and plays an essential role in ligand-independent transactivation of the AR in androgen-independent PCa cells (Debes et al. 2002).

The main finding of this study is that p300 is upregulated upon docetaxel treatment in primary PCa and mCRPC tissue samples as well as in docetaxel-sensitive and docetaxel-resistant PCa cells. IHC staining was performed in samples of patients who received neoadjuvant docetaxel therapy at the Department of Urology of the Medical University of Innsbruck. Of course, these samples do not reflect the same clinical stage as docetaxel-resistant cells. However, patients who receive chemotherapy for PCa in the Authors' institution are not subjected to removal of tumor tissue. Therefore, the samples from individuals who received neoadjuvant docetaxel therapy were selected to be able to analyze p300 expression in patient material. Furthermore, metastatic tissue samples obtained by rapid autopsy from a publicly available transcriptome dataset including $\mathrm{mCRPC}$ patients that received docetaxel in their treatment course were analyzed. Increased 300 expression was observed in vitro in docetaxel-resistant AR-positive and AR-negative cells (PC3-DR and CWR22RV1-DR) compared to their respective docetaxel-sensitive counterparts. This finding indicates that p300 is upregulated by docetaxel regardless of AR expression and is consistent with previous publications documenting multiple functions of p300 in PCa also independently of the AR (Debes et al. 2003, Santer et al. 2011). This is also in concordance with the 
A

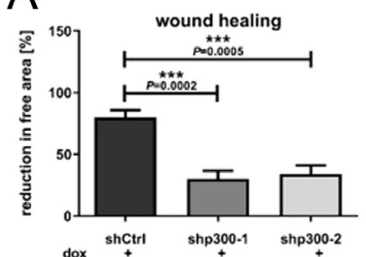

dox shctrl shp300-1 shp300-2

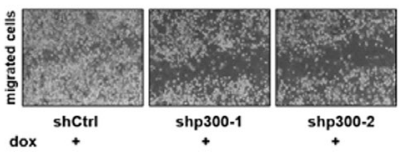

D

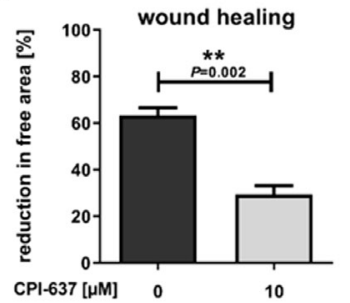

B
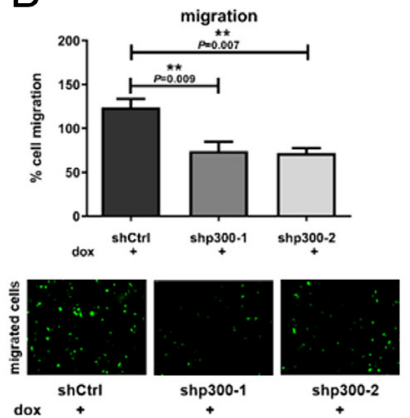

E

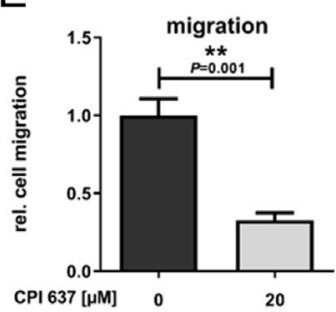

C

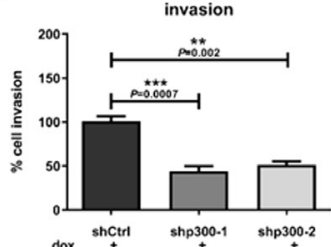

$\underset{\text { shctrl }}{+}$ shp300.1 shp300.2

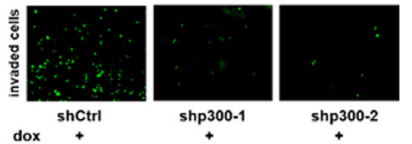

F

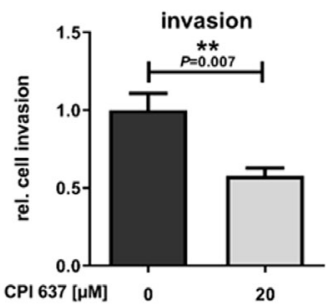

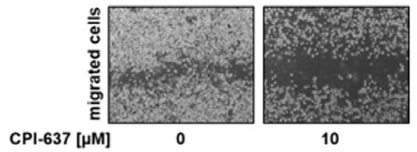

CPI-637 [MM]

10

G

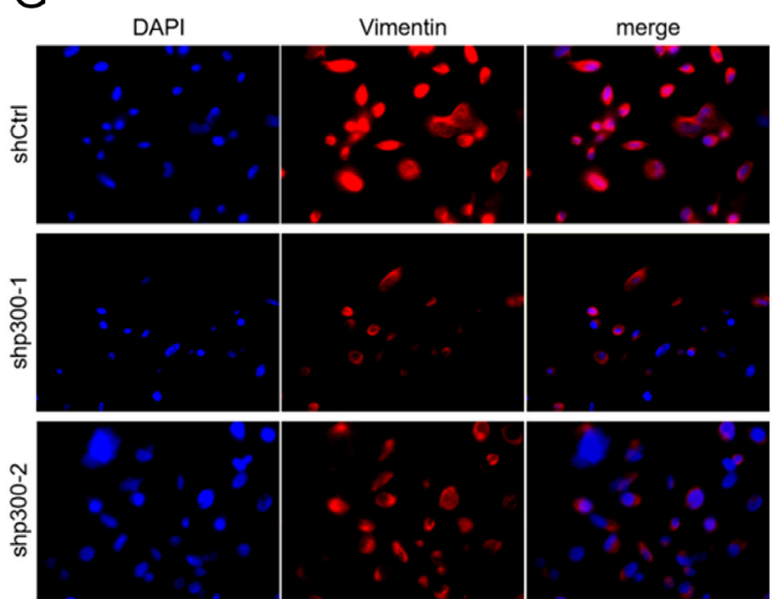

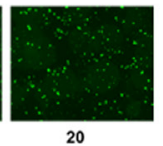

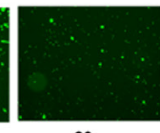

20

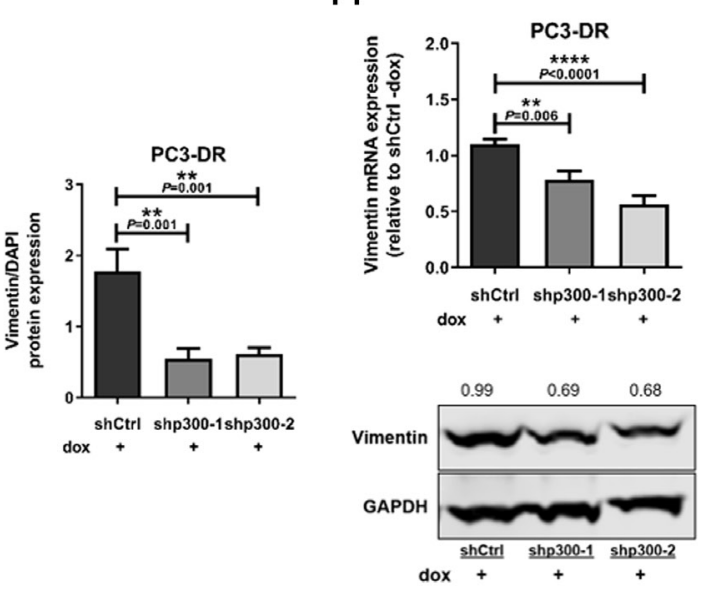

$\mathrm{H}$

Figure 5

p300 downregulation decreases cell migration and invasion. (A) Wound scratch assays were performed on confluent layers of PC3-DR shCtrl, shp300-1, and shp300-2 treated with $100 \mathrm{ng} / \mathrm{mL}$ doxycycline. Images were taken $48 \mathrm{~h}$ after scratch and analyzed by Image $(n=6)$. (B) PC3-DR were seeded in Boyden chambers and shp300 sequences were activated with $100 \mathrm{ng} / \mathrm{mL}$ doxycycline for $72 \mathrm{~h}$. Cell migration was measured after staining with calcein and visualized by fluorescence microscopy $(n=4)$. (C) Invasion assays were conducted as in B, except that Boyden chambers were pre-coated with Matrigel $(n=3)$. Values indicated in A-C denote mean + s.E.M. (one-way ANOVA). (D) Wound scratch assays on PC3-DR treated with $10 \mu \mathrm{M}$ CPI-637. Data represent mean + S.E.M. ( $($-Test, $n=3)$. (E) Migration $(n=4)$ and (F) invasion assays $(n=5)$ of PC3-DR treated with $20 \mu \mathrm{M}$ CPI-637. Values indicated are mean + S.E.M. (t-test). (G) Immunofluorescence staining for vimentin (red) and quantification relative to counterstaining of nuclei (blue). Original magnification 630×. (H) mRNA (qPCR) and protein expression (Western blot) of vimentin. Data in G-H represent mean + S.E.M. (one-way ANOVA, $n=5$ ).

finding that AR expression and activity are not changed upon docetaxel treatment; although, in previous studies it has been shown that docetaxel impairs transcriptional activity of the AR (Zhu et al. 2010). Other groups reported an inhibitory effect of docetaxel on AR activity by interfering with AR intracellular trafficking https://erc.bioscientifica.com https://doi.org/10.1530/ERC-19-0488 (c) 2020 The authors Published by Bioscientifica Ltd. Printed in Great Britain
This work is licensed under a Creative Commons Attribution 4.0 International License. 
(Martin et al. 2015) and nuclear translocation (ThadaniMulero et al. 2014).

Contrary to p300, the closely related coactivator CBP was not upregulated upon docetaxel treatment. Thus, we conclude that docetaxel-induced upregulation is specific for p300. Hatano and colleagues reported a role of c-Myc, which is a known downstream target of p300 in docetaxel resistance (Hatano et al. 2013). Concordant with their study, we observed an increased expression of c-Myc in docetaxel-treated patients; however, the upregulation was not statistically significant. Nevertheless, Myc activity was significantly increased in patients who received docetaxel. Ogiwara and colleagues also reported that p300 ablation caused downregulation of Myc expression in CBPdeficient cells and thereby suppressed cancer cell growth (Ogiwara et al. 2016). We assume that p300 upregulation by docetaxel is not mediated by c-Myc, but c-Myc is affected in consequence as a target gene of p300.

We found that p300 mRNA expression was not significantly changed upon docetaxel treatment, which indicates that p300 protein increase is not due to increased transcriptional activity. p300 protein expression also does not increase because of elevated translation, since p300 protein expression in docetaxel-resistant cells was unchanged upon translation inhibition, which suggests that p300 is protected from proteasomal degradation. In a previous study, it was found that androgens downregulate p300 protein but not mRNA expression (Heemers et al. 2007). Those results suggest similarities in regulation of p300 by docetaxel and androgens.

It has been previously shown that the competitive histone acetyltransferase p300/CBP inhibitor C646 reduced colony formation in AML cell lines and primary blasts (Gao et al. 2013). While this highlights the capacities of HAT inhibitors, there are issues with the potency and selectivity of these early inhibitors (Lasko et al. 2017). Meanwhile, more effective bromodomain and extra-terminal (BET) inhibitors that prevent protein-protein interactions between BET proteins and acetylated histones have been developed. The BET inhibitors INCB054329 and INCB057643 have been shown to be effective as single agents in PCa. Likely, novel BET inhibitors will be combined with existing therapies, in particular, for therapy-resistant PCa (Vazquez et al. 2019). In this study, we initially employed a shRNA approach to down-regulate p300 and exclude non-specific effects. We confirmed effects of shRNA-mediated p300 downregulation on migration ability of docetaxel-resistant cells with the bromodomain inhibitor CPI-637 that is specific for p300 and CBP, where we observed similar results as with genetic p300 downregulation.
In contrast to docetaxel-sensitive cells, p300 inhibition in docetaxel-resistant PC3-DR and CWR22RV1-DR significantly reduced colony formation efficiency. Downregulation of p300 had no specific effects on proliferation of docetaxel-resistant cells, indicating that p300 inhibition indeed impaired the colony-initiating capacity and that the reduced colony number is not just a secondary effect. It has already been described that docetaxel-resistant cells show a stem-cell-like phenotype (Puhr et al. 2012, Marin-Aguilera et al. 2014), suggesting that p300 inhibition is effective in conditions in which pathways connected to colony formation and tumorinitiation play a central role. A possible explanation as to why p300 inhibition shows no effects on colony formation of docetaxel-sensitive cells could be the formation of p300complexes. It has been described that the cysteine protease USP24 stabilizes p300 and thereby increases acetylation of histone h3 (Wang et al. 2018). The CtBP1-p300-FOXO 3a complex was found to repress apoptotic regulators Bax and Bim in osteosarcoma cells (Li et al. 2019). Cell proliferation is regulated by p300 in complex with the transcriptional repressor YY1 and HDAC2 (Tang et al. 2019). Future studies of therapy resistance in PCa should therefore examine and quantitate the complexes relevant to $\mathrm{p} 300$. In contrast to $\mathrm{PCa}$, in some models of breast cancer, p300 is considered a tumor inhibitor (Asaduzzaman et al. 2017). However, the histone methyltransferase DOT1L in complex with p300 and c-Myc enhanced cellular stemness (Cho et al. 2015). The reasons for obviously contrasting effects of p300 on stemness in breast cancer are not yet known.

p300 inhibition significantly decreased cell migration and invasion in PC3-DR cells and reduced vimentin expression. These findings indicate that p300 is involved in cellular pathways that regulate migration and invasion in PCa and are in concordance with previous studies, where they reported involvement of p300 in migration in nasopharyngeal carcinoma and breast cancer cells (Fermento et al. 2014, Liao et al. 2017).

Taken together, p300 could be a valid target in docetaxel-resistant $\mathrm{PCa}$ as it reduces the metastatic potential of docetaxel-resistant cells by reducing colony formation, migration, and invasion capability. So far, multiple factors that contribute to the development of docetaxel resistance in PCa have been described (Patterson et al. 2006, Deng et al. 2019, Kapur et al. 2019). Therefore, docetaxel resistance in $\mathrm{PCa}$ is heterogeneous, thus suggesting that medical intervention may be based on a personalized approach. This issue is particularly relevant because p300 inhibitors enter clinical trials in oncology, thus pointing to appropriate selection of patients who

This work is licensed under a Creative Commons Attribution 4.0 International License. 
will benefit from specific p300 targeting in combinations with other drugs approved or tested for treatment of PCa.

\section{Supplementary materials}

This is linked to the online version of the paper at https://doi.org/10.1530/ ERC-19-0488.

\section{Declaration of interest}

The authors declare that there is no conflict of interest that could be perceived as prejudicing the impartiality of the research reported.

\section{Funding}

This work was supported by the Austrian Science Fund FWF grant W1101-B12 to Z Culig and P31122 to N Sampson.

\section{Author contribution statement}

M Gruber and L Ferrone performed experiments. M Gruber analyzed data and wrote the first version of the manuscript. M Puhr organized and supervised immunohistochemical analyses and supervised in vitro experiments with chemoresistance models. $T$ Furlan performed experiments with the bromodomain inhibitor. F R Santer, T Furlan, I Eder, and N Sampson provided experimental suggestions and analyzed data. M Puhr and G Schäfer established TMA. F Handle established the experimental system, performed the bioinformatic analysis of the transcriptome dataset, and supervised in vitro experiments. Z Culig was responsible for conception and design of the study, data analysis, and supervision. All authors contributed to manuscript writing.

\section{Acknowledgements}

The authors thank Sarah Peer for immunohistochemical staining; Eberhard Steiner, M.Sc., for patient selection and statistical analysis; Prof Walther Parson for cell line authentication; Prof Dr. William Watson for providing CWR22RV1 and CWR22RV1-DR cells; Dr Martin Hermann for help with immunofluorescence staining; Prof Helmut Klocker and $\mathrm{Dr}$ Maximilian Brandt for proofreading the manuscript, and all members of the Division of Experimental Urology at the Medical University of Innsbruck for helpful discussions. F Handle and Z Culig, the joint senior authors.

\section{References}

Asaduzzaman M, Constantinou S, Min H, Gallon J, Lin ML, Singh P, Raguz S, Ali S, Shousha S, Coombes RC, et al. 2017 Tumour suppressor EP300, a modulator of paclitaxel resistance and stemness, is downregulated in metaplastic breast cancer. Breast Cancer Research and Treatment 163 461-474. (https://doi.org/10.1007/s10549-0174202-z)

Chan HM \& La Thangue NB 2001 p300/CBP proteins: HATs for transcriptional bridges and scaffolds. Journal of Cell Science $\mathbf{1 1 4}$ 2363-2373.

Cho MH, Park JH, Choi HJ, Park MK, Won HY, Park YJ, Lee CH, Oh SH, Song YS, Kim HS, et al. 2015 DOT1L cooperates with the c-Myc-p300 complex to epigenetically derepress CDH1 transcription factors in breast cancer progression. Nature Communications 6 7821. (https:// doi.org/10.1038/ncomms8821)
Comuzzi B, Nemes C, Schmidt S, Jasarevic Z, Lodde M, Pycha A, Bartsch G, Offner F, Culig Z \& Hobisch A 2004 The androgen receptor co-activator CBP is up-regulated following androgen withdrawal and is highly expressed in advanced prostate cancer. Journal of Pathology 204 159-166. (https://doi.org/10.1002/path.1609)

Debes JD, Schmidt LJ, Huang H \& Tindall DJ 2002 p300 mediates androgen-independent transactivation of the androgen receptor by interleukin 6. Cancer Research 62 5632-5636.

Debes JD, Sebo TJ, Lohse CM, Murphy LM, Haugen DA \& Tindall DJ 2003 p300 in prostate cancer proliferation and progression. Cancer Research 63 7638-7640.

Deng L, Gu X, Zeng T, Xu F, Dong Z, Liu C \& Chao H 2019 Identification and characterization of biomarkers and their functions for docetaxel-resistant prostate cancer cells. Oncology Letters 18 3236-3248. (https://doi.org/10.3892/ol.2019.10623)

Fermento ME, Gandini NA, Salomon DG, Ferronato MJ, Vitale CA, Arevalo J, Lopez Romero A, Nunez M, Jung M, Facchinetti MM, et al. 2014 Inhibition of p300 suppresses growth of breast cancer. Role of p300 subcellular localization. Experimental and Molecular Pathology 97 411-424. (https://doi.org/10.1016/j.yexmp.2014.09.019)

Gao XN, Lin J, Ning QY, Gao L, Yao YS, Zhou JH, Li YH, Wang LL \& Yu L 2013 A histone acetyltransferase p300 inhibitor C646 induces cell cycle arrest and apoptosis selectively in AML1-ETO-positive AML cells. PLOS ONE 8 e55481. (https://doi.org/10.1371/journal. pone.0055481)

Hanzelmann S, Castelo R \& Guinney J 2013 GSVA: gene set variation analysis for microarray and RNA-seq data. BMC Bioinformatics 147. (https://doi.org/10.1186/1471-2105-14-7)

Hatano K, Yamaguchi S, Nimura K, Murakami K, Nagahara A, Fujita K, Uemura M, Nakai Y, Tsuchiya M, Nakayama M, et al. 2013 Residual prostate cancer cells after docetaxel therapy increase the tumorigenic potential via constitutive signaling of CXCR4, ERK1/2 and c-Myc. Molecular Cancer Research 11 1088-1100. (https://doi. org/10.1158/1541-7786.MCR-13-0029-T)

Heemers HV, Sebo TJ, Debes JD, Regan KM, Raclaw KA, Murphy LM, Hobisch A, Culig Z \& Tindall DJ 2007 Androgen deprivation increases p300 expression in prostate cancer cells. Cancer Research $\mathbf{6 7}$ 3422-3430. (https://doi.org/10.1158/0008-5472.CAN-06-2836)

Iyer NG, Ozdag H \& Caldas C 2004 p300/CBP and cancer. Oncogene 23 4225-4231. (https://doi.org/10.1038/sj.onc.1207118)

James ND, Sydes MR, Clarke NW, Mason MD, Dearnaley DP, Spears MR, Ritchie AW, Parker CC, Russell JM, Attard G, et al. 2016 Addition of docetaxel, zoledronic acid, or both to first-line long-term hormone therapy in prostate cancer (STAMPEDE): survival results from an adaptive, multiarm, multistage, platform randomised controlled trial. Lancet 387 1163-1177. (https://doi.org/10.1016/S01406736(15)01037-5)

Kalkhoven E 2004 CBP and p300: HATs for different occasions. Biochemical Pharmacology 68 1145-1155. (https://doi.org/10.1016/j. bcp.2004.03.045)

Kapur N, Mir H, Sonpavde GP, Jain S, Bae S, Lillard JW. \& Singh S 2019 Prostate cancer cells hyper-activate CXCR6 signaling by cleaving CXCL16 to overcome effect of docetaxel. Cancer Letters 454 1-13. (https://doi.org/10.1016/j.canlet.2019.04.001)

Koh CM, Bieberich CJ, Dang CV, Nelson WG, Yegnasubramanian S \& De Marzo AM 2010 MYC and prostate cancer. Genes and Cancer 1 617-628. (https://doi.org/10.1177/1947601910379132)

Kumar A, Coleman I, Morrissey C, Zhang X, True LD, Gulati R, Etzioni R, Bolouri H, Montgomery B, White T, et al. 2016 Substantial interindividual and limited intraindividual genomic diversity among tumors from men with metastatic prostate cancer. Nature Medicine 22 369-378. (https://doi.org/10.1038/nm.4053)

Kyriakopoulos CE, Chen YH, Carducci MA, Liu G, Jarrard DF, Hahn NM, Shevrin DH, Dreicer R, Hussain M, Eisenberger M, et al. 2018 Chemohormonal therapy in metastatic hormone-sensitive prostate cancer: long-term survival analysis of the randomized phase III

This work is licensed under a Creative Commons Attribution 4.0 International License. 
E3805 CHAARTED trial. Journal of Clinical Oncology 36 1080-1087. (https://doi.org/10.1200/JCO.2017.75.3657)

Lasko LM, Jakob CG, Edalji RP, Qiu W, Montgomery D, Digiammarino EL, Hansen TM, Risi RM, Frey R, Manaves V, et al. 2017 Discovery of a selective catalytic p300/CBP inhibitor that targets lineage-specific tumours. Nature 550 128-132. (https://doi. org/10.1038/nature24028)

Lavaud P, Gravis G, Foulon S, Joly F, Oudard S, Priou F, Latorzeff I, Mourey L, Soulie M, Delva R, et al. 2018 Anticancer activity and tolerance of treatments received beyond progression in men treated upfront with androgen deprivation therapy with or without docetaxel for metastatic castration-naive prostate cancer in the GETUG-AFU 15 phase 3 trial. European Urology 73 696-703. (https:// doi.org/10.1016/j.eururo.2017.09.022)

Li C, Xiao XQ, Qian YH \& Zhou ZY 2019 The CtBP1-p300-FOXO3a transcriptional complex represses the expression of the apoptotic regulators Bax and Bim in human osteosarcoma cells. Journal of Cellular Physiology 234 22365-22377. (https://doi.org/10.1002/jcp.28802)

Liao ZW, Zhao L, Cai MY, Xi M, He LR, Yu F, Zhou TC \& Liu MZ 2017 P300 promotes migration, invasion and epithelial-mesenchymal transition in a nasopharyngeal carcinoma cell line. Oncology Letters 13 763-769. (https://doi.org/10.3892/ol.2016.5491)

Marin-Aguilera M, Codony-Servat J, Reig Ò, Lozano JJ, Fernandez PL, Pereira MV, Jimenez N, Donovan M, Puig P, Mengual L, et al. 2014 Epithelial-to-mesenchymal transition mediates docetaxel resistance and high risk of relapse in prostate cancer. Molecular Cancer Therapeutics 13 1270-1284. (https://doi.org/10.1158/1535-7163.MCT13-0775)

Martin SK, Banuelos CA, Sadar MD \& Kyprianou N 2015 N-terminal targeting of androgen receptor variant enhances response of castration resistant prostate cancer to taxane chemotherapy. Molecular Oncology 8 628-639. (https://doi.org/10.1016/j. molonc.2014.10.014)

Ogiwara H, Sasaki M, Mitachi T, Oike T, Higuchi S, Tominaga Y \& Kohno T 2016 Targeting p300 addiction in CBP-deficient cancers causes synthetic lethality by apoptotic cell death due to abrogation of MYC expression. Cancer Discovery 6 430-445. (https://doi. org/10.1158/2159-8290.CD-15-0754)

Patterson SG, Wei S, Chen X, Sallman DA, Gilvary DL, Zhong B, PowSang J, Yeatman T \& Djeu JY 2006 Novel role of Stat1 in the development of docetaxel resistance in prostate tumor cells. Oncogene 25 6113-6122. (https://doi.org/10.1038/sj.onc.1209632)

Puhr M, Hoefer J, Schafer G, Erb HH, Oh SJ, Klocker H, Heidegger I, Neuwirt H \& Culig Z 2012 Epithelial-to-mesenchymal transition leads to docetaxel resistance in prostate cancer and is mediated by reduced expression of miR-200c and miR-205. American Journal of
Pathology 181 2188-2201. (https://doi.org/10.1016/j. ajpath.2012.08.011)

Qin J, Lee HJ, Wu SP, Lin SC, Lanz RB, Creighton CJ, Demayo FJ, Tsai SY \& Tsai MJ 2014 Androgen deprivation-induced NCoA2 promotes metastatic and castration-resistant prostate cancer. Journal of Clinical Investigation 124 5013-5026. (https://doi.org/10.1172/JCI76412)

Ramos YF, Hestand MS, Verlaan M, Krabbendam E, Ariyurek Y, Van Galen M, Van Dam H, Van Ommen GJ, Den Dunnen JT, Zantema A, et al. 2010 Genome-wide assessment of differential roles for p300 and CBP in transcription regulation. Nucleic Acids Research $\mathbf{3 8}$ 5396-5408. (https://doi.org/10.1093/nar/gkq184)

Santer FR, Hoschele PP, Oh SJ, Erb HH, Bouchal J, Cavarretta IT, Parson W, Meyers DJ, Cole PA \& Culig Z 2011 Inhibition of the acetyltransferases p300 and CBP reveals a targetable function for p300 in the survival and invasion pathways of prostate cancer cell lines. Molecular Cancer Therapeutics 10 1644-1655. (https://doi. org/10.1158/1535-7163.MCT-11-0182)

Tang W, Zhou W, Xiang L, Wu X, Zhang P, Wang J, Liu G, Zhang W, Peng Y, Huang X, et al. 2019 The p300/YY1/miR-500a-5p/HDAC2 signalling axis regulates cell proliferation in human colorectal cancer. Nature Communications 10 663. (https://doi.org/10.1038/ s41467-018-08225-3)

Tannock IF, De Wit R, Berry WR, Horti J, Pluzanska A, Chi KN, Oudard S, Theodore C, James ND, Turesson I, et al. 2004 Docetaxel plus prednisone or mitoxantrone plus prednisone for advanced prostate cancer. New England Journal of Medicine 351 1502-1512. (https://doi.org/10.1056/NEJMoa040720)

Thadani-Mulero M, Portella L, Sun S, Sung M, Matov A, Vessella RL, Corey E, Nanus DM, Plymate SR \& Giannakakou P 2014 Androgen receptor splice variants determine taxane sensitivity in prostate cancer. Cancer Research 74 2270-2282. (https://doi.org/10.1158/00085472.CAN-13-2876)

Vazquez R, Civenni G, Kokanovic A, Shinde D, Cantergiani J, Marchetti M, Zoppi G, Ruggeri B, Liu PCC, Carbone GM, et al. 2019 Efficacy of novel bromodomain and extraterminal inhibitors in combination with chemotherapy for castration-resistant prostate cancer. European Urology Oncology [epub]. (https://doi.org/10.1016/j.euo.2019.07.013)

Wang YC, Wu YS, Hung CY, Wang SA, Young MJ, Hsu TI \& Hung JJ 2018 USP24 induces IL-6 in tumor-associated microenvironment by stabilizing p300 and beta-TrCP and promotes cancer malignancy. Nature Communications 9 3996. (https://doi.org/10.1038/s41467-01806178-1)

Zhu ML, Horbinski CM, Garzotto M, Qian DZ, Beer TM \& Kyprianou N 2010 Tubulin-targeting chemotherapy impairs androgen receptor activity in prostate cancer. Cancer Research 70 7992-8002. (https:// doi.org/10.1158/0008-5472.CAN-10-0585)

Received in final form 10 January 2020

Accepted 17 January 2020

Accepted Manuscript published online 17 January 2020 (c) 2020 The authors Published by Bioscientifica Ltd. Printed in Great Britain

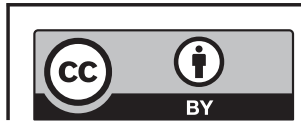

This work is licensed under a Creative Commons Attribution 4.0 International License. 